\title{
Mental burden and its risk and protective factors during the early phase of the SARS- CoV-2 pandemic: systematic review and meta-analyses
}

Angela M. Kunzler ${ }^{1,2^{*}+}$ (D) Nikolaus Röthke ${ }^{1+}$, Lukas Günthner ${ }^{2}$, Jutta Stoffers-Winterling ${ }^{1,2}$, Oliver Tüscher ${ }^{1,2}$, Michaela Coenen ${ }^{3,4}$, Eva Rehfuess ${ }^{3,4}$, Guido Schwarzer ${ }^{5}$, Harald Binder ${ }^{5}$, Christine Schmucker ${ }^{6}$, Joerg J. Meerpohl $1^{6,7}$ and Klaus Lieb ${ }^{1,2^{*}}$

\begin{abstract}
Background: Mental burden due to the SARS-CoV-2 pandemic has been widely reported for the general public and specific risk groups like healthcare workers and different patient populations. We aimed to assess its impact on mental health during the early phase by comparing pandemic with prepandemic data and to identify potential risk and protective factors.

Methods: For this systematic review and meta-analyses, we systematically searched PubMed, PsycINFO, and Web of Science from January 1, 2019 to May 29, 2020, and screened reference lists of included studies. In addition, we searched PubMed and PsycINFO for prepandemic comparative data. Survey studies assessing mental burden by the SARS-CoV-2 pandemic in the general population, healthcare workers, or any patients (eg, COVID-19 patients), with a broad range of eligible mental health outcomes, and matching studies evaluating prepandemic comparative data in the same population (if available) were included. We used multilevel meta-analyses for main, subgroup, and sensitivity analyses, focusing on (perceived) stress, symptoms of anxiety and depression, and sleep-related symptoms as primary outcomes.

(Continued on next page)
\end{abstract}

\footnotetext{
*Correspondence: angela.kunzler@lir-mainz.de; klaus.lieb@unimedizinmainz.de

Angela M. Kunzler and Nikolaus Röthke shared first authorship.

${ }^{1}$ Department of Psychiatry and Psychotherapy, University Medical Center of the Johannes Gutenberg University Mainz, Mainz, Germany

Full list of author information is available at the end of the article
}

(c) The Author(s). 2021 Open Access This article is licensed under a Creative Commons Attribution 4.0 International License, which permits use, sharing, adaptation, distribution and reproduction in any medium or format, as long as you give appropriate credit to the original author(s) and the source, provide a link to the Creative Commons licence, and indicate if changes were made. The images or other third party material in this article are included in the article's Creative Commons licence, unless indicated otherwise in a credit line to the material. If material is not included in the article's Creative Commons licence and your intended use is not permitted by statutory regulation or exceeds the permitted use, you will need to obtain permission directly from the copyright holder. To view a copy of this licence, visit http://creativecommons.org/licenses/by/4.0/ The Creative Commons Public Domain Dedication waiver (http://creativecommons.org/publicdomain/zero/1.0/) applies to the data made available in this article, unless otherwise stated in a credit line to the data. 


\begin{abstract}
(Continued from previous page)
Results: Of 2429 records retrieved, 104 were included in the review ( $n=208,261$ participants), 43 in the meta-analysis ( $n=71,613$ participants). While symptoms of anxiety (standardized mean difference [SMD] 0.40; 95\% Cl 0.15-0.65) and depression (SMD 0.67; 95\% Cl 0.07-1.27) were increased in the general population during the early phase of the pandemic compared with prepandemic conditions, mental burden was not increased in patients as well as healthcare workers, irrespective of COVID-19 patient contact. Specific outcome measures (eg, Patient Health Questionnaire) and older comparative data (published $\geq 5$ years ago) were associated with increased mental burden. Across the three population groups, existing mental disorders, female sex, and concerns about getting infected were repeatedly reported as risk factors, while older age, a good economic situation, and education were protective.
\end{abstract}

Conclusions: This meta-analysis paints a more differentiated picture of the mental health consequences in pandemic situations than previous reviews. High-quality, representative surveys, high granular longitudinal studies, and more research on protective factors are required to better understand the psychological impacts of the SARS-CoV-2 pandemic and to help design effective preventive measures and interventions that are tailored to the needs of specific population groups.

Keywords: SARS-CoV-2, COVID-19, Early phase, Psychological distress, Pandemic, Health personnel, Systematic review, Meta-analysis, Anxiety, Depression

\section{Introduction}

The emergence of novel severe acute respiratory syndrome coronavirus 2 (SARS-CoV-2) was described for the first time in Wuhan, China $[1,2]$ and declared a public health emergency of international concern on 30 January 2020 [3]. The virus spread rapidly and, as of January 14,2021 , led to $90,759,370$ confirmed infections and 1,963,169 deaths worldwide [4].

During the early phase of the pandemic, many countries adopted drastic measures, including testing, tracing, selfisolation, and quarantine measures as well as broader population measures ranging from travel bans, school closures, assembly restrictions, curfews, to full lockdowns [57]. Besides substantial stressors for individuals and the general public (eg, social isolation, reduced income, restructuring of school, university, and work life) and healthcare systems (eg, disruption of essential health services) $[8,9]$, the SARS-CoV-2 pandemic has had major socio-economic consequences for the affected countries (eg, global supply chain disruptions) $[10,11]$. By drastically changing our way of social interaction (eg, social distancing), it continues to affect many areas of daily life and in line with this social life and participation.

The disease-related threats, containment measures, and associated stressors may have a negative psychological impact on the community at large and potentially even more so on specific risk groups [12-17]. Given the work-related stressors in the context of disease outbreaks (eg, high workload, risk of infection, triage decisions), healthcare workers may suffer from a particularly high burden $[18]^{1,2}[20-22]$. Patients with pre-existing

\footnotetext{
${ }^{1}$ included pandemic studies.

${ }^{2}$ studies already considered in previous systematic review (Gilan, Röthke and colleagues) [19].
}

physical or mental conditions (eg, chronically ill individuals, psychiatric patients, geriatric patients), people with confirmed COVID-19 diagnosis, those recovering from the infection, or suffering from long COVID-19, and subgroups with special risk exposure (eg, caregivers) may also be at risk of developing stress-related mental symptoms [15, 22-28].

Various systematic reviews have synthesized the evidence on psychiatric symptoms associated with previous highly contagious infectious disease outbreaks (eg, Ebola, SARS-CoV) and the SARS-CoV-2 pandemic [20, 24, 29$35]$, some of them also narratively summarizing risk and protective factors for mental health [20, 30, 31, 33, 34]. Several meta-analyses have been conducted, either calculating the pooled prevalence of mental symptoms or odds ratios for the risk of mental burden attributable to the SARS-CoV-2 pandemic [20, 24, 29, 32, 33, 36]. Potential moderators of the negative mental health impact were also partly investigated [32]. International evidence indicates an elevated level of mental symptoms in the general public, including symptoms of anxiety, depression, and stress [30-33, 36]. Confirming the risk status of healthcare workers, several reviews also found an increased prevalence of mental symptoms in this group $[18]^{1,2}[20,29,31,32]$. Finally, a few studies in patient populations (eg, COVID-19 patients, patients with preexisting mental or physical conditions) show increased mental burden [24, 31-33].

There are several shortcomings of reviews published to date. Most either focus on the general population, healthcare workers, or patients, with only few publications examining the level of mental burden across all three specified, most relevant population groups [31-33]. Further limitations included a limited search strategy [31], language restrictions $[24,30,31,33]$, or a missing 
preregistration [20, 24, 29-31, 33, 36]. Most importantly, all but one systematic review failed to compare the mental burden during an ongoing pandemic with the burden before the pandemic [31]. Such comparisons, however, are necessary to quantify the mental burden specifically attributable to the current pandemic. We therefore aimed to assess the mental health impact of the SARS-CoV-2 pandemic by comparing data from the early phase of the current pandemic with prepandemic data in the general population, healthcare workers, and patients. We aimed to identify population-specific risk and protective factors for mental health.

\section{Methods}

\section{Review registration}

This systematic review [37] was preregistered with PROSPERO (registration no. CRD42020193249) with the title 'Psychological distress, protective factors and resilience during the SARS-CoV-2 pandemic: a systematic review and meta-analysis with comparison to standard data'. Details of the methods are presented in the Additional file 1. The MOOSE Checklist for Meta-analyses of Observational Studies and differences between the protocol and the final review are presented in eTables 1 and 2.

\section{Search strategy and selection criteria}

We searched three bibliographic databases from January 1, 2019 to May 29, 2020 (PubMed, PsycINFO, and Web of Science) and inspected the reference lists of included studies. The search strategy comprised terms associated with mental health, pandemics, and the populations of interest (see eMethods 1 in Additional file 1). There were no restrictions concerning language, publication date, or publication format. We did not consider preprint articles. If not reported within a study, we systematically searched for prepandemic comparative data in the same or a similar population (PubMed, PsycINFO; see eMethods 2).

The populations of interest comprised the general population, healthcare workers, and any patients (eg, COVID-19 patients, those with pre-existing physical or mental conditions; eTable 3). Participants were included irrespective of age, health, or employment status. We did not consider infectious disease outbreaks other than due to SARS-CoV-2. To be eligible for the review, studies had to assess at least one mental health outcome, with a broad range of eligible outcomes (ie, anxiety and worrying, depression, posttraumatic stress, sleep, stress, general psychological distress). These outcomes were also considered for a descriptive synthesis of the prevalence (see data analysis). We included original research articles reporting on cross-sectional and longitudinal surveys.
All pandemic studies meeting these criteria were included but were only taken forward to pairwise metaanalyses if using a validated outcome measure and if prepandemic comparative data were available (eTables 4, 5). These were defined as data collected before the exposure to the current pandemic, and in the absence of other disease outbreaks or macrostressors (eg, disasters), in the same country and population group (if available) and using the same outcome measure. In contrast to the review, we only focused on the four most frequently reported mental health outcomes (primary outcomes), including symptoms related to stress, anxiety, depression, or sleep. Posttraumatic stress, although reported more often than sleep, was not considered for pairwise metaanalyses. As this outcome is usually measured in the aftermath of macro-stressors, we were not able to identify adequate comparative data as mentioned above. Comparative data were selected stepwise using four levels to ensure best available comparability between SARS-CoV-2 exposure ('pandemic') studies and prepandemic ('comparative') studies. If representative studies in the same country and population (level 1) were not available, we used prepandemic studies in the same (level 2) or an alternative population (level 3 ; eg, healthcare workers compared with the general population), before resorting to the best available data in a similar country (level 4).

\section{Study selection, data extraction, and quality assessment}

The study selection process for the pandemic studies at the level of titles/abstracts and full-texts was performed in duplicate by two reviewers independently (NR, LG). Any disagreements were resolved by discussion or by consulting a third reviewer $(\mathrm{KL})$. At both title/abstract $(\kappa=0.90)$ and full-text level $(\kappa=0.97)$, excellent interrater reliability was achieved.

Relevant information for each included study was extracted in duplicate by two reviewers (NR, LG), working independently, using a customized spreadsheet (eTable 6), which was shortened for the extraction of comparative data. Discrepancies were resolved through discussion or by a third reviewer (KL).

Three independent reviewers (NR, JSW, LG) assessed the quality of included studies using the modified National Institutes of Health (NIH) Quality Assessment Tool for Observational Cohort and CrossSectional Studies [38] (eTable 7), with disagreements being resolved by discussion or a third reviewer (KL). The level of comparability between pandemic and comparative data was assessed using a self-developed tool with four levels based on the previously mentioned levels for the stepwise selection of comparative data (eTable 8). 


\section{Data analysis}

The included studies were synthesized in narrative and tabular form, with a descriptive analysis of prevalence rates for mental health symptoms (ie, proportion of participants beyond a cut-off score reported in the included study) and of risk and protective factors. If adequate comparative data for any of the primary outcomes were available, pairwise meta-analyses were performed for the general population, healthcare workers, and patients, respectively (eMethods 3). Given the multiple uses of comparative studies, we used multilevel meta-analyses [39] for the general population and healthcare workers, with pandemic studies being clustered according to prepandemic comparators. For patients, the multilevel model reduces to the classic random-effects model as different comparative studies were available. Prediction intervals were calculated in meta-analyses with at least four studies to take the large between-study heterogeneity into account [40].

Two sensitivity analyses referred to the quality of pandemic studies and the level of comparability (see Search strategy and selection criteria), by limiting the analyses to very comparable pandemic and prepandemic studies (ie, level 1 and 2 mentioned above).

Subgroup analyses for each of the three groups were performed for the surveyed populations (eg, age), characteristics of the pandemic studies (eg, survey start) and of comparative data (eg, publication year), and the relationship of sample sizes in pandemic versus comparative studies, in order to identify potential sources of heterogeneity of the psychological impact of the SARSCoV-2 pandemic.

\section{Results}

Details of the results are presented in the Additional file 2. The systematic search for studies performed during the SARS-CoV-2 pandemic identified 2429 records from database searches and 17 additional records from reference lists, of which 104 studies were included in the review and 43 studies in the metaanalyses (Fig. 1). Of the 104 eligible studies, most studies were performed in the general population (50 studies), followed by 30 studies in healthcare workers, and seven studies in various patient populations. Seventeen studies included mixed samples. Across the three population groups, a total of 208,261 participants ranging from 51 to 52,730 participants $[41,42]^{1}$ from the pandemic studies were included in the review, the number of participants considered in the meta-analyses, in total 71,613 , ranged from 127 to 60,213 participants (eTable 9).

The study characteristics of the 104 included pandemic studies (early phase) are presented in Table 1.

Although we imposed no restrictions on the age limits, we identified no studies conducted in children but did find some studies in the general population that included participants below the age of 18 years $[47,58]^{1}$.

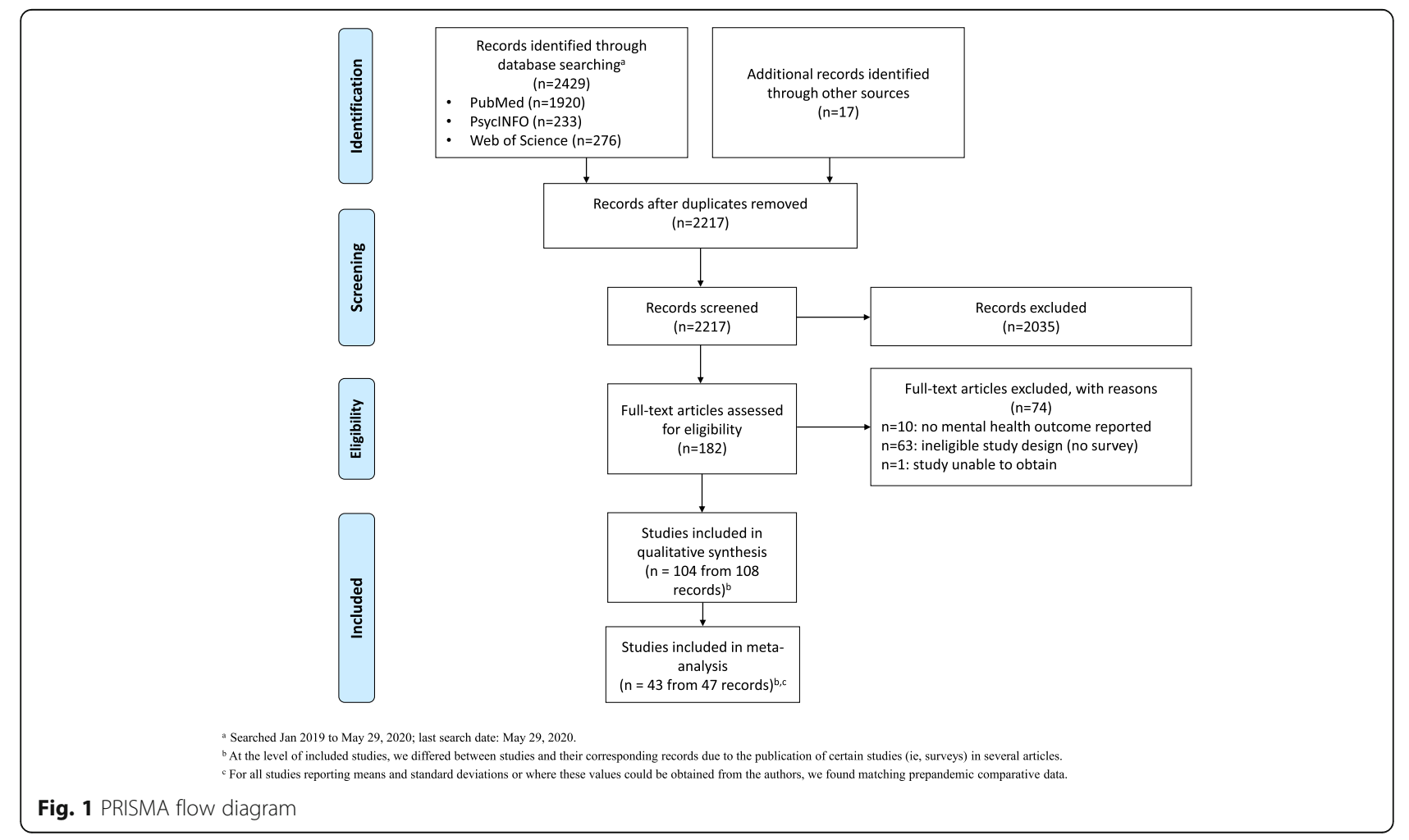


Thus, the mean age of participants in the pandemic studies ranged from 20 (SD not reported) to 56.9 (SD 7.6) years $[49,99]^{1}$. The studies covered Asia (67 studies $[26,41-43,49,50,54,57,58,60,62,64,71,74,76,80$, $81,85,86,88-91,93,98,101,102,104,109,111-116$, $118-123,126,124,128,131-133,136,137,140,141$, $143,145]^{1}[18,48,59,73,84,87,92,100,105,106,108$, $\left.117,134,138,139,142,144]^{1,2}[127]^{1,3}\right)$ thereof from China $[42,49,50,57,58,60,62,64,71,80,81,85,86$, 88-91, 93, 101, 104, 109, 115, 116, 118-120, 122-124, $126,128,131-133,136,140,141,143,145]^{1}[18,48,59$, $84,87,92,100,105,106,108,117,134,138,139,142$, $144]^{1,2}[127]^{1,3}$, Europe (24 studies) [47, 99, 44-46, 51$53,55,61,63,65,66,68,70,110,77,78,82,83,94,97$, $130,103,135]^{1}$, North America (six studies) $[56,67,79$, $107,146,147]^{1}$, or different continents (seven studies) $[69,72,75,95,96,125,129]^{1}$. For 13 studies investigating more than one population, several samples were considered $[130-133,135,140,141,143,145]^{1}[134$, $138,139,144]^{1,2}$. We identified 47 matching prepandemic comparative studies (eTable 10), including one pandemic study reporting adequate comparative data $[127]^{1,3}[148-193]^{3}$.

Prevalence rates of the six mental health symptoms, that were considered for the review, were available for a varying number of included pandemic studies (Table 2). The proportion of participants beyond a cut-off value in the included studies varied considerably (eg, anxiety in general population: $0.7-64.0 \%)$. Based on cut-off values reported in the primary studies (eTable 11), we found increased levels of mental burden during the SARSCoV-2 pandemic in the general population, healthcare workers, and patients regarding each of the symptoms observed during the current pandemic, that is, without considering the prepandemic situation.

In pairwise meta-analyses comparing pandemic (early phase) with prepandemic data for the four primary outcomes, however, we found only evidence for a small increase of anxiety (standardized mean difference [SMD] 0.40 ; $95 \%$ CI $0.15-0.65 ; p=.002$ ) and a moderate increase of depressive symptoms (SMD 0.67; 95\% CI 0.071.27; $p=.03$ ) in the general population. No evidence for a change in stress or sleep-related symptoms was identified (Table 3). For healthcare workers compared with healthcare staff before the pandemic, the meta-analyses showed no evidence of any effect on the primary outcomes (Table 3). The same was found for patients (Table 3); however, prepandemic data in patients were only available for four samples. Forest plots are presented in Figs. 2, 3, and eResults 1 in the Additional file 2.

Of the 104 studies, 38 studies were judged to be of fair quality and 57 studies of poor quality, with main

${ }^{3}$ prepandemic comparative studies. concerns regarding selection bias, the validity of outcome measures, and the description of the sample and the survey period (eTable 12). From nine high-quality studies, four were representative surveys [44, 47, 77, $88]^{1}$. From the 85 pairwise comparisons relevant for meta-analyses, 52 comparisons were of level- 1 and 33 of level-2 quality (eTable 13). When excluding low-quality pandemic studies (Table 3), the effects on anxiety and depressive symptoms in the general population increased. The effect on anxiety in the general population was stable in the sensitivity analysis when only best comparable data sets (ie, level-1 and level-2 comparability) were included, while there was no longer evidence for an effect on depressive symptoms (Table 3 and eResults 2 in Additional file 2).

Heterogeneity was considerable in main and sensitivity analyses, with $\mathrm{I}^{2}$ scores mostly ranging from 90 to $100 \%$ and wide prediction intervals (Table 3 ). We therefore performed subgroup analyses with at least $\mathrm{k}=5$ studies in the main analyses in attempts to explain this heterogeneity (Table 4; eResults 3 in Additional file 2).

Regarding population characteristics (pandemic studies), age was no consistent risk or protective factor. Within the general population, we identified no evidence for a subgroup difference according to stressor exposure except for elevated sleep symptoms in isolated individuals $[62]^{1}$. In healthcare workers, there was no evidence for a moderating effect of COVID-19 patient contact on mental health. In different groups of patients, we identified no evidence of differences in anxiety or depression. Compared with COVID-19 patients [131] $]^{1}$, psychiatric patients reported more stress, with the caveat of few studies $[42,132,135]^{1}$.

Among general characteristics of the pandemic studies, we found no (consistent) evidence of differences depending on when the surveys started, whether they were conducted in China, or the sample size. We found evidence of an elevated level of depressive symptoms in the general population and patients depending on the specific outcome measure employed (eg, Patient Health Questionnaire [PHQ], Zung Self-Rating Depression Scale [SDS]).

In subgroup analyses for comparative study characteristics, there was no evidence of a consistent moderation of comparison sample sizes.

Across the three populations, we identified a higher level of anxiety and depressive symptoms if included studies were compared to prepandemic data published five or more years before versus a smaller burden in comparison to prepandemic data of less than 2 years ago.

The relationship of sample sizes explained the heterogeneity of the psychological impact of the SARS-CoV-2 pandemic in the general population and patients, with evidence for elevated symptoms of anxiety if similar sample sizes were compared. 
Table 1 Study characteristics of included main studies

\begin{tabular}{|c|c|c|c|c|c|c|c|}
\hline Study & $\begin{array}{l}\text { Study } \\
\text { design }\end{array}$ & Country & $\begin{array}{l}\text { Sample size; female: No. } \\
\text { (\%); age: mean (SD) or } \\
\text { alternative information on } \\
\text { age (eg, mode) }\end{array}$ & Subgroups & $\begin{array}{l}\text { Survey } \\
\text { period }\end{array}$ & $\begin{array}{l}\text { Assessed } \\
\text { Outcomes }\end{array}$ & $\begin{array}{l}\text { Instruments } \\
\text { or scales }\end{array}$ \\
\hline \multicolumn{8}{|c|}{ General Population } \\
\hline $\begin{array}{l}\text { Ahmad et al. } \\
(2020)[43]^{1}\end{array}$ & $\mathrm{CS}, \mathrm{OBS}$ & $\begin{array}{l}\text { Iraq } \\
\text { (Kurdistan) }\end{array}$ & 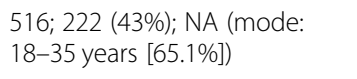 & NA & NA & $\begin{array}{l}\text { Anxiety and } \\
\text { fear }\end{array}$ & $\begin{array}{l}\text { Binary single } \\
\text { item }^{\mathrm{a}}\end{array}$ \\
\hline \multirow[t]{2}{*}{$\begin{array}{l}\text { Bacon et al. } \\
(2020)[44]^{1}\end{array}$} & $\mathrm{CS}, \mathrm{OBS}$ & $\begin{array}{l}\text { United } \\
\text { Kingdom }\end{array}$ & $\begin{array}{l}\text { 202; } 127(62.9 \%), 1 \text { diverse; } \\
33.79(12.48)\end{array}$ & NA & $\begin{array}{l}\text { March 18- } \\
19,2020\end{array}$ & $\begin{array}{l}\text { Anxiety and } \\
\text { fear }\end{array}$ & GAD-7 \\
\hline & & & & & & $\begin{array}{l}\text { Depressive } \\
\text { symptoms }\end{array}$ & BDI-II \\
\hline \multirow{3}{*}{$\begin{array}{l}\text { Bäuerle et al. } \\
\text { (2020) [45] } \\
\text { Teufel et al. } \\
(2020)[46]^{1}\end{array}$} & $\mathrm{CS}, \mathrm{OBS}$ & Germany & $\begin{array}{l}\text { 15,037; 10,633 }(70.7 \%), N A \\
\text { (mode: } 25-34 \text { years [24.8\%]) }\end{array}$ & NA & $\begin{array}{l}\text { March 10- } \\
\text { May 5, } 2020\end{array}$ & $\begin{array}{l}\text { Anxiety and } \\
\text { fear }\end{array}$ & $\begin{array}{l}\text { GAD-7, single } \\
\text { item 7-P LS }\end{array}$ \\
\hline & & & & & & $\begin{array}{l}\text { Depressive } \\
\text { symptoms }\end{array}$ & PHQ-2 \\
\hline & & & & & & $\begin{array}{l}\text { Psychological } \\
\text { Distress }\end{array}$ & DT \\
\hline $\begin{array}{l}\text { Buzzi et al. } \\
(2020)[47]^{1}\end{array}$ & $\mathrm{CS}, \mathrm{OBS}$ & Italy & 2064; NA; NA & $100 \%$ adolescents & March 2020 & $\begin{array}{l}\text { Anxiety and } \\
\text { fear }\end{array}$ & $4-P L^{a}$ \\
\hline $\begin{array}{l}\text { Cao et al. } \\
\text { (2020) [48] }\end{array}$ & $\mathrm{CS}, \mathrm{OBS}$ & China & 7143; 4975 (69.7\%); NA & NA & NA & $\begin{array}{l}\text { Anxiety and } \\
\text { fear }\end{array}$ & GAD-7 \\
\hline \multirow[t]{2}{*}{$\begin{array}{l}\text { Chang et al. } \\
(2020)[49]^{1}\end{array}$} & \multirow[t]{2}{*}{ CS, OBS } & \multirow[t]{2}{*}{ China } & \multirow[t]{2}{*}{$\begin{array}{l}3881 ; 2447(63.1 \%) ; 20.00(\mathrm{NA}) ; \\
\left.P_{25}=19.00, P_{75}=22.00\right]\end{array}$} & \multirow{2}{*}{$\begin{array}{l}100 \% \text { students }^{\mathrm{b}} \\
\text { medical students } \\
(n=3359)\end{array}$} & \multirow{2}{*}{$\begin{array}{l}\text { January 31, } \\
2019- \\
\text { February 3, } \\
2020\end{array}$} & $\begin{array}{l}\text { Anxiety and } \\
\text { fear }\end{array}$ & GAD-7 \\
\hline & & & & & & $\begin{array}{l}\text { Depressive } \\
\text { symptoms }\end{array}$ & PHQ-9 \\
\hline \multirow[t]{2}{*}{$\begin{array}{l}\text { Gao J et al. } \\
(2020)[50]^{i}\end{array}$} & \multirow[t]{2}{*}{$\mathrm{CS}, \mathrm{OBS}$} & \multirow[t]{2}{*}{ China } & \multirow[t]{2}{*}{$4872 ; 3267$ (67.7\%); 32.3 (10.0) } & \multirow[t]{2}{*}{ NA } & \multirow{2}{*}{$\begin{array}{l}\text { January } 31- \\
\text { February 02, } \\
2020\end{array}$} & $\begin{array}{l}\text { Anxiety and } \\
\text { fear }\end{array}$ & GAD-7 \\
\hline & & & & & & $\begin{array}{l}\text { Depressive } \\
\text { symptoms }\end{array}$ & WHO- $5^{c}$ \\
\hline \multirow{3}{*}{$\begin{array}{l}\text { Germani } \\
\text { et al. (2020) } \\
{[51]^{1}}\end{array}$} & \multirow[t]{3}{*}{$\mathrm{CS}, \mathrm{OBS}$} & \multirow[t]{3}{*}{ Italy } & \multirow[t]{3}{*}{ 1011; 720 (71.2\%); 24.2 (3.6) } & \multirow[t]{3}{*}{$\begin{array}{l}100 \% \text { age between } \\
18 \text { and } 29 \text { years }\end{array}$} & \multirow[t]{3}{*}{$\begin{array}{l}\text { March 17- } \\
24,2020\end{array}$} & $\begin{array}{l}\text { Anxiety and } \\
\text { fear }\end{array}$ & STAI-Y \\
\hline & & & & & & Stress & PSS \\
\hline & & & & & & $\begin{array}{l}\text { Other } \\
\text { Outcomes }\end{array}$ & SDQ \\
\hline \multirow{4}{*}{$\begin{array}{l}\text { González- } \\
\text { Sanguino } \\
\text { et al. (2020) } \\
{[52]^{1}}\end{array}$} & \multirow[t]{4}{*}{$\mathrm{CS}, \mathrm{OBS}$} & \multirow[t]{4}{*}{ Spain } & \multirow[t]{4}{*}{ 3480; 2610 (75\%); 37-92 (NA) } & \multirow[t]{4}{*}{ NA } & \multirow[t]{4}{*}{$\begin{array}{l}\text { March 21- } \\
28,2020\end{array}$} & $\begin{array}{l}\text { Anxiety and } \\
\text { fear }\end{array}$ & GAD-2 \\
\hline & & & & & & $\begin{array}{l}\text { Depressive } \\
\text { symptoms }\end{array}$ & PHQ-2 \\
\hline & & & & & & PTSS & PCL-C-2 \\
\hline & & & & & & $\begin{array}{l}\text { Other } \\
\text { outcomes }\end{array}$ & $\begin{array}{l}\text { FACIT-Sp12, } \\
\text { MSPSS, SCS }\end{array}$ \\
\hline \multirow[t]{3}{*}{$\begin{array}{l}\text { Harper et al. } \\
(2020)[53]^{1}\end{array}$} & \multirow[t]{3}{*}{$\mathrm{CS}, \mathrm{OBS}$} & \multirow[t]{3}{*}{ UK } & \multirow[t]{3}{*}{$324 ; 162$ (50\%); 34-32 (11.71) } & \multirow[t]{3}{*}{ NA } & $\begin{array}{l}\text { March 27- } \\
28,2020\end{array}$ & $\begin{array}{l}\text { Anxiety and } \\
\text { fear }\end{array}$ & $\begin{array}{l}\text { FCV-19S, } \\
\text { PROMIS-SF } \\
\text { Anxiety }\end{array}$ \\
\hline & & & & & & $\begin{array}{l}\text { Depressive } \\
\text { symptoms }\end{array}$ & $\begin{array}{l}\text { PROMIS-SF } \\
\text { Depression }\end{array}$ \\
\hline & & & & & & $\begin{array}{l}\text { Other } \\
\text { outcomes }\end{array}$ & WHOQOL-BREF \\
\hline $\begin{array}{l}\text { Jahanshahi } \\
\text { et al. (2020) } \\
{[54]^{1}}\end{array}$ & $\mathrm{CS}, \mathrm{OBS}$ & Iran & $\begin{array}{l}\text { 1058; } 569(53-8 \%) ; \text { NA (mode: } \\
26-35 \text { years) }\end{array}$ & NA & $\begin{array}{l}\text { March 25- } \\
28,2020\end{array}$ & $\begin{array}{l}\text { Psychological } \\
\text { distress }\end{array}$ & CPDI \\
\hline $\begin{array}{l}\text { Lauri Korajlija } \\
\text { et al. (2020) } \\
{[55]^{1}}\end{array}$ & $\begin{array}{l}\text { CS } \\
\text { (repeated), } \\
\text { OBS }\end{array}$ & Croatia & $\begin{array}{l}\text { sample 1: 888; } 738^{\mathrm{d}}(83-1 \%) \\
31.3(10.45) \\
\text { sample 2: } 966 ; 732^{\mathrm{d}}(75.8 \%) \\
40(11.94)\end{array}$ & NA & $\begin{array}{l}\text { 1st period: } \\
\text { February } \\
\text { 24-NA } \\
\text { 2nd period: } \\
\text { March 19- }\end{array}$ & $\begin{array}{l}\text { Anxiety and } \\
\text { fear }\end{array}$ & $\begin{array}{l}\text { 11-items 5-P LS } \\
\text { (based on } \\
\text { Swine Flu Anx- } \\
\text { iety Items, } \\
\text { Wheaton et al. }\end{array}$ \\
\hline
\end{tabular}


Table 1 Study characteristics of included main studies (Continued)

\begin{tabular}{|c|c|c|c|c|c|c|c|}
\hline Study & $\begin{array}{l}\text { Study } \\
\text { design }\end{array}$ & Country & $\begin{array}{l}\text { Sample size; female: No. } \\
\text { (\%); age: mean (SD) or } \\
\text { alternative information on } \\
\text { age (eg, mode) }\end{array}$ & Subgroups & $\begin{array}{l}\text { Survey } \\
\text { period }\end{array}$ & $\begin{array}{l}\text { Assessed } \\
\text { Outcomes }\end{array}$ & $\begin{array}{l}\text { Instruments } \\
\text { or scales }\end{array}$ \\
\hline & & & & & NA & & $2012)^{a}$ \\
\hline \multirow[t]{2}{*}{$\begin{array}{l}\text { Lee SA et al. } \\
(2020)[56]^{1}\end{array}$} & \multirow[t]{2}{*}{$\mathrm{CS}, \mathrm{OBS}$} & \multirow[t]{2}{*}{ USA } & \multirow[t]{2}{*}{ 398; 191 (49\%); 35.91 (11.73) } & \multirow[t]{2}{*}{ NA } & \multirow[t]{2}{*}{$\begin{array}{l}\text { March 23- } \\
24,2020\end{array}$} & $\begin{array}{l}\text { Anxiety and } \\
\text { fear }\end{array}$ & $\begin{array}{l}2 \text { single items } \\
5-P L^{a}\end{array}$ \\
\hline & & & & & & $\begin{array}{l}\text { Other } \\
\text { outcomes }\end{array}$ & $\begin{array}{l}\text { Passive suicidal } \\
\text { ideation (single } \\
\text { item 5-P LS) }\end{array}$ \\
\hline \multirow[t]{2}{*}{$\begin{array}{l}\text { Lei et al. } \\
(2020)[57]^{1}\end{array}$} & \multirow[t]{2}{*}{$\mathrm{CS}, \mathrm{OBS}$} & \multirow[t]{2}{*}{ China } & \multirow[t]{2}{*}{ 1593; 976 (61.3\%); 32.3 (9.8) } & \multirow{2}{*}{$\begin{array}{l}\text { 'affected group': } \\
\text { quarantined / } \\
\text { relatives quarantined } \\
(n=420)^{b}\end{array}$} & \multirow[t]{2}{*}{$\begin{array}{l}\text { February } \\
04-10,2020\end{array}$} & $\begin{array}{l}\text { Anxiety and } \\
\text { fear }\end{array}$ & SAS \\
\hline & & & & & & $\begin{array}{l}\text { Depressive } \\
\text { symptoms }\end{array}$ & SDS \\
\hline \multirow{2}{*}{$\begin{array}{l}\text { Li Y et al. } \\
(2020)[58]^{1}\end{array}$} & \multirow{2}{*}{$\begin{array}{l}\text { CS (part of } \\
\text { longitudinal } \\
\text { cohort } \\
\text { study), OBS }\end{array}$} & \multirow[t]{2}{*}{ China } & \multirow{2}{*}{$\begin{array}{l}1442 ; 891^{d}(61.8 \%) ; N A(K-6< \\
5: 20.0[1.5] ; K-6 \geq 5: 20.0[1.6])\end{array}$} & \multirow{2}{*}{$\begin{array}{l}\text { medical students } \\
(n=764), \text { nursing } \\
\text { students ( } n=211) \text {, } \\
\text { medical technology } \\
\text { students ( } n=467)\end{array}$} & \multirow{2}{*}{$\begin{array}{l}\text { February } 7- \\
13,2020\end{array}$} & PTSS & IES-R \\
\hline & & & & & & $\begin{array}{l}\text { Psychological } \\
\text { distress }\end{array}$ & $\mathrm{K}-6$ \\
\hline $\begin{array}{l}\text { Liu N et al. } \\
(2020)[59]^{1,2}\end{array}$ & $\mathrm{CS}, \mathrm{OBS}$ & China & $\begin{array}{l}\text { 285; } 155(54.4 \%) ; \mathrm{NA}(47.7 \%< \\
35)\end{array}$ & NA & $\begin{array}{l}\text { January } 30- \\
\text { February 08, } \\
2020\end{array}$ & PTSS & PCL-5 \\
\hline \multirow[t]{2}{*}{$\begin{array}{l}\text { Liu S et al. } \\
(2020)[60]^{1}\end{array}$} & \multirow[t]{2}{*}{$\mathrm{CS}, \mathrm{OBS}$} & \multirow[t]{2}{*}{ China } & \multirow{2}{*}{$\begin{array}{l}\text { primary school: 209; } 116 \\
\left(56^{d}{ }^{d}\right) ; \text { NA } \\
\text { college: } 198 ; 130 \text { (62\%); NA }\end{array}$} & \multirow{2}{*}{$\begin{array}{l}\text { primary school } \\
\text { students, college } \\
\text { students }\end{array}$} & \multirow[t]{2}{*}{$\begin{array}{l}\text { February- } \\
\text { March, } 2020\end{array}$} & $\begin{array}{l}\text { Anxiety and } \\
\text { fear }\end{array}$ & $\begin{array}{l}3 \text { items, 4-P } \\
L^{a}\end{array}$ \\
\hline & & & & & & $\begin{array}{l}\text { Other } \\
\text { outcomes }\end{array}$ & SSS \\
\hline \multirow[t]{2}{*}{$\begin{array}{l}\text { Lopez et al. } \\
(2020)[61]^{1}\end{array}$} & \multirow[t]{2}{*}{$\mathrm{CS}, \mathrm{OBS}$} & \multirow[t]{2}{*}{ Spain } & \multirow{2}{*}{$\begin{array}{l}878 ; 544^{\mathrm{d}}(62 \%) \text { or } 636\left(72 \%{ }^{\mathrm{d}}\right) \text {, } \\
\text { data in text and Table } 1 \\
\text { inconsistent; NA (mode: } 60- \\
70 \text { years }\left[71 \%^{\mathrm{d}}\right] \text { ) }\end{array}$} & \multirow{2}{*}{$\begin{array}{l}\text { 100\% community- } \\
\text { dwelling older adults; } \\
\text { age 60-70 (n=626); } \\
\text { age } 71-80(n=252)\end{array}$} & \multirow[t]{2}{*}{ NA } & $\begin{array}{l}\text { Anxiety and } \\
\text { fear }\end{array}$ & a \\
\hline & & & & & & $\begin{array}{l}\text { Other } \\
\text { outcomes }\end{array}$ & $\begin{array}{l}\text { BRCS, Ryff's } \\
\text { PWB (subscales } \\
\text { for personal } \\
\text { growth and } \\
\text { purpose in life) }\end{array}$ \\
\hline \multirow[t]{5}{*}{$\begin{array}{l}\text { Ma et al. } \\
(2020)[62]^{1}\end{array}$} & \multirow[t]{5}{*}{$\mathrm{CS}, \mathrm{OBS}$} & \multirow[t]{5}{*}{ China } & $123 ; 71^{d}\left(57.7 \%^{d}\right) ; 37.4(10.6)$ & $\begin{array}{l}100 \% \text { isolated } \\
\text { people }^{\mathrm{b}}\end{array}$ & $\begin{array}{l}\text { January } \\
2020\end{array}$ & $\begin{array}{l}\text { Anxiety and } \\
\text { fear }\end{array}$ & $\begin{array}{l}\text { DASS-21 } \\
\text { Anxiety }\end{array}$ \\
\hline & & & & & & $\begin{array}{l}\text { Depressive } \\
\text { symptoms }\end{array}$ & $\begin{array}{l}\text { DASS-21 } \\
\text { Depression }\end{array}$ \\
\hline & & & & & & Stress & DASS-21 Stress \\
\hline & & & & & & $\begin{array}{l}\text { Sleep-related } \\
\text { symptoms }\end{array}$ & PSQI \\
\hline & & & & & & $\begin{array}{l}\text { Other } \\
\text { outcomes }\end{array}$ & SF-36 \\
\hline $\begin{array}{l}\text { Mazza et al. } \\
\text { (2020) [63] }\end{array}$ & $\mathrm{CS}, \mathrm{OBS}$ & Italy & $\begin{array}{l}\text { 2766; } 1982 \text { (71.7\%); } 32.94 \\
(13.2)\end{array}$ & NA & $\begin{array}{l}\text { March 18- } \\
22,2020\end{array}$ & $\begin{array}{l}\text { Anxiety and } \\
\text { fear }\end{array}$ & $\begin{array}{l}\text { DASS-21 } \\
\text { Anxiety }\end{array}$ \\
\hline & & & & & & $\begin{array}{l}\text { Depressive } \\
\text { symptoms }\end{array}$ & $\begin{array}{l}\text { DASS-21 } \\
\text { Depression }\end{array}$ \\
\hline & & & & & & Stress & DASS-21 Stress \\
\hline $\begin{array}{l}\text { McKay et al. } \\
(2020)[64]^{1}\end{array}$ & $\mathrm{CS}, \mathrm{OBS}$ & China & $908 ; 752$ (82.8\%); 40.37 (9.27) & NA & $\begin{array}{l}\text { February } \\
\text { 24-March } \\
15,2020\end{array}$ & $\begin{array}{l}\text { Anxiety and } \\
\text { fear }\end{array}$ & $\begin{array}{l}\text { CoVGAD-7, } \\
\text { DASS-21 } \\
\text { Anxiety }\end{array}$ \\
\hline & & & & & & $\begin{array}{l}\text { Depressive } \\
\text { symptoms }\end{array}$ & $\begin{array}{l}\text { DASS-21 } \\
\text { Depression }\end{array}$ \\
\hline $\begin{array}{l}\text { Moccia et al. } \\
(2020)[65]^{1}\end{array}$ & CS, OBS & Italy & $\begin{array}{l}\text { 500; } 298(59.6) ; \text { NA (mode: } \\
28-37 \text { years, } n=129 \text { ) }\end{array}$ & NA & $\begin{array}{l}\text { April 10-13, } \\
2020\end{array}$ & $\begin{array}{l}\text { Psychological } \\
\text { distress }\end{array}$ & $\mathrm{K}-10$ \\
\hline & & & & & & $\begin{array}{l}\text { Other } \\
\text { outcomes }\end{array}$ & TEMPS-A \\
\hline
\end{tabular}


Table 1 Study characteristics of included main studies (Continued)

\begin{tabular}{|c|c|c|c|c|c|c|c|}
\hline Study & $\begin{array}{l}\text { Study } \\
\text { design }\end{array}$ & Country & $\begin{array}{l}\text { Sample size; female: No. } \\
\text { (\%); age: mean (SD) or } \\
\text { alternative information on } \\
\text { age (eg, mode) }\end{array}$ & Subgroups & $\begin{array}{l}\text { Survey } \\
\text { period }\end{array}$ & $\begin{array}{l}\text { Assessed } \\
\text { Outcomes }\end{array}$ & $\begin{array}{l}\text { Instruments } \\
\text { or scales }\end{array}$ \\
\hline \multirow{4}{*}{$\begin{array}{l}\text { Odriozola- } \\
\text { González } \\
\text { et al. (2020) } \\
{[66]^{1}}\end{array}$} & \multirow[t]{4}{*}{ CS, OBS } & \multirow[t]{4}{*}{ Spain } & \multirow[t]{4}{*}{$2530 ; 1672$ (66.1\%); 27.9 (12.4) } & \multirow{4}{*}{$\begin{array}{l}\text { students ( } n=1944) ; \\
\text { administrative staff } \\
(n=247) ; \text { faculty } \\
\text { members and } \\
\text { academic staff ( } n= \\
339)^{\mathrm{b}}\end{array}$} & \multirow{4}{*}{$\begin{array}{l}\text { March 28- } \\
\text { April 3, } \\
2020\end{array}$} & $\begin{array}{l}\text { Anxiety and } \\
\text { fear }\end{array}$ & $\begin{array}{l}\text { DASS-21 } \\
\text { Anxiety }\end{array}$ \\
\hline & & & & & & $\begin{array}{l}\text { Depressive } \\
\text { symptoms }\end{array}$ & $\begin{array}{l}\text { DASS-21 } \\
\text { Depression }\end{array}$ \\
\hline & & & & & & Stress & DASS-21 Stress \\
\hline & & & & & & PTSS & IES \\
\hline \multirow[t]{2}{*}{$\begin{array}{l}\text { Olagoke et al. } \\
(2020)[146]^{1}\end{array}$} & \multirow[t]{2}{*}{ CS, OBS } & \multirow[t]{2}{*}{ USA } & \multirow[t]{2}{*}{$\begin{array}{l}501 ; 277(55.29 \%) ; 32.44 \\
(11.94)\end{array}$} & \multirow[t]{2}{*}{ NA } & \multirow[t]{2}{*}{$\begin{array}{l}\text { March 25, } \\
\text { 2020-NA }\end{array}$} & $\begin{array}{l}\text { Depressive } \\
\text { symptoms }\end{array}$ & PHQ-2 \\
\hline & & & & & & $\begin{array}{l}\text { Other } \\
\text { outcomes }\end{array}$ & $\begin{array}{l}\text { Perceived self- } \\
\text { efficacy (Ajzen } \\
\text { 2002) }\end{array}$ \\
\hline \multirow{3}{*}{$\begin{array}{l}\text { Ozamiz- } \\
\text { Etxebarria } \\
\text { et al. (2020) } \\
{[68]^{1}}\end{array}$} & \multirow[t]{3}{*}{$\mathrm{CS}, \mathrm{OBS}$} & \multirow[t]{3}{*}{ Spain } & \multirow[t]{3}{*}{ 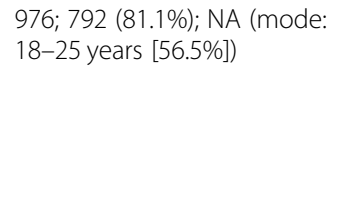 } & \multirow[t]{3}{*}{ NA } & \multirow[t]{3}{*}{$\begin{array}{l}\text { March 11- } \\
15,2020\end{array}$} & $\begin{array}{l}\text { Anxiety and } \\
\text { fear }\end{array}$ & $\begin{array}{l}\text { DASS-21 } \\
\text { Anxiety }\end{array}$ \\
\hline & & & & & & $\begin{array}{l}\text { Depressive } \\
\text { symptoms }\end{array}$ & $\begin{array}{l}\text { DASS-21 } \\
\text { Depression }\end{array}$ \\
\hline & & & & & & Stress & DASS-21 Stress \\
\hline \multirow[t]{2}{*}{$\begin{array}{l}\text { Özdin et al. } \\
(2020)[69]^{1}\end{array}$} & \multirow[t]{2}{*}{$\mathrm{CS}, \mathrm{OBS}$} & \multirow[t]{2}{*}{ Turkey } & \multirow[t]{2}{*}{$343 ; 169$ (49.2\%); $37.2(10.3)$} & \multirow[t]{2}{*}{ NA } & \multirow[t]{2}{*}{$\begin{array}{l}\text { April 14-16, } \\
2020\end{array}$} & $\begin{array}{l}\text { Anxiety and } \\
\text { fear }\end{array}$ & $\mathrm{HAl}$ \\
\hline & & & & & & $\begin{array}{l}\text { Depressive } \\
\text { symptoms }\end{array}$ & HADS \\
\hline $\begin{array}{l}\text { Perez- } \\
\text { Fuentes et al. } \\
(2020)[70]^{1}\end{array}$ & $\mathrm{CS}, \mathrm{OBS}$ & Spain & $\begin{array}{l}1014 ; 681(67.2 \%) ; 40.87 \\
(12.42)\end{array}$ & NA & $\begin{array}{l}\text { March 18- } \\
23,2020\end{array}$ & $\begin{array}{l}\text { Depressive } \\
\text { symptoms }\end{array}$ & BIP-Q5 \\
\hline $\begin{array}{l}\text { Qiu et al. } \\
\text { (2020) [41] }\end{array}$ & $\mathrm{CS}, \mathrm{OBS}$ & $\begin{array}{l}\text { China, Hong } \\
\text { Kong, Macao, } \\
\text { Taiwan }\end{array}$ & 52,$730 ; 34,131(64.7 \%)$ & NA & $\begin{array}{l}\text { January } 31- \\
\text { February 2, } \\
2020\end{array}$ & $\begin{array}{l}\text { Psychological } \\
\text { distress }\end{array}$ & CPDI \\
\hline \multirow[t]{6}{*}{$\begin{array}{l}\text { Ren et al. } \\
(2020)[71]^{1}\end{array}$} & \multirow[t]{6}{*}{$\mathrm{CS}, \mathrm{OBS}$} & \multirow[t]{6}{*}{ China } & \multirow[t]{6}{*}{$1172 ; N A ; N A$} & \multirow[t]{6}{*}{ NA } & \multirow{6}{*}{$\begin{array}{l}\text { February } \\
\text { 14-March } \\
29,2020\end{array}$} & $\begin{array}{l}\text { Anxiety and } \\
\text { fear }\end{array}$ & GAD-7 \\
\hline & & & & & & $\begin{array}{l}\text { Depressive } \\
\text { symptoms }\end{array}$ & PHQ-9 \\
\hline & & & & & & Stress & PSS-10 \\
\hline & & & & & & $\begin{array}{l}\text { Sleep-related } \\
\text { symptoms }\end{array}$ & $|S|$ \\
\hline & & & & & & PTSS & PCL-5 \\
\hline & & & & & & $\begin{array}{l}\text { Other } \\
\text { outcomes }\end{array}$ & $\begin{array}{l}\text { MINI suicidality } \\
\text { module }\end{array}$ \\
\hline $\begin{array}{l}\text { Reznik et al. } \\
(2020)[72]^{1}\end{array}$ & $\mathrm{CS}, \mathrm{OBS}$ & $\begin{array}{l}\text { Russia \& } \\
\text { Belarus }\end{array}$ & $850 ; 622(73.2 \%) ; 34.8(13.0)$ & NA & $\begin{array}{l}\text { after March } \\
27,2020\end{array}$ & $\begin{array}{l}\text { Anxiety and } \\
\text { fear }\end{array}$ & FCV-19S \\
\hline $\begin{array}{l}\text { Roy et al. } \\
(2020)[73]^{1,2}\end{array}$ & $\mathrm{CS}, \mathrm{OBS}$ & India & $662 ; 339$ (51.2\%); 29.09 (8.83) & NA & $\begin{array}{l}\text { March 22- } \\
24,2020\end{array}$ & $\begin{array}{l}\text { Anxiety and } \\
\text { fear }\end{array}$ & $\begin{array}{l}18 \text { items 5-P } \\
\mathrm{LS}^{\mathrm{a}}\end{array}$ \\
\hline \multirow[t]{2}{*}{$\begin{array}{l}\text { Sakib et al. } \\
(2020)[74]^{1}\end{array}$} & $\mathrm{CS}, \mathrm{OBS}$ & Bangladesh & $8550 ; 3760$ (44\%); 26.5 (9.1) & NA & $\begin{array}{l}\text { April 1-10, } \\
2020\end{array}$ & $\begin{array}{l}\text { Anxiety and } \\
\text { fear }\end{array}$ & FCV-19S \\
\hline & & & & & & $\begin{array}{l}\text { Depressive } \\
\text { symptoms }\end{array}$ & PHQ-9 \\
\hline $\begin{array}{l}\text { Satici et al. } \\
(2020)[75]^{1}\end{array}$ & $\mathrm{CS}, \mathrm{OBS}$ & Turkey & 1304; 917 (70.3\%); 29.5 (10.5) & NA & NA & $\begin{array}{l}\text { Anxiety and } \\
\text { fear }\end{array}$ & $\begin{array}{l}\text { DASS-21 } \\
\text { Anxiety, FCV- } \\
195\end{array}$ \\
\hline & & & & & & $\begin{array}{l}\text { Depressive } \\
\text { symptoms }\end{array}$ & $\begin{array}{l}\text { DASS-21 } \\
\text { Depression }\end{array}$ \\
\hline & & & & & & Stress & DASS-21 Stress \\
\hline Shammi et al. & $\mathrm{CS}, \mathrm{OBS}$ & Bangladesh & $1066 ; 405$ (38.5\%); 27.80 & NA & March 28- & Psychological & COVID-19 \\
\hline
\end{tabular}


Table 1 Study characteristics of included main studies (Continued)

\begin{tabular}{|c|c|c|c|c|c|c|c|}
\hline Study & $\begin{array}{l}\text { Study } \\
\text { design }\end{array}$ & Country & $\begin{array}{l}\text { Sample size; female: No. } \\
\text { (\%); age: mean (SD) or } \\
\text { alternative information on } \\
\text { age (eg, mode) }\end{array}$ & Subgroups & $\begin{array}{l}\text { Survey } \\
\text { period }\end{array}$ & $\begin{array}{l}\text { Assessed } \\
\text { Outcomes }\end{array}$ & $\begin{array}{l}\text { Instruments } \\
\text { or scales }\end{array}$ \\
\hline$(2020)[76]^{1}$ & & & $(10.05)$ & & 30,2020 & distress & $\begin{array}{l}\text { related mental } \\
\text { distress }(5 \\
\text { items 5-P LS) }\end{array}$ \\
\hline \multirow[t]{2}{*}{$\begin{array}{l}\text { Shevlin et al. } \\
(2020)[77]^{1}\end{array}$} & \multirow[t]{2}{*}{$\mathrm{CS}, \mathrm{OBS}$} & \multirow[t]{2}{*}{ UK } & \multirow[t]{2}{*}{ 2025; 1047 (51.9\%); 45.4 (15.9) } & \multirow[t]{2}{*}{ NA } & \multirow[t]{2}{*}{$\begin{array}{l}\text { March 23- } \\
28,2020\end{array}$} & $\begin{array}{l}\text { Anxiety and } \\
\text { fear }\end{array}$ & $\begin{array}{l}\text { GAD-7, VAS on } \\
\text { COVID-19 } \\
\text { anxiety }\end{array}$ \\
\hline & & & & & & $\begin{array}{l}\text { Other } \\
\text { outcomes }\end{array}$ & PHQ-15 \\
\hline $\begin{array}{l}\text { Soraci et al. } \\
(2020)[78]^{1}\end{array}$ & $\mathrm{CS}, \mathrm{OBS}$ & Italy & 249; 229 (92\%); 34.50 (12.21) & NA & $\begin{array}{l}\text { March 18- } \\
21,2020\end{array}$ & $\begin{array}{l}\text { Anxiety and } \\
\text { fear }\end{array}$ & FCV-19S, HADS \\
\hline $\begin{array}{l}\text { Sutin et al. } \\
(2020)[147]^{1}\end{array}$ & $\mathrm{CS}, \mathrm{OBS}$ & USA & $\begin{array}{l}2094 ; 1024(48.9 \%)^{d} ; 51.03 \\
(16.58)\end{array}$ & $\begin{array}{l}\text { overweight }(n=706) \text {; } \\
\text { obesity }(n=587)\end{array}$ & $\begin{array}{l}\text { mid-March, } \\
2020\end{array}$ & $\begin{array}{l}\text { Anxiety and } \\
\text { fear }\end{array}$ & $\begin{array}{l}13 \text { items 5-P } \\
L^{a}\end{array}$ \\
\hline \multirow[t]{5}{*}{$\begin{array}{l}\text { Tan W et al. } \\
(2020)[80]^{1}\end{array}$} & \multirow[t]{5}{*}{$\mathrm{CS}, \mathrm{OBS}$} & \multirow[t]{5}{*}{ China } & \multirow[t]{5}{*}{$673 ; 172^{d}\left(25.6 \%^{d}\right) ; 30.8(7.4)$} & \multirow[t]{5}{*}{ NA } & \multirow[t]{5}{*}{$\begin{array}{l}\text { February } \\
24-252,020\end{array}$} & $\begin{array}{l}\text { Anxiety and } \\
\text { fear }\end{array}$ & $\begin{array}{l}\text { DASS-21 } \\
\text { Anxiety }\end{array}$ \\
\hline & & & & & & $\begin{array}{l}\text { Depressive } \\
\text { symptoms }\end{array}$ & $\begin{array}{l}\text { DASS-21 } \\
\text { Depression }\end{array}$ \\
\hline & & & & & & Stress & DASS-21 Stress \\
\hline & & & & & & $\begin{array}{l}\text { Sleep-related } \\
\text { symptoms }\end{array}$ & $|S|$ \\
\hline & & & & & & PTSS & IES-R \\
\hline \multirow[t]{4}{*}{$\begin{array}{l}\text { Tian et al. } \\
(2020)[81]^{1}\end{array}$} & \multirow[t]{4}{*}{$\mathrm{CS}, \mathrm{OBS}$} & \multirow[t]{4}{*}{ China } & \multirow[t]{4}{*}{ 1060; 511 (48.2\%); 35.01 (12.8) } & \multirow[t]{4}{*}{$\begin{array}{l}\mathrm{HCW}(n=42) \\
\text { students }(n=330)\end{array}$} & \multirow{4}{*}{$\begin{array}{l}\text { January } 31- \\
\text { February 02, } \\
2020\end{array}$} & $\begin{array}{l}\text { Anxiety and } \\
\text { fear }\end{array}$ & SCL-90 Anxiety \\
\hline & & & & & & $\begin{array}{l}\text { Depressive } \\
\text { symptoms }\end{array}$ & $\begin{array}{l}\text { SCL-90 } \\
\text { Depression }\end{array}$ \\
\hline & & & & & & $\begin{array}{l}\text { Psychological } \\
\text { distress }\end{array}$ & SCL-90 GSI \\
\hline & & & & & & $\begin{array}{l}\text { Other } \\
\text { outcomes }\end{array}$ & $\begin{array}{l}\text { SCL-90 } \\
\text { subscales }\end{array}$ \\
\hline \multirow{2}{*}{$\begin{array}{l}\text { Tsipropoulou } \\
\text { et al. (2020) } \\
{[82]^{1}}\end{array}$} & \multirow[t]{2}{*}{$\mathrm{CS}, \mathrm{OBS}$} & \multirow[t]{2}{*}{ Greece } & \multirow[t]{2}{*}{$\begin{array}{l}\text { 2970; } 2153(72.5 \%) ; ~ N A \\
\text { (mode: } 18-30 \text { years [52\%]) }\end{array}$} & \multirow[t]{2}{*}{ NA } & \multirow[t]{2}{*}{ NA } & $\begin{array}{l}\text { Anxiety and } \\
\text { fear }\end{array}$ & $\begin{array}{l}\text { FCV-19S, GAD- } \\
7\end{array}$ \\
\hline & & & & & & $\begin{array}{l}\text { Depressive } \\
\text { symptoms }\end{array}$ & PHQ-9 \\
\hline \multirow[t]{3}{*}{$\begin{array}{l}\text { Tull et al. } \\
(2020)[79]^{1}\end{array}$} & \multirow[t]{3}{*}{$\mathrm{CS}, \mathrm{OBS}$} & \multirow[t]{3}{*}{ USA } & \multirow[t]{3}{*}{$500 ; 235^{d}(47 \%) ; 40(11.6)$} & \multirow[t]{3}{*}{ NA } & \multirow{3}{*}{$\begin{array}{l}\text { March 27- } \\
\text { April 5, } \\
2020\end{array}$} & $\begin{array}{l}\text { Anxiety and } \\
\text { fear }\end{array}$ & $\begin{array}{l}\text { DASS-21 } \\
\text { Anxiety, SHAI }\end{array}$ \\
\hline & & & & & & $\begin{array}{l}\text { Depressive } \\
\text { symptoms }\end{array}$ & $\begin{array}{l}\text { DASS-21 } \\
\text { Depression }\end{array}$ \\
\hline & & & & & & Stress & DASS-21 Stress \\
\hline \multirow[t]{4}{*}{$\begin{array}{l}\text { Voitsidis et al. } \\
(2020)[83]^{1}\end{array}$} & $\mathrm{CS}, \mathrm{OBS}$ & Greece & $\begin{array}{l}\text { 2363; } 1800(76.2 \%) ; \text { NA } \\
\text { (mode: } 18-30 \text { years [55\%]) }\end{array}$ & NA & $\begin{array}{l}\text { April 10-13, } \\
2020\end{array}$ & $\begin{array}{l}\text { Anxiety and } \\
\text { fear }\end{array}$ & a \\
\hline & & & & & & $\begin{array}{l}\text { Depressive } \\
\text { symptoms }\end{array}$ & PHQ-2 \\
\hline & & & & & & $\begin{array}{l}\text { Sleep-related } \\
\text { symptoms }\end{array}$ & AIS \\
\hline & & & & & & $\begin{array}{l}\text { Other } \\
\text { outcomes }\end{array}$ & IUS-12, JGLS \\
\hline $\begin{array}{l}\text { Wang C et al. } \\
(2020 a)\end{array}$ & $\begin{array}{l}2 \mathrm{CS} \\
\text { (repeated), }\end{array}$ & China & $\begin{array}{l}1738 \text { not counting } \\
\text { participants in both surveys; }\end{array}$ & NA & $\begin{array}{l}\text { January } 31- \\
\text { February } 2\end{array}$ & $\begin{array}{l}\text { Anxiety and } \\
\text { fear }\end{array}$ & $\begin{array}{l}\text { DASS-21 } \\
\text { Anxiety }\end{array}$ \\
\hline $\begin{array}{l}\text { C et al. } \\
\text { (2020b) }[85]^{1}\end{array}$ & & & $\begin{array}{l}333 \text { in both } \\
\text { 1st survey: } 1210 ; 814^{d} \text { or } 878^{d} \\
\text { (67.3\%); NA (mode: } 21.4-30.8\end{array}$ & & $\begin{array}{l}2020 \text { and } \\
\text { February } \\
\text { 28-March 1, }\end{array}$ & $\begin{array}{l}\text { Depressive } \\
\text { symptoms }\end{array}$ & $\begin{array}{l}\text { DASS-21 } \\
\text { Depression }\end{array}$ \\
\hline & & & years [53.1\%]) & & 2020 & Stress & DASS-21 Stress \\
\hline & & & & & & PTSS & IES-R \\
\hline
\end{tabular}


Table 1 Study characteristics of included main studies (Continued)

\begin{tabular}{|c|c|c|c|c|c|c|c|}
\hline Study & $\begin{array}{l}\text { Study } \\
\text { design }\end{array}$ & Country & $\begin{array}{l}\text { Sample size; female: No. } \\
\text { (\%); age: mean (SD) or } \\
\text { alternative information on } \\
\text { age (eg, mode) }\end{array}$ & Subgroups & $\begin{array}{l}\text { Survey } \\
\text { period }\end{array}$ & $\begin{array}{l}\text { Assessed } \\
\text { Outcomes }\end{array}$ & $\begin{array}{l}\text { Instruments } \\
\text { or scales }\end{array}$ \\
\hline & & & $\begin{array}{l}\text { NA (mode: } 21.4-30.8 \text { years } \\
{[46.5 \%] \text { ) }}\end{array}$ & & & & \\
\hline $\begin{array}{l}\text { Wang } \mathrm{H} \text { et al. } \\
(2020)[86]^{1}\end{array}$ & $\mathrm{CS}, \mathrm{OBS}$ & China & 1599; 1068 (66.8\%); 33.9 (12.3) & NA & $\begin{array}{l}\text { February 1- } \\
4,2020\end{array}$ & $\begin{array}{l}\text { Psychological } \\
\text { distress }\end{array}$ & $\mathrm{K}-6$ \\
\hline \multirow{2}{*}{$\begin{array}{l}\text { Wang } Y \text { et al. } \\
(2020)[87]^{1,2}\end{array}$} & \multirow[t]{2}{*}{$\mathrm{CS}, \mathrm{OBS}$} & \multirow[t]{2}{*}{ China } & \multirow[t]{2}{*}{ 600; 333 (55.5\%); 34 (12) } & \multirow[t]{2}{*}{ NA } & \multirow{2}{*}{$\begin{array}{l}\text { February 6- } \\
9,2020\end{array}$} & Anxiety & SAS \\
\hline & & & & & & $\begin{array}{l}\text { Depressive } \\
\text { symptoms }\end{array}$ & SDS \\
\hline $\begin{array}{l}\text { Yang } \mathrm{H} \text { et al. } \\
(2020)[88]^{1}\end{array}$ & $\begin{array}{l}\text { CS } \\
\text { (repeated), } \\
\text { OBS }\end{array}$ & China & $\begin{array}{l}\text { during COVID-19: } 3000 ; 1500^{d} \\
(50 \%) ; 34.7 \text { (NA) }\end{array}$ & NA & $\begin{array}{l}\text { end of } \\
\text { December } \\
2019 \text { and } \\
\text { mid- } \\
\text { February, } \\
2020\end{array}$ & $\begin{array}{l}\text { Other } \\
\text { outcomes }\end{array}$ & $\begin{array}{l}\text { Emotional } \\
\text { well-being } \\
\text { (Kahneman } \\
\text { and Deaton, } \\
\text { 2010) }\end{array}$ \\
\hline \multirow[t]{3}{*}{$\begin{array}{l}\text { Yuan R et al. } \\
(2020)[89]^{1}\end{array}$} & \multirow[t]{3}{*}{$\mathrm{CS}, \mathrm{OBS}$} & \multirow[t]{3}{*}{ China } & \multirow{3}{*}{$\begin{array}{l}\text { parents of children } \\
\text { hospitalised during the } \\
\text { epidemic (EH): } 50 ; 31\left(62 \%{ }^{\mathrm{d}}\right) \text {; } \\
36.80(5.20) \text { parents of } \\
\text { children hospitalised during } \\
\text { the non-epidemic period } \\
\text { (NEH): } 50 ; 26\left(52 \%^{\mathrm{d}}\right) ; 37.22 \\
\text { (5.40) }\end{array}$} & \multirow[t]{3}{*}{$\begin{array}{l}\mathrm{EH}(n=50)^{\mathrm{b}}, \mathrm{NEH} \\
(n=50)^{\mathrm{b}}\end{array}$} & \multirow[t]{3}{*}{ NA } & Anxiety & $\begin{array}{l}\text { HADS Anxiety, } \\
\text { VDAS }\end{array}$ \\
\hline & & & & & & $\begin{array}{l}\text { Depressive } \\
\text { symptoms }\end{array}$ & $\begin{array}{l}\text { HADS } \\
\text { Depression }\end{array}$ \\
\hline & & & & & & $\begin{array}{l}\text { Other } \\
\text { Outcomes }\end{array}$ & SF-36 \\
\hline \multirow{2}{*}{$\begin{array}{l}\text { Zhang SX } \\
\text { et al. (2020a) } \\
{[90]^{1} \text {; Zhang }} \\
\text { SX et al. } \\
\text { (2020b) [91] }\end{array}$} & \multirow[t]{2}{*}{$\mathrm{CS}, \mathrm{OBS}$} & \multirow[t]{2}{*}{ China } & \multirow[t]{2}{*}{ 369; 165 (44.7\%); 36.6 (10.5) } & \multirow[t]{2}{*}{ NA } & \multirow[t]{2}{*}{$\begin{array}{l}\text { February } \\
20-21,2020\end{array}$} & $\begin{array}{l}\text { Psychological } \\
\text { Distress }\end{array}$ & K6 \\
\hline & & & & & & $\begin{array}{l}\text { Other } \\
\text { outcomes }\end{array}$ & SF12, SWLS \\
\hline $\begin{array}{l}\text { Zhang } Y \\
\text { et al. }(2020) \\
{[92]^{1,2}}\end{array}$ & $\mathrm{CS}, \mathrm{OBS}$ & China & 263; 157 (60\%); 37.7 (14.0) & NA & $\begin{array}{l}\text { January } 28- \\
\text { February 05, } \\
2020\end{array}$ & PTSS & IES \\
\hline \multirow{2}{*}{$\begin{array}{l}\text { Zhou SJ et al. } \\
(2020)[93]^{1}\end{array}$} & \multirow[t]{2}{*}{ CS, OBS } & \multirow[t]{2}{*}{ China } & \multirow{2}{*}{$\begin{array}{l}\text { 8079; } 4326 \text { (53.5\%); NA } \\
\text { (median: 16, minimum 12, } \\
\text { maximum 18years) }\end{array}$} & \multirow{2}{*}{$\begin{array}{l}100 \% \text { senior high } \\
\text { school students }^{\mathrm{b}}\end{array}$} & \multirow{2}{*}{$\begin{array}{l}\text { March 8-15, } \\
2020\end{array}$} & Anxiety & GAD-7 \\
\hline & & & & & & $\begin{array}{l}\text { Depressive } \\
\text { symptoms }\end{array}$ & PHQ-9 \\
\hline \multicolumn{8}{|c|}{ Healthcare workers } \\
\hline $\begin{array}{l}\text { Abdessater } \\
\text { et al. }(2020) \\
{[94]^{1}}\end{array}$ & $\mathrm{CS}, \mathrm{OBS}$ & France & $\begin{array}{l}275 ; 91^{d}(33 \%) \text { or } 83^{d}(30 \%) \\
\text { ambiguous data; } 29.5(0.47)\end{array}$ & $100 \%$ urologists & $\begin{array}{l}\text { March 27- } \\
30,2020\end{array}$ & Stress & a \\
\hline $\begin{array}{l}\text { Ahmed et al. } \\
(2020)[95]^{1}\end{array}$ & $\mathrm{CS}, \mathrm{OBS}$ & $\begin{array}{l}\text { multinational } \\
\text { (Pakistan > } \\
\text { Saudi Arabia } \\
>\text { others) }\end{array}$ & $\begin{array}{l}\text { 650; } 490 \text { (75\%); NA (mode: } \\
\text { 20-30 years [54\%]) }\end{array}$ & $100 \%$ dentists & $\begin{array}{l}\text { March 10- } \\
17,2020\end{array}$ & Anxiety & 8 binary items ${ }^{a}$ \\
\hline $\begin{array}{l}\text { Alhaj et al. } \\
(2020)[96]^{1}\end{array}$ & $\mathrm{CS}, \mathrm{OBS}$ & $\begin{array}{l}\text { multinational } \\
\text { (Canada, USA, } \\
\text { others) }\end{array}$ & $\begin{array}{l}52 ; 14(27 \%) ; \text { NA (mode: }<30 \\
\text { years }[69 \%])\end{array}$ & $100 \%$ surgeons & $\begin{array}{l}\text { April 14-28, } \\
2020\end{array}$ & $\begin{array}{l}\text { Psychological } \\
\text { distress }\end{array}$ & $\begin{array}{l}\text { Affection of } \\
\text { mental health } \\
\text { (binary single }_{\text {item) }^{\mathrm{a}}}\end{array}$ \\
\hline \multirow{4}{*}{$\begin{array}{l}\text { Amerio et al. } \\
(2020)[97]^{1}\end{array}$} & \multirow[t]{4}{*}{$\mathrm{CS}, \mathrm{OBS}$} & \multirow[t]{4}{*}{ Italy } & \multirow[t]{4}{*}{$131 ; 63$ (48.1\%); 52.3 (12.2) } & \multirow{4}{*}{$\begin{array}{l}\text { 100\% physicians } \\
\text { (general practitioners) }\end{array}$} & \multirow{4}{*}{$\begin{array}{l}\text { March 15- } \\
\text { April 15, } \\
2020\end{array}$} & Anxiety & GAD-7 \\
\hline & & & & & & $\begin{array}{l}\text { Depressive } \\
\text { symptoms }\end{array}$ & PHQ-9 \\
\hline & & & & & & $\begin{array}{l}\text { Sleep-related } \\
\text { symptoms }\end{array}$ & $|S|$ \\
\hline & & & & & & $\begin{array}{l}\text { Other } \\
\text { outcomes }\end{array}$ & SF-12 \\
\hline \multirow{3}{*}{$\begin{array}{l}\text { Badahdah } \\
\text { et al. }(2020) \\
{[98]^{1}}\end{array}$} & \multirow[t]{3}{*}{$\mathrm{CS}, \mathrm{OBS}$} & Oman & $194 ; 116^{\mathrm{d}}(60 \%) ; 40.72(8.53)$ & $100 \%$ physicians & early April & Anxiety & GAD-7 \\
\hline & & & & & & Stress & PSS-10 \\
\hline & & & & & & Other & $\mathrm{WHO}-5^{\mathrm{c}}$ \\
\hline
\end{tabular}


Table 1 Study characteristics of included main studies (Continued)

\begin{tabular}{|c|c|c|c|c|c|c|c|}
\hline Study & $\begin{array}{l}\text { Study } \\
\text { design }\end{array}$ & Country & $\begin{array}{l}\text { Sample size; female: No. } \\
\text { (\%); age: mean (SD) or } \\
\text { alternative information on } \\
\text { age (eg, mode) }\end{array}$ & Subgroups & $\begin{array}{l}\text { Survey } \\
\text { period }\end{array}$ & $\begin{array}{l}\text { Assessed } \\
\text { Outcomes }\end{array}$ & $\begin{array}{l}\text { Instruments } \\
\text { or scales }\end{array}$ \\
\hline & & & & & & outcomes & \\
\hline \multirow[t]{2}{*}{$\begin{array}{l}\text { Bohlken et al. } \\
\text { (2020) [99] }\end{array}$} & \multirow[t]{2}{*}{$\mathrm{CS}, \mathrm{OBS}$} & \multirow[t]{2}{*}{ Germany } & \multirow[t]{2}{*}{ 396; NA; 165 (42\%); 56.9 (7.6) } & \multirow[t]{2}{*}{$100 \%$ physicians } & \multirow[t]{2}{*}{$\begin{array}{l}\text { April 1-6, } \\
2020\end{array}$} & $\begin{array}{l}\text { Anxiety and } \\
\text { fear }\end{array}$ & $\begin{array}{l}\text { Single items 5- } \\
P L^{a}\end{array}$ \\
\hline & & & & & & $\begin{array}{l}\text { Sleep } \\
\text { disorders }\end{array}$ & $\begin{array}{l}\text { Single item 5-P } \\
\mathrm{LS}^{\mathrm{a}}\end{array}$ \\
\hline $\begin{array}{l}\text { Cai } \mathrm{H} \text { et al. } \\
(2020)[100]^{1,2}\end{array}$ & $\mathrm{CS}, \mathrm{OBS}$ & China & 534; 367 (69\%); 36.4 (16.18) & $\begin{array}{l}\text { physicians }(n=233) \text {, } \\
\text { nurses }(n=248)\end{array}$ & $\begin{array}{l}\text { January- } \\
\text { March, } 2020\end{array}$ & $\begin{array}{l}\text { Anxiety and } \\
\text { fear }\end{array}$ & $\begin{array}{l}\text { Single items 4- } \\
P L S^{a}\end{array}$ \\
\hline \multirow[t]{4}{*}{$\begin{array}{l}\text { Cai W et al. } \\
(2020)[101]^{1}\end{array}$} & \multirow[t]{4}{*}{$\mathrm{CS}, \mathrm{OBS}$} & \multirow[t]{4}{*}{ China } & \multirow{4}{*}{$\begin{array}{l}\text { whole sample: } \\
\text { 1521; } 1149\left(75.5 \%{ }^{\mathrm{d}}\right) \text {; NA } \\
\text { (mode: } 18-30 \text { years, [43.5\%]) }\end{array}$} & \multirow[t]{4}{*}{$\begin{array}{l}\text { physicians }(n=511) \text {, } \\
\text { nurses }(n=546)\end{array}$} & \multirow[t]{4}{*}{ NA } & $\begin{array}{l}\text { Anxiety and } \\
\text { fear }\end{array}$ & SCL-90 anxiety \\
\hline & & & & & & $\begin{array}{l}\text { Depressive } \\
\text { symptoms }\end{array}$ & $\begin{array}{l}\text { SCL-90 } \\
\text { depression }\end{array}$ \\
\hline & & & & & & $\begin{array}{l}\text { Psychological } \\
\text { distress }\end{array}$ & $\begin{array}{l}\text { SCL-90 positive } \\
\text { items }\end{array}$ \\
\hline & & & & & & $\begin{array}{l}\text { Other } \\
\text { outcomes }\end{array}$ & $\begin{array}{l}\text { SCL-90 } \\
\text { subscales, CD- } \\
\text { RISC, SSRS }\end{array}$ \\
\hline \multirow[t]{5}{*}{$\begin{array}{l}\text { Chew et al. } \\
(2020)[102]^{1}\end{array}$} & \multirow[t]{5}{*}{$\mathrm{CS}, \mathrm{OBS}$} & \multirow{5}{*}{$\begin{array}{l}\text { multinational } \\
\text { (Singapore, } \\
\text { India) }\end{array}$} & \multirow[t]{5}{*}{$\begin{array}{l}\text { 906; } 583 \text { (64.3\%); NA (median } \\
\text { [IQR]: } 29 \text { [25-35] years) }\end{array}$} & \multirow{5}{*}{$\begin{array}{l}\text { physicians }(n=268), \\
\text { nurses }(n=355) \text {, allied } \\
\text { healthcare } \\
\text { professionals }(n=96) \text {, } \\
\text { non-HCW }(n=187)\end{array}$} & \multirow{5}{*}{$\begin{array}{l}\text { February } \\
\text { 19-April 17, } \\
2020\end{array}$} & $\begin{array}{l}\text { Anxiety and } \\
\text { fear }\end{array}$ & $\begin{array}{l}\text { DASS-21 } \\
\text { anxiety }\end{array}$ \\
\hline & & & & & & $\begin{array}{l}\text { Depressive } \\
\text { symptoms }\end{array}$ & $\begin{array}{l}\text { DASS-21 } \\
\text { depression }\end{array}$ \\
\hline & & & & & & Stress & DASS-21 stress \\
\hline & & & & & & $\begin{array}{l}\text { Sleep-related } \\
\text { symptoms }\end{array}$ & $\begin{array}{l}\text { Single item 4-P } \\
L^{\mathrm{a}}\end{array}$ \\
\hline & & & & & & PTSS & IES-R \\
\hline $\begin{array}{l}\text { Consolo et al. } \\
(2020)[103]^{1}\end{array}$ & $\mathrm{CS}, \mathrm{OBS}$ & Italy & 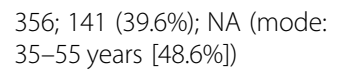 & $100 \%$ dentists & $\begin{array}{l}\text { April 2-21, } \\
2020\end{array}$ & $\begin{array}{l}\text { Anxiety and } \\
\text { fear }\end{array}$ & GAD-7 \\
\hline \multirow[t]{2}{*}{$\begin{array}{l}\text { Gan et al. } \\
(2020)[104]^{1}\end{array}$} & \multirow[t]{2}{*}{$\mathrm{CS}, \mathrm{OBS}$} & \multirow[t]{2}{*}{ China } & \multirow[t]{2}{*}{$\begin{array}{l}\text { 11,183; } 10,811(96.7 \%) ; \mathrm{NA} \\
\text { (mode: } 20-29 \text { years) }\end{array}$} & \multirow[t]{2}{*}{$100 \%$ nurses } & \multirow[t]{2}{*}{$\begin{array}{l}\text { February } 4- \\
10,2020\end{array}$} & $\begin{array}{l}\text { Anxiety and } \\
\text { fear }\end{array}$ & VAS on anxiety \\
\hline & & & & & & Stress & VAS on stress \\
\hline \multirow{2}{*}{$\begin{array}{l}\text { Huang JZ } \\
\text { et al. }(2020) \\
{[105]^{1,2}}\end{array}$} & \multirow[t]{2}{*}{$\mathrm{CS}, \mathrm{OBS}$} & \multirow[t]{2}{*}{ China } & \multirow[t]{2}{*}{$\begin{array}{l}\text { 230; } 187(81.3 \%) ; \text { NA (mode: } \\
\text { 30-39years [53\%]) }\end{array}$} & \multirow[t]{2}{*}{$\begin{array}{l}\text { physicians }(n=70) \text {, } \\
\text { nurses }(n=160)\end{array}$} & \multirow[t]{2}{*}{$\begin{array}{l}\text { February } 7- \\
14,2020\end{array}$} & $\begin{array}{l}\text { Anxiety and } \\
\text { fear }\end{array}$ & SAS \\
\hline & & & & & & PTSS & PTSD-SS \\
\hline \multirow[t]{4}{*}{$\begin{array}{l}\text { Kang et al. } \\
(2020)[106]^{1,2}\end{array}$} & CS, OBS & China & $\begin{array}{l}\text { 994; } 850(85.5 \%) \text {; NA (mode: } \\
30-40 \text { years [63.4\%]) }\end{array}$ & $\begin{array}{l}\text { physicians }(n=183) \text {, } \\
\text { nurses }(n=811)\end{array}$ & $\begin{array}{l}\text { January } 29- \\
\text { February } 4\end{array}$ & $\begin{array}{l}\text { Anxiety and } \\
\text { fear }\end{array}$ & GAD-7 ${ }^{e}$ \\
\hline & & & & & & $\begin{array}{l}\text { Depressive } \\
\text { symptoms }\end{array}$ & PHQ-9 ${ }^{e}$ \\
\hline & & & & & & $\begin{array}{l}\text { Sleep-related } \\
\text { symptoms }\end{array}$ & $|S|^{e}$ \\
\hline & & & & & & PTSS & IES-R \\
\hline $\begin{array}{l}\text { Khusid et al. } \\
(2020)[107]^{1}\end{array}$ & $\mathrm{CS}, \mathrm{OBS}$ & USA & 332; 117 (35\%); $30.5(2.6)$ & $100 \%$ urologists & $\begin{array}{l}\text { April 7-11, } \\
2020\end{array}$ & $\begin{array}{l}\text { Anxiety and } \\
\text { fear }\end{array}$ & 2 items 5-P $L S^{a}$ \\
\hline & & & & & & $\begin{array}{l}\text { Depressive } \\
\text { symptoms }\end{array}$ & 2 items 5-P $L S^{a}$ \\
\hline $\begin{array}{l}\text { Lai et al. } \\
(2020)[18]^{1,2}\end{array}$ & $\mathrm{CS}, \mathrm{OBS}$ & China & $\begin{array}{l}\text { 1257; } 964(76.7 \%) ; ~ N A ~(m o d e: ~ \\
26-40 \text { years [64.7\%]) }\end{array}$ & $\begin{array}{l}\text { physicians }(n=493) \text {, } \\
\text { nurses }(n=764)\end{array}$ & $\begin{array}{l}\text { January 29- } \\
\text { February 3, }\end{array}$ & $\begin{array}{l}\text { Anxiety and } \\
\text { fear }\end{array}$ & GAD-7 \\
\hline & & & & & & $\begin{array}{l}\text { Depressive } \\
\text { symptoms }\end{array}$ & PHQ-9 \\
\hline & & & & & & $\begin{array}{l}\text { Sleep-related } \\
\text { symptoms }\end{array}$ & $|S|$ \\
\hline & & & & & & PTSS & IES \\
\hline
\end{tabular}


Table 1 Study characteristics of included main studies (Continued)

\begin{tabular}{|c|c|c|c|c|c|c|c|}
\hline Study & $\begin{array}{l}\text { Study } \\
\text { design }\end{array}$ & Country & $\begin{array}{l}\text { Sample size; female: No. } \\
\text { (\%); age: mean (SD) or } \\
\text { alternative information on } \\
\text { age (eg, mode) }\end{array}$ & Subgroups & $\begin{array}{l}\text { Survey } \\
\text { period }\end{array}$ & $\begin{array}{l}\text { Assessed } \\
\text { Outcomes }\end{array}$ & $\begin{array}{l}\text { Instruments } \\
\text { or scales }\end{array}$ \\
\hline \multirow[t]{2}{*}{$\begin{array}{l}\text { Mo et al. } \\
(2020)[108]^{1,2}\end{array}$} & \multirow[t]{2}{*}{$\mathrm{CS}, \mathrm{OBS}$} & \multirow[t]{2}{*}{ China } & \multirow[t]{2}{*}{ 180; 162 (90\%); 32.71 (6.52) } & \multirow[t]{2}{*}{ NA } & \multirow{2}{*}{$\begin{array}{l}\text { end of } \\
\text { February } \\
2020\end{array}$} & $\begin{array}{l}\text { Anxiety and } \\
\text { fear }\end{array}$ & SAS \\
\hline & & & & & & Stress & sos \\
\hline \multirow[t]{2}{*}{$\begin{array}{l}\text { Pu et al. } \\
(2020)[109]^{1}\end{array}$} & \multirow[t]{2}{*}{$\mathrm{CS}, \mathrm{OBS}$} & \multirow[t]{2}{*}{ China } & \multirow[t]{2}{*}{ 867: $829\left(95.6 \%{ }^{d}\right) ; 30.8(7.1)$} & \multirow[t]{2}{*}{$100 \%$ nurses } & \multirow[t]{2}{*}{ NA } & $\begin{array}{l}\text { Anxiety and } \\
\text { fear }\end{array}$ & SAS \\
\hline & & & & & & $\begin{array}{l}\text { Other } \\
\text { outcomes }\end{array}$ & TAF \\
\hline \multirow[t]{5}{*}{$\begin{array}{l}\text { Rossi et al. } \\
(2020)[110]^{1}\end{array}$} & \multirow[t]{5}{*}{$\mathrm{CS}, \mathrm{OBS}$} & \multirow[t]{5}{*}{ Italy } & \multirow[t]{5}{*}{ 1379; 1064 (77.2\%); 39.0 (6.0) } & \multirow{5}{*}{$\begin{array}{l}\text { physicians }(n=433) \text {, } \\
\text { general practitioners } \\
(n=86) \text {, nurses }(n= \\
472)\end{array}$} & \multirow[t]{5}{*}{$\begin{array}{l}\text { March 27- } \\
31,2020\end{array}$} & $\begin{array}{l}\text { Anxiety and } \\
\text { fear }\end{array}$ & GAD-7 \\
\hline & & & & & & $\begin{array}{l}\text { Depressive } \\
\text { symptoms }\end{array}$ & PHQ-9 \\
\hline & & & & & & Stress & PSS \\
\hline & & & & & & $\begin{array}{l}\text { Sleep-related } \\
\text { symptoms }\end{array}$ & $|S|$ \\
\hline & & & & & & PTSS & GPS-PTSD \\
\hline $\begin{array}{l}\text { Sahu et al. } \\
(2020)[111]^{1}\end{array}$ & $\mathrm{CS}, \mathrm{OBS}$ & India & $\begin{array}{l}611 ; \mathrm{NA} ; \mathrm{NA} \text { (mode: } 30-40 \\
\text { years, } n=192[31 \cdot 4 \%])\end{array}$ & $\begin{array}{l}100 \% \text { orthopedic } \\
\text { surgeons }\end{array}$ & $\begin{array}{l}\text { March 31- } \\
\text { April 4, } \\
2020\end{array}$ & Stress & Single-item ${ }^{a}$ \\
\hline $\begin{array}{l}\text { Shacham } \\
\text { et al. (2020) } \\
{[112]^{1}}\end{array}$ & $\mathrm{CS}, \mathrm{OBS}$ & Israel & 338; 198 (586\%); 46.39 (11.2) & $\begin{array}{l}\text { dentists }(n=198), \\
\text { dental hygienists }(n= \\
\left.140^{d}\right)\end{array}$ & $\begin{array}{l}\text { March 30- } \\
\text { April 10, } \\
2020\end{array}$ & $\begin{array}{l}\text { Psychological } \\
\text { distress }\end{array}$ & K-6 \\
\hline $\begin{array}{l}\text { Suleiman } \\
\text { et al. }(2020) \\
{[113]^{1}}\end{array}$ & $\mathrm{CS}, \mathrm{OBS}$ & Jordan & 308; 113 (36.7\%); 30.3 (5.8) & 100\% physicians & $\begin{array}{l}\text { March 23- } \\
27,2020\end{array}$ & $\begin{array}{l}\text { Anxiety and } \\
\text { fear }\end{array}$ & $\begin{array}{l}\text { Binary single } \\
\text { items }^{\mathrm{a}}\end{array}$ \\
\hline \multirow[t]{4}{*}{$\begin{array}{l}\text { Tan B et al. } \\
(2020)[114]^{1}\end{array}$} & \multirow[t]{4}{*}{$\mathrm{CS}, \mathrm{OBS}$} & \multirow[t]{4}{*}{ Singapore } & \multirow[t]{4}{*}{$\begin{array}{l}\text { 470; } 321 \text { (68.3\%); NA (median: } \\
\text { 31, IQR: 28-36 years) }\end{array}$} & \multirow{4}{*}{$\begin{array}{l}\text { physicians }(n=135) \text {, } \\
\text { nurses }(n=161) \text {, allied } \\
\text { hospital personnel } \\
(n=174)\end{array}$} & \multirow{4}{*}{$\begin{array}{l}\text { February } \\
\text { 19-March } \\
13,2020\end{array}$} & $\begin{array}{l}\text { Anxiety and } \\
\text { fear }\end{array}$ & $\begin{array}{l}\text { DASS-21 } \\
\text { anxiety }\end{array}$ \\
\hline & & & & & & $\begin{array}{l}\text { Depressive } \\
\text { symptoms }\end{array}$ & $\begin{array}{l}\text { DASS-21 } \\
\text { depression }\end{array}$ \\
\hline & & & & & & Stress & DASS-21 stress \\
\hline & & & & & & PTSS & IES-R \\
\hline \multirow[t]{3}{*}{$\begin{array}{l}\text { Wang S et al. } \\
(2020)[115]^{1}\end{array}$} & \multirow[t]{3}{*}{$\mathrm{CS}, \mathrm{OBS}$} & \multirow[t]{3}{*}{ China } & \multirow[t]{3}{*}{ 123; 111 (90\%); 33.75 (8.41) } & \multirow{3}{*}{$\begin{array}{l}100 \% \text { pediatricians; } \\
\text { physicians }(n=48) \\
\text { nurses }(n=75)\end{array}$} & \multirow{3}{*}{$\begin{array}{l}\text { January } 30- \\
\text { February 07, } \\
2020\end{array}$} & $\begin{array}{l}\text { Anxiety and } \\
\text { fear }\end{array}$ & SAS \\
\hline & & & & & & $\begin{array}{l}\text { Depressive } \\
\text { symptoms }\end{array}$ & SDS \\
\hline & & & & & & $\begin{array}{l}\text { Sleep-related } \\
\text { symptoms }\end{array}$ & PSQI \\
\hline \multirow[t]{6}{*}{$\begin{array}{l}\text { Wu K et al. } \\
(2020)[116]^{1}\end{array}$} & $\begin{array}{l}\text { CS, OBS, } \\
\text { controlled }\end{array}$ & China & $\begin{array}{l}\text { experimental group: } 60 ; 44 \\
(73 \%) ; 33.5(12.4)\end{array}$ & $\begin{array}{l}\text { COVID-19 hospital } \\
(n=60) \text {, non- }\end{array}$ & NA & $\begin{array}{l}\text { Anxiety and } \\
\text { fear }\end{array}$ & $\begin{array}{l}\text { SAS, SCL-90 } \\
\text { anxiety }\end{array}$ \\
\hline & & & $\begin{array}{l}\text { comparison group: 60; } 45 \\
(75 \%) 33.8 \text { (11.9) }\end{array}$ & $\begin{array}{l}\text { designated hospital = } \\
\text { comparison group } \\
(n=60)\end{array}$ & & $\begin{array}{l}\text { Depressive } \\
\text { symptoms }\end{array}$ & $\begin{array}{l}\text { SCL-90 } \\
\text { depression, } \\
\text { SDS }\end{array}$ \\
\hline & & & & & & $\begin{array}{l}\text { Sleep-related } \\
\text { symptoms }\end{array}$ & PSQI \\
\hline & & & & & & PTSS & PCL-C \\
\hline & & & & & & $\begin{array}{l}\text { Psychological } \\
\text { distress }\end{array}$ & $\begin{array}{l}\text { SCL-90 total } \\
\text { score }\end{array}$ \\
\hline & & & & & & $\begin{array}{l}\text { Other } \\
\text { outcomes }\end{array}$ & $\begin{array}{l}\text { SCL-90 } \\
\text { subscales }\end{array}$ \\
\hline $\begin{array}{l}\text { Xiao et al. } \\
(2020 \mathrm{a})\end{array}$ & $\mathrm{CS}, \mathrm{OBS}$ & China & 180; 129 (71.7\%); 32.31 (4.88) & $\begin{array}{l}\text { physicians }(n=82) \\
\text { nurses }(n=98)\end{array}$ & $\begin{array}{l}\text { January- } \\
\text { February, }\end{array}$ & $\begin{array}{l}\text { Anxiety and } \\
\text { fear }\end{array}$ & SAS \\
\hline & & & & & & Sleep-related & PSQI \\
\hline
\end{tabular}


Table 1 Study characteristics of included main studies (Continued)

\begin{tabular}{|c|c|c|c|c|c|c|c|}
\hline Study & $\begin{array}{l}\text { Study } \\
\text { design }\end{array}$ & Country & $\begin{array}{l}\text { Sample size; female: No. } \\
\text { (\%); age: mean (SD) or } \\
\text { alternative information on } \\
\text { age (eg, mode) }\end{array}$ & Subgroups & $\begin{array}{l}\text { Survey } \\
\text { period }\end{array}$ & $\begin{array}{l}\text { Assessed } \\
\text { Outcomes }\end{array}$ & $\begin{array}{l}\text { Instruments } \\
\text { or scales }\end{array}$ \\
\hline & & & & & & symptoms & \\
\hline & & & & & & $\begin{array}{l}\text { Other } \\
\text { outcomes }\end{array}$ & $\begin{array}{l}\text { GSES, SASR, } \\
\text { SSRS }\end{array}$ \\
\hline \multirow[t]{3}{*}{$\begin{array}{l}\text { Xu J et al. } \\
\text { (2020) [118] }]^{1}\end{array}$} & \multirow[t]{3}{*}{$\begin{array}{l}\text { CS, OBS, } \\
\text { controlled }\end{array}$} & \multirow[t]{3}{*}{ China } & \multirow{3}{*}{$\begin{array}{l}\text { outbreak period: } 60 ; 38 \\
(63.3 \%) ; 36.68 \text { (9.67) } \\
\text { 'post-epidemic': 60; } 32 \\
(53.3 \%) ; 35.77 \text { (7.06) }\end{array}$} & \multirow[t]{3}{*}{$100 \%$ surgeons } & \multirow{3}{*}{$\begin{array}{l}\text { January 28- } \\
\text { February 29, } \\
2020 \text { and } \\
\text { March 2-21, } \\
2020\end{array}$} & $\begin{array}{l}\text { Anxiety and } \\
\text { fear }\end{array}$ & $\begin{array}{l}\text { 'Anxiety scale', } \\
\text { dream anxiety } \\
\text { score }\end{array}$ \\
\hline & & & & & & $\begin{array}{l}\text { Depressive } \\
\text { symptoms }\end{array}$ & $\begin{array}{l}\text { 'Depression } \\
\text { score' }\end{array}$ \\
\hline & & & & & & $\begin{array}{l}\text { Other } \\
\text { outcomes }\end{array}$ & SF-36 \\
\hline \multirow[t]{2}{*}{$\begin{array}{l}\text { Yin et al. } \\
\text { (2020) [119] }\end{array}$} & \multirow[t]{2}{*}{$\mathrm{CS}, \mathrm{OBS}$} & \multirow[t]{2}{*}{ China } & \multirow{2}{*}{$\begin{array}{l}371 ; 228(61.5 \%) ; 35.3(9.5) \\
\text { physicians: NA } \\
\text { nurses: NA }\end{array}$} & \multirow[t]{2}{*}{$\begin{array}{l}\text { physicians }(n=67) \\
\text { nurses }(n=264)\end{array}$} & \multirow[t]{2}{*}{$\begin{array}{l}\text { February } \\
01-05,2020\end{array}$} & $\begin{array}{l}\text { Sleep-related } \\
\text { symptoms }\end{array}$ & PSQI \\
\hline & & & & & & PTSS & PCL-5 \\
\hline \multirow{4}{*}{$\begin{array}{l}\text { Zhang C } \\
\text { et al. }(2020) \\
{[120]^{1}}\end{array}$} & \multirow[t]{4}{*}{$\mathrm{CS}, \mathrm{OBS}$} & \multirow[t]{4}{*}{ China } & \multirow{4}{*}{$\begin{array}{l}\text { 1563; } 1293\left(83 \%{ }^{\mathrm{d}}\right) \text {; NA (mode: } \\
\text { 26-40 years, } n=495\left[31.7 \%{ }^{\mathrm{d}}\right] \text { ) } \\
\text { physicians: NA } \\
\text { nurses: NA }\end{array}$} & \multirow{4}{*}{$\begin{array}{l}\text { physicians }(n=454) \text {, } \\
\text { nurses }(n=984), \\
\text { administrative staff } \\
(n=30) \text {, other } \\
\text { medical staff }(n=95)\end{array}$} & \multirow{4}{*}{$\begin{array}{l}\text { January 29- } \\
\text { February 03, } \\
2020\end{array}$} & $\begin{array}{l}\text { Anxiety and } \\
\text { fear }\end{array}$ & GAD-7 \\
\hline & & & & & & $\begin{array}{l}\text { Depressive } \\
\text { symptoms }\end{array}$ & PHQ-9 \\
\hline & & & & & & $\begin{array}{l}\text { Sleep-related } \\
\text { symptoms }\end{array}$ & $|S|$ \\
\hline & & & & & & PTSS & IES-R \\
\hline \multirow{4}{*}{$\begin{array}{l}\text { Zhang SX } \\
\text { et al. (2020c) } \\
{[121]^{1}}\end{array}$} & \multirow[t]{4}{*}{$\mathrm{CS}, \mathrm{OBS}$} & \multirow[t]{4}{*}{ Iran } & \multirow[t]{4}{*}{$304 ; 178$ (58.6\%); 35.1 (9.1) } & \multirow[t]{4}{*}{ NA } & \multirow[t]{4}{*}{$\begin{array}{l}\text { April 5-20, } \\
2020\end{array}$} & $\begin{array}{l}\text { Anxiety and } \\
\text { fear }\end{array}$ & GAD-2 ${ }^{d}$ \\
\hline & & & & & & $\begin{array}{l}\text { Depressive } \\
\text { symptoms }\end{array}$ & PHQ-2 ${ }^{d}$ \\
\hline & & & & & & $\begin{array}{l}\text { Psychological } \\
\text { distress }\end{array}$ & K6 \\
\hline & & & & & & $\begin{array}{l}\text { Other } \\
\text { outcomes }\end{array}$ & SF-12 \\
\hline \multirow[t]{2}{*}{$\begin{array}{l}\text { Zhu J et al. } \\
\text { (2020) [122] }\end{array}$} & \multirow[t]{2}{*}{ CS, OBS } & \multirow[t]{2}{*}{ China } & \multirow[t]{2}{*}{$\begin{array}{l}\text { 156; } 137(83 \%) ; 34.16(8.06) \\
\text { physicians: } 79 ; 51^{d}\left(65 \%^{d}\right)\end{array}$} & \multirow[t]{2}{*}{$\begin{array}{l}\text { physicians }(n=79) \text {, } \\
\text { nurses }(n=86)\end{array}$} & \multirow[t]{2}{*}{$\begin{array}{l}\text { February 1- } \\
29,2020\end{array}$} & $\begin{array}{l}\text { Anxiety and } \\
\text { fear }\end{array}$ & SAS \\
\hline & & & & & & $\begin{array}{l}\text { Depressive } \\
\text { symptoms }\end{array}$ & SDS \\
\hline \multicolumn{8}{|l|}{ Patients } \\
\hline $\begin{array}{l}\text { Cai } X \text { et al. } \\
\text { (2020) [123] }\end{array}$ & CS, OBS & China & $126 ; 66(52.4 \%) ; 45.7(14.0)$ & $\begin{array}{l}\text { 100\% cured COVID-19 } \\
\text { patients }\end{array}$ & $\begin{array}{l}\text { March 2-12, } \\
2020\end{array}$ & $\begin{array}{l}\text { Anxiety and } \\
\text { fear }\end{array}$ & SAS \\
\hline (2020) [124] ${ }^{1}$ & & & & & & $\begin{array}{l}\text { Depressive } \\
\text { symptoms }\end{array}$ & SDS \\
\hline & & & & & & PTSS & PTSD-SS \\
\hline $\begin{array}{l}\text { Durankus } \\
\text { et al. (2020) }\end{array}$ & $\mathrm{CS}, \mathrm{OBS}$ & Turkey & $260 ; 260$ (100\%); $29.6(3.8)$ & $\begin{array}{l}100 \% \text { pregnant } \\
\text { women }\end{array}$ & NA & $\begin{array}{l}\text { Anxiety and } \\
\text { fear }\end{array}$ & $\mathrm{BAl}$ \\
\hline & & & & & & $\begin{array}{l}\text { Depressive } \\
\text { symptoms }\end{array}$ & EPDS, BDI \\
\hline & & & & & & $\begin{array}{l}\text { Psychological } \\
\text { distress }\end{array}$ & $\begin{array}{l}\text { Single item 11- } \\
\mathrm{P}\left\llcorner\mathrm{S}^{\mathrm{a}}\right.\end{array}$ \\
\hline $\begin{array}{l}\text { Li X et al. } \\
(2020)[126]^{1}\end{array}$ & $\mathrm{CS}, \mathrm{OBS}$ & China & $76 ; 35$ (46\%); $36(15)$ & $\begin{array}{l}\text { suspected COVID-19 } \\
\text { patients }\end{array}$ & $\begin{array}{l}\text { January } 31- \\
\text { February 22, }\end{array}$ & $\begin{array}{l}\text { Anxiety and } \\
\text { fear }\end{array}$ & HAMA \\
\hline & & & & & & $\begin{array}{l}\text { Depressive } \\
\text { symptoms }\end{array}$ & HAMD \\
\hline $\begin{array}{l}\text { Liu X et al. } \\
(2020 a)[42]^{1}\end{array}$ & $\mathrm{CS}, \mathrm{OBS}$ & China & $\begin{array}{l}\text { COVID-19 suspected patients: } \\
21 ; 12(57.1 \%) ; 43.1(2.6): \\
\text { not COVID-19 suspected }\end{array}$ & $\begin{array}{l}\text { 100\% schizophrenia } \\
\text { patients; COVID-19 } \\
\text { suspected patients }\end{array}$ & $\begin{array}{l}\text { January } 30- \\
\text { February } 21 \\
2020\end{array}$ & $\begin{array}{l}\text { Anxiety and } \\
\text { fear }\end{array}$ & HAMA \\
\hline
\end{tabular}


Table 1 Study characteristics of included main studies (Continued)

\begin{tabular}{|c|c|c|c|c|c|c|c|}
\hline Study & $\begin{array}{l}\text { Study } \\
\text { design }\end{array}$ & Country & $\begin{array}{l}\text { Sample size; female: No. } \\
\text { (\%); age: mean (SD) or } \\
\text { alternative information on } \\
\text { age (eg, mode) }\end{array}$ & Subgroups & $\begin{array}{l}\text { Survey } \\
\text { period }\end{array}$ & $\begin{array}{l}\text { Assessed } \\
\text { Outcomes }\end{array}$ & $\begin{array}{l}\text { Instruments } \\
\text { or scales }\end{array}$ \\
\hline \multirow{6}{*}{$\begin{array}{l}\text { Wu Y et al. } \\
(2020)[127]^{1,3}\end{array}$} & & & $\begin{array}{l}\text { patients: 30; } 15 \text { (50\%); } 45.0 \\
(9.2)\end{array}$ & $\begin{array}{l}\text { ( } n=21), \text { not COVID- } \\
19 \text { suspected patients }\end{array}$ & & $\begin{array}{l}\text { Depressive } \\
\text { symptoms }\end{array}$ & HAMD \\
\hline & & & & & & Stress & PSS \\
\hline & & & & & & $\begin{array}{l}\text { Sleep-related } \\
\text { symptoms }\end{array}$ & PSQI \\
\hline & & & & & & $\begin{array}{l}\text { Other } \\
\text { outcomes }\end{array}$ & PANSS \\
\hline & \multirow[t]{2}{*}{$\begin{array}{l}\text { CS, OBS, } \\
\text { controlled }\end{array}$} & \multirow[t]{2}{*}{ China } & \multirow{2}{*}{$\begin{array}{l}4124 ; 4124\left(100 \%{ }^{d}\right), \text { NA } \\
\text { (median: } 30, \text { range }=17-32 \\
\text { years) }\end{array}$} & \multirow{2}{*}{$\begin{array}{l}\text { 100\% pregnant } \\
\text { women; } \\
\text { before (group 1: } n= \\
\text { 2839)/after (group 2: } \\
n=1284 \text { ) January 20, } \\
2020\end{array}$} & \multirow{2}{*}{$\begin{array}{l}\text { January 1- } \\
\text { February 9, } \\
2020\end{array}$} & $\begin{array}{l}\text { Anxiety and } \\
\text { fear }\end{array}$ & EPDS-3A \\
\hline & & & & & & $\begin{array}{l}\text { Depressive } \\
\text { symptoms }\end{array}$ & EPDS \\
\hline \multirow[t]{2}{*}{$\begin{array}{l}\text { Xu H et al. } \\
(2020)[128]^{1}\end{array}$} & \multirow[t]{2}{*}{$\mathrm{CS}, \mathrm{OBS}$} & \multirow[t]{2}{*}{ China } & \multirow[t]{2}{*}{$\begin{array}{l}\text { 350; } 199(54.1 \%) ; \text { NA (mode: } \\
40-60 \text { years [51\%]) }\end{array}$} & \multirow[t]{2}{*}{$\begin{array}{l}100 \% \text { lung cancer } \\
\text { patients }\end{array}$} & \multirow[t]{2}{*}{$\begin{array}{l}\text { March 4-6, } \\
2020\end{array}$} & $\begin{array}{l}\text { Depressive } \\
\text { symptoms }\end{array}$ & Single item ${ }^{a}$ \\
\hline & & & & & & $\begin{array}{l}\text { Sleep-related } \\
\text { symptoms }\end{array}$ & Single item ${ }^{a}$ \\
\hline $\begin{array}{l}\text { Yassa et al. } \\
(2020)[129]^{1}\end{array}$ & $\mathrm{CS}, \mathrm{OBS}$ & Turkey & $172 ; 172(100 \%) ; 27.5(5.3)$ & $\begin{array}{l}100 \% \text { pregnant } \\
\text { women }\end{array}$ & $\begin{array}{l}\text { ten days } \\
\text { after first } \\
\text { confirmed } \\
\text { COVID-19 } \\
\text { death in } \\
\text { Turkey }\end{array}$ & $\begin{array}{l}\text { Anxiety and } \\
\text { fear }\end{array}$ & $\begin{array}{l}\text { Single ternary } \\
\text { item }^{\mathrm{a}}\end{array}$ \\
\hline \multicolumn{8}{|l|}{ Mixed groups } \\
\hline \multirow[t]{2}{*}{$\begin{array}{l}\text { Büntzel et al. } \\
\text { (2020) [130] }\end{array}$} & \multirow[t]{2}{*}{$\mathrm{CS}, \mathrm{OBS}$} & \multirow[t]{2}{*}{ Germany } & \multirow[t]{2}{*}{$\begin{array}{l}\text { 193; NA; NA (mode: > } 60 \\
\text { years) }\end{array}$} & \multirow{2}{*}{$\begin{array}{l}\text { physicians }(n=47), \\
\text { cancer patients }(n= \\
\text { 146) }\end{array}$} & \multirow[t]{2}{*}{$\begin{array}{l}\text { April 16-19, } \\
2020\end{array}$} & $\begin{array}{l}\text { Anxiety and } \\
\text { fear }\end{array}$ & Single item ${ }^{a}$ \\
\hline & & & & & & Stress & Single item ${ }^{a}$ \\
\hline \multirow[t]{4}{*}{$\begin{array}{l}\text { Guo et al. } \\
(2020)[131]^{1}\end{array}$} & \multirow[t]{4}{*}{$\begin{array}{l}\text { CS, OBS, } \\
\text { controlled }\end{array}$} & \multirow[t]{4}{*}{ China } & \multirow{4}{*}{$\begin{array}{l}\text { P:103; } 44(42.7 \%) ; 42.5(12.5) \\
\text { control (GP): 103; } 49 \text { (47.6\%); } \\
41.5 \text { (13.1) }\end{array}$} & \multirow{4}{*}{$\begin{array}{l}\text { COVID-19 patients } \\
(n=103), \text { not infected } \\
\text { control group }(n= \\
\text { 103) }\end{array}$} & \multirow[t]{4}{*}{$\begin{array}{l}\text { February } \\
10-28,2020\end{array}$} & $\begin{array}{l}\text { Anxiety and } \\
\text { fear }\end{array}$ & GAD-7 \\
\hline & & & & & & $\begin{array}{l}\text { Depressive } \\
\text { symptoms }\end{array}$ & PHQ-9 \\
\hline & & & & & & Stress & PSS-10 \\
\hline & & & & & & PTSS & PCL-5 \\
\hline \multirow[t]{5}{*}{$\begin{array}{l}\text { Hao F et al. } \\
(2020)[132]^{1}\end{array}$} & \multirow[t]{5}{*}{$\begin{array}{l}\text { CS, OBS, } \\
\text { controlled }\end{array}$} & \multirow[t]{5}{*}{ China } & \multirow{5}{*}{$\begin{array}{l}\text { P: } 76 ; 51 \text { (37.1\%); } 32.8 \text { (11.8); } \\
\text { control (GP): 109; } 68 \text { (62.4\%); } \\
33.1 \text { (11.2) }\end{array}$} & \multirow{5}{*}{$\begin{array}{l}\text { psychiatric patients } \\
(n=76), \text { control } \\
\text { group }(n=109)\end{array}$} & \multirow[t]{5}{*}{$\begin{array}{l}\text { February } \\
19-22,2020\end{array}$} & $\begin{array}{l}\text { Anxiety and } \\
\text { fear }\end{array}$ & $\begin{array}{l}\text { DASS-21 } \\
\text { anxiety }\end{array}$ \\
\hline & & & & & & $\begin{array}{l}\text { Depressive } \\
\text { symptoms }\end{array}$ & $\begin{array}{l}\text { DASS-21 } \\
\text { depression }\end{array}$ \\
\hline & & & & & & Stress & DASS-21 stress \\
\hline & & & & & & $\begin{array}{l}\text { Sleep-related } \\
\text { symptoms }\end{array}$ & $|S|$ \\
\hline & & & & & & PTSS & IES-R \\
\hline $\begin{array}{l}\text { Hao X et al. } \\
(2020)[133]^{1}\end{array}$ & $\begin{array}{l}\text { CS, OBS, } \\
\text { controlled }\end{array}$ & China & $\begin{array}{l}\text { P: } 252 ; 132^{d}\left(52.4 \%^{d}\right) ; 29.3 \\
(11.6) ; \\
\text { control (GP): } 252 ; 132^{d} \\
\left(52.4 \%^{d}\right) ; 29.4(11.5)\end{array}$ & $\begin{array}{l}\text { epilepsy patients }(n= \\
252) \text {, control group } \\
(n=252)\end{array}$ & $\begin{array}{l}\text { February 1- } \\
29,2020\end{array}$ & $\begin{array}{l}\text { Psychological } \\
\text { distress }\end{array}$ & $\mathrm{K}-6$ \\
\hline \multirow{3}{*}{$\begin{array}{l}\text { Huang Y } \\
\text { et al. }(2020) \\
{[134]^{1,2}}\end{array}$} & $\mathrm{CS}, \mathrm{OBS}$ & China & 7236; 3952 (54.6\%); 35.3 (5.6) & $\begin{array}{l}\mathrm{GP}(n=4986), \mathrm{HCW} \\
(n=2250)\end{array}$ & $\begin{array}{l}\text { February 3- } \\
17,2020\end{array}$ & $\begin{array}{l}\text { Anxiety and } \\
\text { fear }\end{array}$ & GAD-7 \\
\hline & & & & & & $\begin{array}{l}\text { Depressive } \\
\text { symptoms }\end{array}$ & CES-D \\
\hline & & & & & & $\begin{array}{l}\text { Sleep-related } \\
\text { symptoms }\end{array}$ & PSQI \\
\hline lasevoli et al. & $\mathrm{CS}, \mathrm{OBS}$ & Italy & $461 ; N A ; N A$ & psychiatric patients & April 13-17, & Anxiety and & GAD-7 \\
\hline
\end{tabular}


Table 1 Study characteristics of included main studies (Continued)

\begin{tabular}{|c|c|c|c|c|c|c|c|}
\hline Study & $\begin{array}{l}\text { Study } \\
\text { design }\end{array}$ & Country & $\begin{array}{l}\text { Sample size; female: No. } \\
\text { (\%); age: mean (SD) or } \\
\text { alternative information on } \\
\text { age (eg, mode) }\end{array}$ & Subgroups & $\begin{array}{l}\text { Survey } \\
\text { period }\end{array}$ & $\begin{array}{l}\text { Assessed } \\
\text { Outcomes }\end{array}$ & $\begin{array}{l}\text { Instruments } \\
\text { or scales }\end{array}$ \\
\hline \multirow[t]{4}{*}{$(2020)[135]^{1}$} & \multirow[t]{4}{*}{ controlled } & & \multirow{4}{*}{$\begin{array}{l}\text { P: 205; NA; NA } \\
\text { caregivers: 51; NA; NA } \\
\text { control (GP): 205; NA; NA }\end{array}$} & \multirow{4}{*}{$\begin{array}{l}(n=205), \text { caregivers } \\
(n=51) \text {, non- } \\
\text { psychiatric persons } \\
(n=205)\end{array}$} & \multirow[t]{4}{*}{2020} & fear & \\
\hline & & & & & & $\begin{array}{l}\text { Depressive } \\
\text { symptoms }\end{array}$ & PHQ-9 \\
\hline & & & & & & Stress & PSS \\
\hline & & & & & & $\begin{array}{l}\text { Other } \\
\text { outcomes }\end{array}$ & SPEQ \\
\hline $\begin{array}{l}\text { Jin YH et al. } \\
(2020)[136]^{1}\end{array}$ & CS, OBS & China & $\begin{array}{l}\text { 103; } 64 \text { (62.1\%); NA (median } \\
\text { [IQR]: } 35 \text { [14.0]) }\end{array}$ & $\begin{array}{l}100 \% \text { infected with } \\
\text { SARS-CoV-2; physi- } \\
\text { cians, nurses }\end{array}$ & $\begin{array}{l}\text { February } \\
15-29,2020\end{array}$ & $\begin{array}{l}\text { Anxiety and } \\
\text { fear }\end{array}$ & $\begin{array}{l}\text { Single item } \\
\text { multiple } \\
\text { choice }^{a}\end{array}$ \\
\hline $\begin{array}{l}\text { Ko et al. } \\
(2020)[137]^{1}\end{array}$ & $\mathrm{CS}, \mathrm{OBS}$ & Taiwan & 1904; 1282 (67.3\%); 38.0 (10.8) & $\begin{array}{l}\mathrm{GP}(n=N A), \mathrm{HCW} \\
(n=N A)\end{array}$ & $\begin{array}{l}\text { April 10-20, } \\
2020\end{array}$ & $\begin{array}{l}\text { Other } \\
\text { outcomes }\end{array}$ & $\begin{array}{l}\text { Psychological } \\
\text { wellbeing } \\
\text { (single item 5- }^{\text {P LS) }}{ }^{\mathrm{a}}\end{array}$ \\
\hline $\begin{array}{l}\text { Li Z et al. } \\
(2020)[138]^{1,2}\end{array}$ & $\mathrm{CS}, \mathrm{OBS}$ & China & $\begin{array}{l}740 ; 128(59.8 \%) ; 25 \text { (IQR: } 22- \\
38.3 \text { years] }\end{array}$ & $\begin{array}{l}\mathrm{GP}(n=214), \mathrm{HCW} \\
(n=526)\end{array}$ & $\begin{array}{l}\text { February } \\
17-21,2020\end{array}$ & PTSS & $\begin{array}{l}\text { Vicarious } \\
\text { Traumatization } \\
\text { Questionnaire }\end{array}$ \\
\hline \multirow[t]{2}{*}{$\begin{array}{l}\text { Lu W et al. } \\
(2020)[139]^{1,2}\end{array}$} & \multirow[t]{2}{*}{ CS, OBS } & \multirow[t]{2}{*}{ China } & \multirow[t]{2}{*}{$\begin{array}{l}2299 ; 1785 \text { (77.6\%); NA (78\% } \\
<40 \text { years) }\end{array}$} & \multirow[t]{2}{*}{$\begin{array}{l}\mathrm{HCW}(n=2042), \mathrm{GP} \\
(n=257)\end{array}$} & \multirow[t]{2}{*}{$\begin{array}{l}\text { February } \\
25.26,2020\end{array}$} & $\begin{array}{l}\text { Anxiety and } \\
\text { fear }\end{array}$ & $\begin{array}{l}\text { HAMA, NRS on } \\
\text { fear }\end{array}$ \\
\hline & & & & & & $\begin{array}{l}\text { Depressive } \\
\text { symptoms }\end{array}$ & HAMD \\
\hline \multirow[t]{2}{*}{$\begin{array}{l}\text { Ni et al. } \\
(2020)[140]^{1}\end{array}$} & \multirow[t]{2}{*}{ CS, OBS } & \multirow[t]{2}{*}{ China } & \multirow{2}{*}{$\begin{array}{l}\text { total: 1791; NA; NA } \\
\text { GP: } 1577 ; 1218^{\text {d }}(60.8 \%) ; \text { NA } \\
\text { (mode: } 18-34 \text { years [38.6\%]) } \\
\text { HCW: } 214 ; 147^{d} \text { (68.8\%); NA } \\
\text { (mode: } 18-34 \text { years [58.9\%]) }\end{array}$} & \multirow[t]{2}{*}{$\begin{array}{l}\mathrm{GP}(n=1577), \mathrm{HCW} \\
(n=214)\end{array}$} & \multirow[t]{2}{*}{$\begin{array}{l}\text { February } \\
18.24,2020\end{array}$} & $\begin{array}{l}\text { Anxiety and } \\
\text { fear }\end{array}$ & GAD-2 \\
\hline & & & & & & $\begin{array}{l}\text { Depressive } \\
\text { symptoms }\end{array}$ & PHQ-2 \\
\hline \multirow[t]{2}{*}{$\begin{array}{l}\text { Sanchez et al. } \\
(2020)[67]^{1}\end{array}$} & \multirow[t]{2}{*}{$\mathrm{CS}, \mathrm{OBS}$} & \multirow[t]{2}{*}{ USA } & \multirow[t]{2}{*}{$1051 ; 0$ (0\%); 35 (15.83) } & \multirow{2}{*}{$\begin{array}{l}100 \% \text { men who have } \\
\text { sex with men; HIV- } \\
\text { patients ( } n=122)\end{array}$} & \multirow[t]{2}{*}{$\begin{array}{l}\text { April 2-13, } \\
2020\end{array}$} & $\begin{array}{l}\text { Anxiety and } \\
\text { fear }\end{array}$ & Single item ${ }^{a}$ \\
\hline & & & & & & $\begin{array}{l}\text { Other } \\
\text { outcomes }\end{array}$ & $\begin{array}{l}\text { Quality of life } \\
\text { (single item) }^{\text {a }}\end{array}$ \\
\hline \multirow[t]{2}{*}{$\begin{array}{l}\text { Wu W et al. } \\
(2020)[141]^{1}\end{array}$} & \multirow[t]{2}{*}{$\mathrm{CS}, \mathrm{OBS}$} & \multirow[t]{2}{*}{ China } & \multirow{2}{*}{$\begin{array}{l}\text { 4268; } 2930^{\mathrm{d}}\left(68.7 \%^{\mathrm{d}}\right) ; \mathrm{NA} \\
\text { HCW: } 2110 ; 1598^{\mathrm{d}}\left(76 \%^{\mathrm{d}}\right) ; \mathrm{NA} \\
\text { Students: } 2158 ; 1332(62 \%) ; \\
\text { NA }\end{array}$} & \multirow[t]{2}{*}{$\begin{array}{l}\text { students }(n=2158) \\
\text { HCW }(n=2110)\end{array}$} & \multirow[t]{2}{*}{$\begin{array}{l}\text { February } \\
10-21,2020\end{array}$} & $\begin{array}{l}\text { Anxiety and } \\
\text { fear }\end{array}$ & Single item ${ }^{a}$ \\
\hline & & & & & & $\begin{array}{l}\text { Sleep-related } \\
\text { symptoms }\end{array}$ & Single item ${ }^{a}$ \\
\hline \multirow[t]{2}{*}{$\begin{array}{l}\text { Yuan S et al. } \\
(2020)[142]^{1,2}\end{array}$} & \multirow[t]{2}{*}{ L, OBS } & \multirow[t]{2}{*}{ China } & $\begin{array}{l}\text { 939; } 582(61.98 \%) ; ~ N A ~(m o d e: ~ \\
\text { 18-39years [71.5\%]) }\end{array}$ & $\begin{array}{l}\text { HCW }(n=249) \\
\text { students }(n=312)\end{array}$ & $\begin{array}{l}2 \text { survey } \\
\text { periods in }\end{array}$ & $\begin{array}{l}\text { Sleep-related } \\
\text { symptoms }\end{array}$ & PSQI \\
\hline & & & & & $\begin{array}{l}\text { February, } \\
2020\end{array}$ & $\begin{array}{l}\text { Other } \\
\text { outcomes }\end{array}$ & SRQ \\
\hline $\begin{array}{l}\text { Zhang J et al. } \\
(2020)[143]^{1}\end{array}$ & CS, OBS & China & $\begin{array}{l}\text { 205; } 115\left(56.1 \%{ }^{\mathrm{d}}\right) \text {; NA (for } \\
\text { infected: } 46.9 \text { [15.4]; for }\end{array}$ & $\begin{array}{l}P \text {, infected }(n=57), \\
G P \text {, quarantined }(n=\end{array}$ & $\begin{array}{l}\text { February } \\
15-29,2020\end{array}$ & $\begin{array}{l}\text { Anxiety and } \\
\text { fear }\end{array}$ & GAD-7 \\
\hline & & & $\begin{array}{l}\text { quarantined: } 36.2 \text { [10.9]; for } \\
\text { general public: } 29.6 \text { [12.7]) }\end{array}$ & $\begin{array}{l}\text { G0), } \\
(n=98)\end{array}$ & & $\begin{array}{l}\text { Depressive } \\
\text { symptoms }\end{array}$ & PHQ-9 \\
\hline $\begin{array}{l}\text { Zhang WR } \\
\text { et al. (2020) }\end{array}$ & $\mathrm{CS}, \mathrm{OBS}$ & China & $\begin{array}{l}\text { 2182; } 1401(64.2 \%) \text { ) NA } \\
\text { (mode: 18-60 years [96.3\%]) }\end{array}$ & $\begin{array}{l}\mathrm{HCW}(n=927), \mathrm{GP} \\
(n=1255)\end{array}$ & $\begin{array}{l}\text { February } \\
\text { 19-March 6, }\end{array}$ & $\begin{array}{l}\text { Anxiety and } \\
\text { fear }\end{array}$ & GAD-2 \\
\hline & & & & & & $\begin{array}{l}\text { Depressive } \\
\text { symptoms }\end{array}$ & PHQ-2 \\
\hline & & & & & & $\begin{array}{l}\text { Sleep-related } \\
\text { symptoms }\end{array}$ & $|S|$ \\
\hline & & & & & & $\begin{array}{l}\text { Other } \\
\text { outcomes }\end{array}$ & $\begin{array}{l}\text { SCL-90-R } \\
\text { subscales }\end{array}$ \\
\hline $\begin{array}{l}\text { Zhu S et al. } \\
(2020)[145]^{1}\end{array}$ & CS, OBS & China & $2279^{d} ; 1361^{d} ; N A$ & $\begin{array}{l}\text { HCW }(n=858), \mathrm{GP} \\
(n=1421)\end{array}$ & $\begin{array}{l}\text { Feb 12-Mar } \\
17,2020\end{array}$ & $\begin{array}{l}\text { Anxiety and } \\
\text { fear }\end{array}$ & GAD-7 \\
\hline & & & & & & Depressive & PHQ-9 \\
\hline
\end{tabular}


Table 1 Study characteristics of included main studies (Continued)

\begin{tabular}{|c|c|c|c|c|c|c|c|}
\hline Study & $\begin{array}{l}\text { Study } \\
\text { design }\end{array}$ & Country & $\begin{array}{l}\text { Sample size; female: No. } \\
(\%) ; \text { age: mean (SD) or } \\
\text { alternative information on } \\
\text { age (eg, mode) }\end{array}$ & Subgroups & $\begin{array}{l}\text { Survey } \\
\text { period }\end{array}$ & $\begin{array}{l}\text { Assessed } \\
\text { Outcomes }\end{array}$ & $\begin{array}{l}\text { Instruments } \\
\text { or scales }\end{array}$ \\
\hline & & & & & & symptoms & \\
\hline & & & & & & $\begin{array}{l}\text { Psychological } \\
\text { distress }\end{array}$ & SRQ-20 \\
\hline
\end{tabular}

\begin{abstract}
Abbreviations: AIS Athens Insomnia Scale, BAI Beck Anxiety Inventory, BDI Beck Depression Inventory, BDI(-II) Beck Depression Inventory(-II), BIP-Q5 Brief IIIness Perception Questionnaire 5, BRCS Brief Resilience Coping Scale, CD-RISC Connor-Davidson Resilience Scale, CES-D Center for Epidemiologic Studies Depression Scale, CoVGAD-7 Generalized Anxiety Disorder Scale-7 for COVID-19 Anxiety, CPDI CoViD-19 Peritraumatic Distress Index, CS cross-sectional, DASS-21 Depression Anxiety Stress Scale-21, DT Distress Thermometer, EPDS Edinburgh Postnatal Depression Scale, EPDS-3A Edinburgh Postnatal Depression Scale-Anxiety subscale, FACIT-Sp12 Functional Assessment of Chronic Illness Therapy-Spiritual Well-Being Scale, FCV-19S Fear of COVID-19 scale, GAD-2(-7) Generalized Anxiety Disorder Scale-2(/-7), GP general population, GPS-PTSD Global Psychotrauma Scale-posttraumatic stress disorder subscale, GSES General Self-Efficacy Scale, GSI Global Severity Index, HADS Hospital Anxiety and Depression Scale, HAI Health Anxiety Inventory, HAMA Hamilton Anxiety Rating Scale, HAMD Hamilton Depression Rating Scale, HCW healthcare workers, IES Impact of Event Scale, IES-R Impact of Event Scale-Revised, IQR interquartile range, ISI Insomnia Severity Index, IUS-12 Intolerance of Uncertainty Scale-Short Form, JGLS De Jong Gierveld Loneliness Scale, K-6(// 10) Kessler Psychological Distress Scale-6(/- 10), L longitudinal, MINI Mini International Neuropsychiatric Interview, MSPSS Multidimensional Scale of Perceived Social Support, NA not available, NRS Numeric Rating Scale, OBS observational, $P$ patients, PANSS Positive and Negative Syndrome Scale, $P C L-5(-C)$ Post-traumatic Stress Disorder Checklist-5(/-Civilian Version), PHQ-2//-4/-9/-15) Patient Health Questionnaire-2(/-4/-9/- 15), PROMIS-SFs Patient Reported Outcomes Measurement Information System short forms, PSQI Pittsburgh Sleep Quality Index, PSS(- 10) Perceived Stress Scale(- 10), PTSD-SS Post-traumatic Stress Disorder Self-rating Scale, PTSS post-traumatic stress symptoms, Ryff's PWB Ryff's Psychological Wellbeing Scales, SAS Self-Rating Anxiety Scale, SASR Stanford Acute Stress Reaction, SCL-90 Symptom Checklist-90, SCS Self-Compassion Scale, SD standard deviation, SDQ Strengths and Difficulties Questionnaire, SDS Self-Rating Depression Scale, SF-12(/-36) Short Form 12 Health Survey, SHAI Short Health Anxiety Inventory, SOS Stress Overload Scale, SPEQ Specific Psychotic Experience Questionnaire, SRQ Stress Response Questionnaire, SRQ-20 20-item Self-Report Questionnaire, SSRS Social Support Rating Scale, SSS Somatic Symptom Scale, STAI-Y State Trait Anxiety Inventory-Y, SWLS Satisfaction With Life Scale, TAF Triage Assessment Form, TEMPS-A Temperament Evaluation of Memphis, Pisa, Paris and San Diego-Anxious, VAS Visual Analogue Scale, VDAS Van Dream Anxiety Scale, WHO-5 World Health Organization- Five Well-Being Index, WHOQOL-BREF abbreviated World Health Organization Quality of Life, 4-/5-/7-/11-P LS 4-/5-/6-/11-point Likert-scale

${ }^{a}$ developed by study authors

${ }^{\mathrm{b}}$ included in main analyses for general population but considered separately in subgroup-analyses

c in Gao J et al. WHO-5 is used to assess depressive symptoms, in Badahdah et al. it is used to assess psychological distress

${ }^{d}$ not directly reported

e $\mathrm{k}$-means-clustering method for the 4 tools summarized to 'mental health'
\end{abstract}

The risk and protective factors narratively identified for each population are presented in Table 5 and eTables 14 and 15, with most of them being investigated in the general population, and few studies investigating protective factors at all. Most frequently named risk factors across the populations were pre-existing mental disorders, female sex, and concerns about COVID-19 infection, whereas most frequently reported protective factors were older age, good economic situation, and higher education.

\section{Discussion}

To our knowledge, this is the first systematic review and meta-analysis to assess the mental health impact of the SARS-CoV-2 pandemic in the general population, healthcare workers, and patients, by contrasting data from the early phase of the current pandemic with prepandemic data. We identified 104 independent studies, mainly in the general population, that suggest an increased prevalence of mental burden due to the SARS-CoV-2 pandemic. This finding is in line with previous reviews and meta-analyses that merely pooled the prevalence of or calculated the risk for mental burden in either one or several of these groups $[20,24,29,32,33,36]$.

On the other hand, the pairwise meta-analyses for 43 studies across the four primary outcomes revealed different results. Compared with prepandemic data, we only found an elevated level of some mental symptoms (anxiety, depression) due to the SARS-CoV-2 pandemic in the general population, but not of stress or sleeping problems.

Although healthcare workers were found to be a group at risk for mental health problems during the SARSCoV-2 pandemic $[18]^{1,2}[20,29,31,32]$, we identified no evidence for an increased mental burden during the early phase when comparing them with healthcare staff prior to the pandemic. Because of a (chronic) workrelated risk exposure in daily life [194], as a kind of 'stress inoculation', healthcare professionals might have learned effective strategies (eg, self-efficacy) helping them to cope more professionally with crises than other groups. In contrast to previous findings [20, 195], the level of COVID-19 patient contact did not affect the mental health impact.

Overall, the results of this review paint a more nuanced picture of the mental health consequences of the SARS-CoV-2 pandemic than previous reviews - an observation in line with stress resilience research that identified different trajectories of psychological adaptation after potentially traumatic events, ranging from no mental burden to severe mental illness [196, 197]. Indeed, a recent analysis of 523 healthy subjects from the German LORA study showed a decrease of perceived stress and stressor load while mental health improved during the eight-week measurement after lockdown, indicating that the pandemic and pandemic response may also have 
Table 2 Narrative synthesis of prevalence based on scores above cut-off values for different mental health outcomes

\begin{tabular}{|c|c|c|c|}
\hline & Number of studies ${ }^{a}$ & $\begin{array}{l}\text { Lowest reported } \\
\text { prevalence }(\%)\end{array}$ & $\begin{array}{l}\text { Highest reported } \\
\text { prevalence (\%) }\end{array}$ \\
\hline \multicolumn{4}{|l|}{ General population } \\
\hline $\begin{array}{l}\text { Anxiety, worries, } \\
\text { fear }\end{array}$ & $\begin{array}{l}24\left(18 \mathrm{GP},[45,47,49,50,52,57,63,66,68,69,71,77,93]^{1}[73,\right. \\
84,87]^{1,2} \\
\left.6 \mathrm{M}[132,140,145]^{1}[134,139,144]^{1,2}\right)\end{array}$ & $0.67(63)$ & $64.0(46)$ \\
\hline $\begin{array}{l}\text { Depressive } \\
\text { symptoms }\end{array}$ & $\begin{array}{l}18\left(13 \mathrm{GP}[45,49,50,52,57,63,66,68,69,71,93]^{1}[84,87]^{1,2}\right. \\
\left.5 \mathrm{M}[132,140,145]^{1}[139,144]^{1,2}\right)\end{array}$ & $0.9(89)$ & $48.3(48)$ \\
\hline PTSS & $7\left(6 \mathrm{GP}[52,66,71]^{1}[59,84,92]^{1,2}, 1 \mathrm{M}[132]^{1}\right)$ & $7.0(51)$ & $53.8(55)$ \\
\hline $\begin{array}{l}\text { Sleep-related } \\
\text { symptoms }\end{array}$ & $6\left(3\right.$ GP $\left.[71,83]^{1}[84]^{1,2}, 3 \mathrm{M}[132]^{1}[134,144]^{1,2}\right)$ & $0.9(89)$ & $37.6(131)$ \\
\hline Stress & $5\left(4 \mathrm{GP}[66,68,71,83]^{1}, 1 \mathrm{M}[132]^{1}\right)$ & $0.9(89)$ & $67.9(55)$ \\
\hline $\begin{array}{l}\text { Psychological } \\
\text { distress }\end{array}$ & 7 (5 GP $\left.[41,45,58,65,81]^{1}, 2 \mathrm{M}[133,136]^{1}\right)$ & $1.6(90)$ & $65.2(112)$ \\
\hline \multicolumn{4}{|l|}{ Healthcare workers } \\
\hline $\begin{array}{l}\text { Anxiety, worries, } \\
\text { fear }\end{array}$ & $\begin{array}{l}22\left(14 \mathrm{HCW}[99,95,113,102,103,110,115,120,122,121]^{1}[18,\right. \\
100,105,106]^{1,2} \\
\left.6 \mathrm{M}[130,140,145]^{1}[134,139,144]^{1,2}\right)\end{array}$ & $7.0(108)$ & $92.0(144)$ \\
\hline $\begin{array}{l}\text { Depressive } \\
\text { symptoms }\end{array}$ & $\begin{array}{l}14 \text { (9 HCW }[97,102,110,115,120,121,122]^{1}[18,106]^{1,2}, \\
\left.5 \mathrm{M}[140,145]^{1}[134,139,144]^{1,2}\right)\end{array}$ & $0.6(110)$ & $50.4(18)$ \\
\hline PTSS & 7 (HCW) $[102,110,119,120]^{1}[18,105,106]^{1,2}$ & $3.8(82)$ & $73.0(83)$ \\
\hline $\begin{array}{l}\text { Sleep-related } \\
\text { symptoms }\end{array}$ & $9\left(7 \mathrm{HCW}[99,102,110,115,120]^{1}[18,106]^{1,2}, 2 \mathrm{M}[134]^{1}[144]^{1,2}\right)$ & $8.27(127)$ & $38.0(108)$ \\
\hline Stress & $6\left(5 \mathrm{HCW}[94,102,110,111]^{1}[108]^{1,2}, 1 \mathrm{M}[130]^{1}\right)$ & $5.2(102)$ & $56.5(114)$ \\
\hline $\begin{array}{l}\text { Psychological } \\
\text { distress }\end{array}$ & 5 (4 HCW $\left.[96,101,112,121]^{1}, 1 \mathrm{M}[145]^{1}\right)$ & $11.1(101)$ & $90.4(145)$ \\
\hline \multicolumn{4}{|l|}{ Patients } \\
\hline $\begin{array}{l}\text { Anxiety, worries, } \\
\text { fear }\end{array}$ & $6\left(5 P[123,126,129,131,143]^{1}, 1 \mathrm{M}[132]^{1}\right)$ & $19.5(99)$ & $80.2(143)$ \\
\hline $\begin{array}{l}\text { Depressive } \\
\text { symptoms }\end{array}$ & $8\left(7 \mathrm{P}[123,125,126,128,131,143]^{1}[127]^{1,3}, 1 \mathrm{M}[132]^{1}\right)$ & $27.8(99)$ & $55.3(88)$ \\
\hline PTSS & $2\left(1 \mathrm{P}[123]^{1}, 1 \mathrm{M}[132]^{1}\right)$ & $31.0(84)$ & $43.4(89)$ \\
\hline $\begin{array}{l}\text { Sleep-related } \\
\text { symptoms }\end{array}$ & $2\left(1 \mathrm{P}[128]^{1}, 1 \mathrm{M} \mathrm{[132]^{1 } )}\right.$ & $27.6(89)$ & $66.3(97)$ \\
\hline Stress & $1\left(\mathrm{M}[132]^{1}\right)$ & $17.0(89)$ & \\
\hline $\begin{array}{l}\text { Psychological } \\
\text { distress }\end{array}$ & $1\left(\mathrm{M}[133]^{1}\right)$ & $13.1(90)$ & \\
\hline
\end{tabular}

Abbreviations: GP general population, $H C W$ healthcare workers, $M$ mixed samples, $P$ patients, $P$ TSS posttraumatic stress symptoms

${ }^{a}$ reporting prevalence rates for the respective mental health outcome

positive effects [198]. The number of studies reporting on protective factors in this review was rather limited, especially in healthcare workers and patients. However, these factors might also partly explain the heterogeneity of findings regarding mental health consequences. This is in line with positive aspects (eg, improved social relationships with close social contacts such as families) that were likewise reported for previous infectious disease outbreaks. The importance of taking a 'resilience perspective' in SARS-CoV-2 mental health research and investigating resilience factors has been pointed out previously [19, 22, 197, 199].
Several aspects must be considered when interpreting the results. First, the absence of evidence of effects in healthcare workers and patients in this review does not necessarily mean that there is evidence for the absence of effects of the SARS-CoV-2 pandemic on mental health in these groups. Second, for healthcare workers, the mental burden on individuals probably depends on the location of survey (eg, country, region) and how heavily the respective healthcare systems were burdened in the pandemic timeline (eg, number of hospitalized COVID-19 patients). Among the 13 included studies in meta-analyses for healthcare staff, we could only include 
Table 3 Results of main and sensitivity analyses in three populations

\begin{tabular}{|c|c|c|c|c|c|c|}
\hline Outcome & $\begin{array}{l}\text { Studies } \\
\text { (samples) }\end{array}$ & $\begin{array}{l}\mathrm{N} \\
\text { (pandemic) }\end{array}$ & $\begin{array}{l}\mathrm{N} \\
\text { (comp.) }\end{array}$ & $\begin{array}{l}\text { Standardized mean difference }(95 \% \\
\mathrm{CI})\end{array}$ & $1^{2}$ & $\begin{array}{l}95 \% \text { predic }^{2} \\
\text { interval }^{\mathrm{a}}\end{array}$ \\
\hline \multicolumn{7}{|l|}{ Main analyses } \\
\hline \multicolumn{7}{|c|}{ General population } \\
\hline Anxiety & $23(26)$ & 49,746 & 132,145 & $0.40(0.15-0.65)$ & $99 \%$ & $-0.87-1.67$ \\
\hline Depression & $25(28)$ & 60,213 & 183,747 & $0.67(0.07-1.27)$ & $100 \%$ & $-2.02-3.36$ \\
\hline Stress & $11(13)$ & 11,600 & 67,386 & $0.10(-0.30-0.50)$ & $100 \%$ & $-1.39-1.60$ \\
\hline $\begin{array}{l}\text { Sleep-related } \\
\text { symptoms }\end{array}$ & $4(4)$ & 3332 & 7635 & $0.74(-1.47-2.96)$ & $100 \%$ & $-3.68-5.17$ \\
\hline \multicolumn{7}{|c|}{ Healthcare workers } \\
\hline Anxiety & $13(14)$ & 5508 & 22,204 & $-0.08(-0.66-0.49)$ & $99 \%$ & $-1.75-1.58$ \\
\hline Depression & $7(8)$ & 2226 & 4605 & $-0.16(-0.59-0.26)$ & $97 \%$ & $-1.41-1.09$ \\
\hline Stress & $3(3)$ & 1570 & 2454 & $0.49(-0.60-1.57)$ & $99 \%$ & / \\
\hline $\begin{array}{l}\text { Sleep-related } \\
\text { symptoms }\end{array}$ & $4(5)$ & 554 & 20,024 & $0.83(-0.14-1.81)$ & $99 \%$ & $-1.54-3.21$ \\
\hline \multicolumn{7}{|l|}{ Patients } \\
\hline Anxiety & $6(6)$ & 1845 & 12,458 & $0.31(-0.07,0.69)$ & $93 \%$ & $-1.08-1.69$ \\
\hline Depression & $7(7)$ & 2138 & 24,444 & $0.48(-0.08-1.04)$ & $98 \%$ & $-1.58-2.53$ \\
\hline Stress & $4(4)$ & 435 & 10,061 & $-0.10(-0.81-0.61)$ & $98 \%$ & $-3.54-3.34$ \\
\hline $\begin{array}{l}\text { Sleep-related } \\
\text { symptoms }\end{array}$ & $2(2)$ & 127 & 298 & $-0.61(-1.75-0.54)$ & $96 \%$ & / \\
\hline
\end{tabular}

Sensitivity analysis - Quality of included pandemic studies (ie, exclusion of poor-quality studies)

\section{General population}

$\begin{array}{lllllll}\text { Anxiety } & 16(17) & 38,323 & 81,350 & 0.53(0.19-0.86) & 100 \% & -0.90-1.95 \\ \text { Depression } & 18(19) & 48,790 & 136,884 & 0.83(0.09-1.57) & 100 \%-2.17-3.82 \\ \begin{array}{l}\text { Stress } \\ \begin{array}{l}\text { Sleep-related } \\ \text { symptoms }\end{array}\end{array} & 7(8) & 9110 & 43,747 & 0.33(-0.19-0.84) & 100 \%-1.20-1.85 \\ \begin{array}{l}\text { Healthcare workers } \\ \text { Anxiety }\end{array} & 3(3) & 2659 & 6622 & 0.80(-1.34-2.94) & 100 \% / \\ \begin{array}{l}\text { Depression } \\ \text { Stress }\end{array} & 4(4) & 1655 & 4124 & -0.18(-0.78-0.41) & 97 \% & -1.30-0.94 \\ \text { Sleep-related } & 4(4) & 1655 & 2356 & 0.03(-0.42-0.47) & 90 \% & -0.73-0.79 \\ \text { symptoms } & 2(2) & 1376 & 1872 & -0.05(-0.37-0.26) & 95 \% / & /\end{array}$

symptoms

\section{Patients}

$\begin{array}{lllllll}\text { Anxiety } & 3(3) & 1461 & 11,116 & 0.45(-0.10-1.01) & 92 \% & / \\ \text { Depression } & 3(3) & 1461 & 21,934 & 0.21(-1.08-1.49) & 99 \% & / \\ \text { Stress } & 1(1) & 51 & 51 & 0.18(-0.21-0.57) & / & / \\ \text { Sleep-related } & 1(1) & 51 & 207 & -0.03(-0.33-0.28) & /\end{array}$

symptoms

Sensitivity analysis - Level of comparability between included pandemic studies and comparative studies (ie, exclusion of level-3 and level-4 studies)

\section{General population}

$\begin{array}{lllllll}\text { Anxiety } & 12(13) & 38,461 & 32,698 & 0.40(0.06-0.74) & 99 \% & -0.77-1.57 \\ \text { Depression } & 14(15) & 38,259 & 78,619 & 0.77(-0.23-1.77) & 100 \% & -2.72-4.25 \\ \text { Stress } & 7(8) & 8624 & 12,739 & -0.15(-0.76-0.46) & 99 \% & -1.84-1.53 \\ \begin{array}{l}\text { Sleep-related } \\ \text { symptoms }\end{array} & 2(2) & 2550 & 5609 & 1.54(-1.18-4.27) & 100 \% /\end{array}$


Table 3 Results of main and sensitivity analyses in three populations (Continued)

\begin{tabular}{|c|c|c|c|c|c|c|}
\hline Outcome & $\begin{array}{l}\text { Studies } \\
\text { (samples) }\end{array}$ & $\begin{array}{l}\mathrm{N} \\
\text { (pandemic) }\end{array}$ & $\begin{array}{l}\mathrm{N} \\
\text { (comp.) }\end{array}$ & $\begin{array}{l}\text { Standardized mean difference }(95 \% \\
\mathrm{Cl})\end{array}$ & $1^{2}$ & $\begin{array}{l}\text { 95\% prediction } \\
\text { interval }^{\mathrm{a}}\end{array}$ \\
\hline \multicolumn{7}{|c|}{ Healthcare workers } \\
\hline Anxiety & 7 (8) & 3147 & 9511 & $-0.54(-1.23-0.15)$ & $99 \%$ & $-2.11-1.03$ \\
\hline Depression & $4(5)$ & 546 & 2576 & $-0.38(-1.56-0.79)$ & $98 \%$ & $-2.60-1.84$ \\
\hline Stress & / & / & / & / & / & / \\
\hline $\begin{array}{l}\text { Sleep-related } \\
\text { symptoms }\end{array}$ & $3(4)$ & 423 & 19,804 & $1.01(-0.17-2.18)$ & $99 \%$ & $-1.61-3.63$ \\
\hline \multicolumn{7}{|l|}{ Patients } \\
\hline Anxiety & $4(4)$ & 1616 & 3184 & $0.23(-0.33-0.79)$ & $92 \%$ & $-2.47-2.93$ \\
\hline Depression & $4(4)$ & 1704 & 3205 & $0(-0.56-0.56)$ & $93 \%$ & $-2.69-2.70$ \\
\hline Stress & $2(2)$ & 127 & 217 & $0.15(-0.08-0.37)$ & $0 \%$ & / \\
\hline $\begin{array}{l}\text { Sleep-related } \\
\text { symptoms }\end{array}$ & $2(2)$ & 127 & 298 & $-0.61(-1.75-0.54)$ & $96 \%$ & / \\
\hline
\end{tabular}

Abbreviations: $\mathrm{Cl}$ confidence interval, comp. comparative studies, $I^{2}$ heterogeneity, $N$ sample size, pandemic included pandemic studies

a $95 \%$ prediction interval only calculated for meta-analyses with at least $k=4$ studies

a few studies from heavily burdened countries (eg, Italy: $\mathrm{k}=2$; Spain: $\mathrm{k}=0$; USA: $\mathrm{k}=0$ ). However, nine studies in these meta-analyses had been conducted in China, which, compared internationally, was less affected by the
SARS-CoV-2 pandemic [4]. In the subgroup analysis regarding the level of COVID-19 patient contact, we assigned studies to the subgroup 'high level of contact' if at least $50 \%$ of the sample had close contact to COVID-

\begin{tabular}{|c|c|c|c|c|c|c|c|c|c|}
\hline \multirow[b]{2}{*}{ Study } & \multirow[b]{2}{*}{ Total } & \multicolumn{2}{|c|}{ COVID-19 } & \multicolumn{3}{|c|}{ pre-COVID-19 } & Std. Mean Difference & \multirow{2}{*}{\multicolumn{2}{|c|}{$\begin{array}{l}\text { Std. Mean Difference } \\
\text { IV, Random, } 95 \% \mathrm{CI}\end{array}$}} \\
\hline & & Mean & SD & Total & Mean & SD & IV, Random, 95\% Cl & & \\
\hline Bauerle 2020 (GAD-7) & 15037 & 5.26 & 4.9100 & 5030 & 2.95 & 3.4100 & $0.50[0.47,0.54]$ & & \\
\hline Chang 2020 (GAD-7) & 3881 & 3.03 & 3.1250 & 1096 & 2.80 & 2.9000 & $0.07[0.01,0.14]$ & & 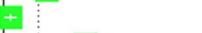 \\
\hline Germani 2020 (STAI-Y) & 1011 & 48.60 & 12.7000 & 2983 & 37.38 & 11.5100 & $0.95[0.87,1.02]$ & & + \\
\hline Gonzalez-Sanguino 2020 (GAD-2) & 3480 & 1.79 & 1.6300 & 110 & 1.21 & 1.3900 & $0.36[0.17,0.55]$ & & $\div$ \\
\hline Guo 2020 (non-infected controls; GAD-7) & 103 & 2.58 & 3.9050 & 1096 & 2.80 & 2.9000 & $-0.07[-0.27,0.13]$ & & \\
\hline Hao F 2020 (controls; DASS-21 anxiety) & 109 & 1.50 & 2.7000 & 13208 & 2.48 & 2.5900 & $-0.38[-0.57,-0.19]$ & $\div$ & \\
\hline lasevoli 2020 (caregivers; GAD-7) & 51 & 5.30 & 4.1000 & 339 & 3.90 & 4.4900 & $0.31[0.02,0.61]$ & & \\
\hline Iasevoli 2020 (controls, GAD-7) & 205 & 5.50 & 4.3000 & 5030 & 2.95 & 3.4100 & $0.74[0.60,0.88]$ & & + \\
\hline Lei 2020 (SAS) & 1593 & 36.47 & 9.1500 & 1158 & 29.78 & 0.4600 & $0.96[0.88,1.04]$ & & + \\
\hline Ma 2020 (DASS-21 anxiety) & 123 & 4.40 & 2.3000 & 1815 & 4.10 & 3.5000 & $0.09[-0.10,0.27]$ & & + \\
\hline Mazza 2020 (DASS-21 anxiety) & 2766 & 2.89 & 3.6900 & 417 & 2.40 & 2.6000 & $0.14[0.03,0.24]$ & & + \\
\hline McKay 2020 (DASS-21 anxiety) & 908 & 3.93 & 3.1700 & 13208 & 2.48 & 2.5900 & $0.55[0.48,0.62]$ & & + \\
\hline Odriozola-Gonzalez 2020 (DASS-21 anxiety) & 2530 & 3.34 & 3.8700 & 1055 & 4.84 & 5.7500 & $-0.33[-0.41,-0.26]$ & + & \\
\hline Ozdin 2020 (HADS anxiety) & 343 & 6.80 & 4.2000 & 1102 & 8.84 & 3.6200 & $-0.54[-0.66,-0.42]$ & + & \\
\hline Satici 2020 (DASS-21 anxiety) & 1304 & 2.73 & 3.1700 & 250 & 2.41 & 2.5300 & $0.10[-0.03,0.24]$ & & + \\
\hline Soraci 2020 (HADS anxiety) & 249 & 7.20 & 4.1000 & 21644 & 6.80 & 4.2000 & $0.10[-0.03,0.22]$ & & - \\
\hline Tan W 2020 (DASS-21 anxiety) & 673 & 1.60 & 3.7000 & 13208 & 2.48 & 2.5900 & $-0.33[-0.41,-0.25]$ & + & \\
\hline Tian 2020 (SCL-90 anxiety) & 1060 & 1.91 & 0.7100 & 1388 & 1.39 & 0.4300 & $0.91[0.83,1.00]$ & & + \\
\hline Tsipropoulou 2020 (GAD-7) & 2970 & 13.24 & 4.6600 & 2271 & 5.20 & 3.9820 & $1.84[1.77,1.90]$ & & + \\
\hline Tull 2020 (DASS-21 anxiety) & 500 & 5.30 & 8.0500 & 499 & 3.99 & 6.2700 & $0.18[0.06,0.31]$ & & + \\
\hline Wang C 2020b (1st survey; DASS-21 anxiety) & 1210 & 6.20 & 6.6000 & 13208 & 2.48 & 2.5900 & $1.19[1.13,1.25]$ & & + \\
\hline Wang C 2020b (2nd survey; DASS-21 anxiety) & 861 & 6.20 & 6.9000 & 13208 & 2.48 & 2.5900 & $1.23[1.16,1.30]$ & & \\
\hline Wang Y 2020 (SAS) & 600 & 36.92 & 7.3300 & 1158 & 29.78 & 0.4600 & $1.66[1.55,1.77]$ & & + \\
\hline Yuan R 2020 (child hosp. pand.; HADS anxiety) & 50 & 7.02 & 3.0100 & 8284 & 5.50 & 3.8000 & $0.40[0.12,0.68]$ & & $\div$ \\
\hline Yuan R 2020 (child not hosp. pand.; HADS anxiety) & 50 & 3.62 & 2.1000 & 8284 & 5.50 & 3.8000 & $-0.50[-0.77,-0.22]$ & $\longrightarrow$ & \\
\hline Zhou SJ 2020 (GAD-7) & 8079 & 3.94 & 4.3740 & 1096 & 2.80 & 2.9000 & $0.27[0.21,0.33]$ & & + \\
\hline Total & 49746 & & & 132145 & & & $0.40[0.15,0.65]$ & & \\
\hline Prediction interval & & & & & & & 5071671 & & \\
\hline Heterogeneity: $\mathrm{Tau}^{2}=0.40 ; \mathrm{Chi}^{2}=4995.25, \mathrm{df}=25(\mathrm{P}=$ & $0) ; I^{2}=S$ & $99 \%$ & & & & & & \begin{tabular}{l|l|l|l|l}
1 & 1
\end{tabular} & 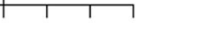 \\
\hline Test for overall effect: $Z=3.15(P<0.01)$ & & & & & & & & $\begin{array}{l}-1.5-1-0.5 \\
\text { reduced anxiety }\end{array}$ & $\begin{array}{llll}0 & 0.5 & 1 & 1.5 \\
\text { increased anxiety }\end{array}$ \\
\hline \multicolumn{10}{|c|}{$\begin{array}{l}\text { Abbreviations: CI, confidence interval; df, degrees of freedom; } \mathrm{I}^{2} \text {, indicator of statistical heterogeneity; } \mathrm{P}, \mathrm{p} \text { value; } \mathrm{SD} \text {, standard deviation; Std., } \\
\text { standardized; Tau }{ }^{2} \text {, indicator of statistical heterogeneity; Total, the number of participants; } \mathrm{Z}, \mathrm{z} \text { value; } \mathrm{Chi}^{2}, \mathrm{Chi}^{2} \text { test for heterogeneity. } \\
{ }^{\mathrm{a}} \text { Horizontal lines indicate the } 95 \% \mathrm{CI} \text { of each study; diamond, the pooled estimate with } 95 \% \mathrm{CI} \text {; multilevel meta-analysis. }\end{array}$} \\
\hline \multicolumn{10}{|c|}{ Fig. 2 Forest plot main analysis, general population, anxiety } \\
\hline
\end{tabular}




\section{Study}

Bauerle 2020 (PHQ-2)

Chang 2020 (PHQ-9)

Gonzalez-Sanguino 2020 (PHQ-2)

Guo 2020 (non-infected controls; PHQ-9)

Hao F 2020 (controls; DASS-21 depression)

lasevoli 2020 (caregivers; PHQ-9)

lasevoli 2020 (controls; PHQ-9)

Lei 2020 (SDS)

Ma 2020 (DASS-21 depression)

Mazza 2020 (DASS-21 depression)

McKay 2020 (DASS-21 depression)

Odriozola-Gonzalez 2020 (DASS-21 depression)

Olagoke 2020 (PHQ-2)

Ozdin 2020 (HADS depression)

Sakib 2020 (PHQ-9)

Satici 2020 (DASS-21 depression)

Soraci 2020 (HADS depression)

Tan W 2020 (DASS-21 depression)

Tian 2020 (SCL-90 depression)

Tsipropoulou (Greece, GAD-7)

Tull (USA, DASS-21)

Voitsidis 2020 (PHQ-2)

Wang C 2020b (1st survey: DASS-21 depression)

Wang C 2020b (2nd survey; DASS-21 depression)

Wang Y 2020 (SDS)

Yuan R 2020 (child hospit. pand.; HADS depression)

Yuan R 2020 (child not hosp. pand.; HADS depressi)

Zhou SJ 2020 (PHQ-9)

Total

Prediction interval

Heterogeneity: $\mathrm{Tau}^{2}=1.79 ; \mathrm{Chi}^{2}=16430.33, \mathrm{df}=27(\mathrm{P}=0) ; \mathrm{I}^{2}=100 \%$

\section{COVID-19}

Total Mean SD

$\begin{array}{lll}15037 & 1.14 & 1.5300\end{array}$

$\begin{array}{lll}3881 & 245 & 3.5200\end{array}$

$\begin{array}{lll}3480 & 1.60 & 1.5000\end{array}$

$\begin{array}{lll}103 & 3.46 & 4.5410\end{array}$

$\begin{array}{lll}109 & 2.20 & 3.5000\end{array}$

$\begin{array}{lll}51 & 4.20 & 3.2000\end{array}$

$\begin{array}{lll}205 & 6.20 & 4.5000\end{array}$

$1593 \quad 37.14 \quad 11.3700$

$\begin{array}{lll}123 & 4.20 & 23000\end{array}$

$\begin{array}{lll}2766 & 5.34 & 4.8100\end{array}$

$\begin{array}{lll}908 & 2.77 & 3.2100\end{array}$

$\begin{array}{lll}2530 & 5.52 & 4.9200\end{array}$

$\begin{array}{lll}501 & 1.92 & 0.9300\end{array}$

$\begin{array}{lll}343 & 6.70 & 4.2000\end{array}$

$\begin{array}{lll}8550 & 7.43 & 5.0600\end{array}$

$1304 \quad 3.86 \quad 4.1900$

$\begin{array}{lll}249 & 8.90 & 3.6000\end{array}$

$\begin{array}{lll}673 & 2.10 & 4.5000\end{array}$

$\begin{array}{lll}1060 & 1.96 & 0.7000\end{array}$

$2970 \quad 14.70 \quad 4.5100$

$\begin{array}{lll}500 & 7.51 & 9.0000\end{array}$

$\begin{array}{lll}2427 & 4.17 & 1.4200\end{array}$

$\begin{array}{lll}1210 & 6.30 & 7.2000\end{array}$

$\begin{array}{lll}861 & 6.40 & 7.4000\end{array}$

$600 \quad 40.50 \quad 11.3100$

$\begin{array}{lll}50 & 7.72 & 2.8100\end{array}$

$\begin{array}{lll}50 & 4.54 & 25600\end{array}$

$8079 \quad 5.13 \quad 5.6090$

60213

Test for overall effect: $Z=2.20(P=0.03)$

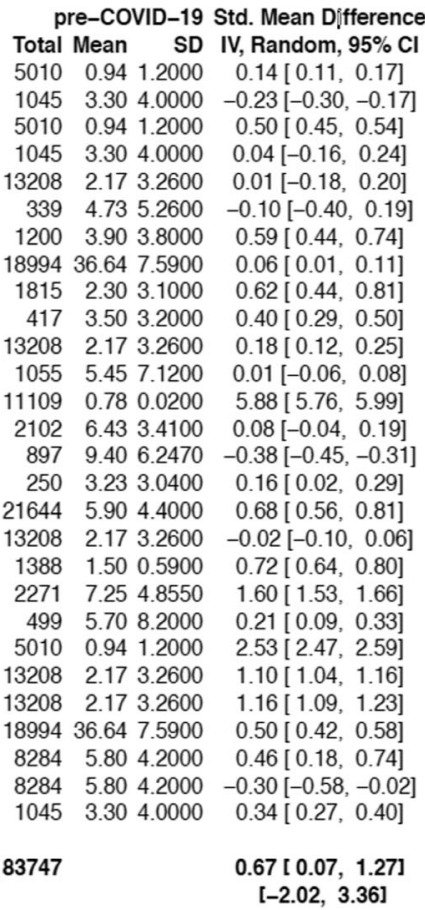

$[-2.02,3.36]$

183747

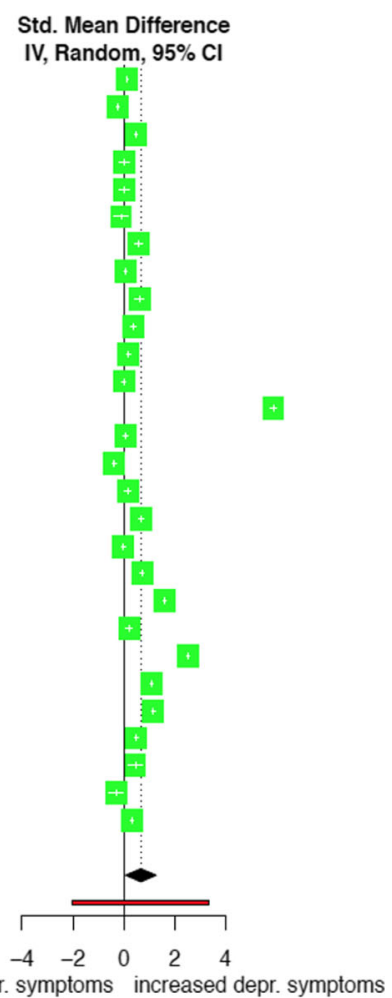

Abbreviations: CI, confidence interval; df, degrees of freedom; $\mathrm{I}^{2}$, indicator of statistical heterogeneity; $\mathrm{P}, \mathrm{p}$ value; SD, standard deviation; Std., standardized; $\mathrm{Tau}^{2}$, indicator of statistical heterogeneity; Total, the number of participants; $\mathrm{Z}, \mathrm{z}$ value; $\mathrm{Chi}^{2}$, Chi ${ }^{2}$ test for heterogeneity.

${ }^{a}$ Horizontal lines indicate the $95 \%$ CI of each study; diamond, the pooled estimate with $95 \%$ CI; multilevel meta-analysis.

Fig. 3 Forest plot main analysis, general population, depression

19 patients (ie, 'frontline healthcare workers'). However, the nature of contact was insufficiently described in the included studies.

Strengths of this review compared with previous publications include the systematic search for comparative prepandemic data for inclusion in pairwise metaanalyses, the stepwise selection of prepandemic studies to ensure best available comparability, and the population-specific analysis of risk and protective factors. One limitation refers to the search methods for pandemic studies (eg, no preprints; no reference lists of reviews) and comparative data (eg, subgroups in general population only partially searched). We had no restrictions regarding the publication format except for the exclusion of preprints which might be viewed as limitation. This restriction might have affected the evidence found in this review compared to others (eg, Cochrane reviews) where preprint articles are included.

The large between-study heterogeneity, a problem shared by previous meta-analyses [20, 24, 32, 33], could not be fully explained by subgroup analyses. This heterogeneity probably resulted from differences between the pandemic studies (eg, countries, sociocultural differences in the perception of mental burden, pandemic outbreak severity, subpopulations, outcome measures) and variability between the comparative studies (eg, study design, outcome measures), respectively. Among the pandemic studies, especially the specific outcome measures used were an important source of heterogeneity. Furthermore, the pandemic and comparative data were heterogeneous (eg, country, population), which could be partially captured by our selfdeveloped tool for the level of comparability and was controlled for by the corresponding sensitivity analysis. We cannot preclude that moderators of effects are present that we, though our best efforts, did not identify and therefore could not control for. Besides, comparative studies with larger sample sizes were preferred, leading to small 95\% CIs and a lack of CI overlap with pandemic study findings. Despite the comprehensiveness of this review compared to previous publications, the small number of studies in certain subgroups potentially limited the statistical power (eg, surveys including students).

Apart from specific outcome measures, less recent comparative data, and homogenous sample sizes, the subgroup analyses indicated no consistent determinants of heterogeneity. An elevated level of depression based on the assessment with the PHQ and SDS might - at least for the PHQ-9 - be explained by the high 
Table 4 Results of subgroup analyses for those populations and outcomes with at least $k=4$ studies in main analysis

\begin{tabular}{|c|c|c|c|c|c|}
\hline $\begin{array}{l}\text { Subgroup analysis } \\
\text { (subgroups) }\end{array}$ & Outcome & $\begin{array}{l}\text { Test for subgroup } \\
\text { differences }^{\mathrm{a}}\end{array}$ & Population & 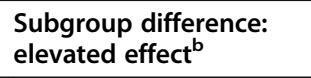 & $\begin{array}{l}\text { Subgroup difference: } \\
\text { reduced effect }^{\mathrm{b}}\end{array}$ \\
\hline \multicolumn{6}{|c|}{ Population characteristics (main studies) } \\
\hline \multirow{8}{*}{$\begin{array}{l}\text { Age } \\
\cdot 30 \text { years } \\
\cdot>30 \leq 35 \text { years } \\
\cdot>35 \leq 40 \text { years } \\
\cdot>40 \leq 45 \text { years } \\
\cdot \text { - multiple age groups } \\
\cdot \text { age not specified }\end{array}$} & Anxiety & $\mathrm{Chi}^{2}=9.5, \mathrm{df}=5(p=.09)$ & GP & / & / \\
\hline & Depression & $\begin{array}{l}\text { Chi }^{2}=29.3, \mathrm{df}=5 \\
(p<.001)\end{array}$ & GP & $\leq 30$ years; $>40 \leq 45$ years & / \\
\hline & Stress & $\begin{array}{l}C h i^{2}=1043.3, \mathrm{df}=4 \\
(p<.001)\end{array}$ & GP & / & $>40 \leq 45$ years \\
\hline & Anxiety & $\mathrm{Chi}^{2}=8.7, \mathrm{df}=4(p=.07)$ & $\mathrm{HCW}$ & / & / \\
\hline & Depression & $\mathrm{Chi}^{2}=2.2, \mathrm{df}=1(p=.14)$ & $\mathrm{HCW}$ & / & / \\
\hline & Sleep & $\mathrm{Chi}^{2}=0.3, \mathrm{df}=1 \quad(p=.57)$ & $\mathrm{HCW}$ & / & / \\
\hline & Anxiety & $\begin{array}{l}\mathrm{Chi}^{2}=17.14, \mathrm{df}=4 \\
(p=.002)\end{array}$ & $P$ & $>40 \leq 45$ years & \\
\hline & Depression & $\mathrm{Chi}^{2}=3.74, \mathrm{df}=4(p=.44)$ & $P$ & / & / \\
\hline \multirow{3}{*}{$\begin{array}{l}\text { Stressor exposure } \\
\text { - General population } \\
\text { - Students } \\
\text { - Others } \\
\text { - Special exposure }\end{array}$} & Anxiety & $\mathrm{Chi}^{2}=2.8, \mathrm{df}=3(p=.42)$ & GP & / & / \\
\hline & Depression & $\mathrm{Chi}^{2}=1.9, \mathrm{df}=3(p=.60)$ & GP & / & / \\
\hline & Stress & $\mathrm{Chi}^{2}=0.12, \mathrm{df}=3(p=.99)$ & GP & / & / \\
\hline \multirow{3}{*}{$\begin{array}{l}\text { Covid-19 patient contact } \\
\text { - Low contact risk } \\
\text { - High contact risk }\end{array}$} & Anxiety & $\mathrm{Chi}^{2}=0, \mathrm{df}=1 \quad(p=.95)$ & $\mathrm{HCW}$ & / & / \\
\hline & Depression & $\mathrm{Chi}^{2}=1.0, \mathrm{df}=1(p=.31)$ & $\mathrm{HCW}$ & / & / \\
\hline & Sleep & $\mathrm{Chi}^{2}=0.2, \mathrm{df}=1 \quad(p=.69)$ & $\mathrm{HCW}$ & / & / \\
\hline \multirow{2}{*}{$\begin{array}{l}\text { Subgroup of patients } \\
\text { - COVID-19 patients } \\
\text { - Pregnant women }\end{array}$} & Anxiety & $\mathrm{Chi}^{2}=0.3, \mathrm{df}=2(p=.88)$ & $P$ & / & / \\
\hline & Depression & $C h i^{2}=1.3, d f=2(p=.51)$ & $P$ & / & / \\
\hline
\end{tabular}

- Psychiatric patients

\section{Pandemic study characteristics}

\section{Survey start ${ }^{\mathrm{C}}$ \\ $\cdot \leq 4$ weeks \\ $\cdot>4 \leq 6$ weeks \\ $\cdot>6 \leq 8$ weeks \\ $\cdot>8$ weeks \\ - not specified}

Study conduction China

- China

- Non-China

Outcome measure

- AIS

- BDI

- DASS-21

- EDPS

- EPDS-3A

- GAD-2; GAD-7

- HADS

\begin{tabular}{|c|c|c|c|}
\hline Anxiety & $\mathrm{Chi}^{2}=3.55, \mathrm{df}=4(p=.47)$ & GP & / \\
\hline Depression & $\begin{array}{l}C h i^{2}=10.15, \mathrm{df}=4 \\
(p=.04)\end{array}$ & GP & $>8$ weeks \\
\hline Stress & $\mathrm{Chi}^{2}=0.31, \mathrm{df}=4(p=.99)$ & GP & / \\
\hline Anxiety & $\mathrm{Chi}^{2}=7.91, \mathrm{df}=4(p=.10)$ & HCW & / \\
\hline Depression & $C h i^{2}=0.95, d f=2(p=.62)$ & HCW & / \\
\hline Sleep & $\mathrm{Chi}^{2}=4.21, \mathrm{df}=2(p=.12)$ & $\mathrm{HCW}$ & / \\
\hline Anxiety & $\mathrm{Chi}^{2}=4.58, \mathrm{df}=2(p=.10)$ & $P$ & / \\
\hline Depression & $\mathrm{Chi}^{2}=3.08, \mathrm{df}=3(p=.38)$ & $P$ & / \\
\hline Anxiety & $C h i^{2}=0.10, d f=1(p=.75)$ & GP & / \\
\hline Depression & $\mathrm{Chi}^{2}=0.60, \mathrm{df}=1 \quad(p=.44)$ & GP & / \\
\hline Stress & $\mathrm{Chi}^{2}=0.10, \mathrm{df}=1 \quad(p=.76)$ & GP & / \\
\hline Anxiety & $\mathrm{Chi}^{2}=2.84, \mathrm{df}=1 \quad(p=.09)$ & $\mathrm{HCW}$ & / \\
\hline Depression & $\mathrm{Chi}^{2}=0.08, \mathrm{df}=1 \quad(p=.78)$ & HCW & / \\
\hline Sleep & $\mathrm{Chi}^{2}=0.32, \mathrm{df}=1 \quad(p=.57)$ & HCW & / \\
\hline Anxiety & $\mathrm{Chi}^{2}=3.35, \mathrm{df}=1 \quad(p=.07)$ & $P$ & / \\
\hline Depression & $\mathrm{Chi}^{2}=0.62, \mathrm{df}=1 \quad(p=.43)$ & $P$ & / \\
\hline Anxiety & $\mathrm{Chi}^{2}=10.7, \mathrm{df}=6(p=.10)$ & GP & / \\
\hline Depression & $\begin{array}{l}\mathrm{Chi}^{2}=11.46, \mathrm{df}=5 \\
(p=.04)\end{array}$ & GP & PHQ-2 \\
\hline Stress & $\mathrm{Chi}^{2}=0.16, \mathrm{df}=1 \quad(p=.69)$ & GP & / \\
\hline Anxiety & $C h i^{2}=2.80, d f=4(p=.59)$ & HCW & l \\
\hline Depression & $\mathrm{Chi}^{2}=2.91, \mathrm{df}=3(p=.41)$ & $\mathrm{HCW}$ & / \\
\hline
\end{tabular}


Table 4 Results of subgroup analyses for those populations and outcomes with at least $k=4$ studies in main analysis (Continued)

\begin{tabular}{|c|c|c|c|c|c|}
\hline $\begin{array}{l}\text { Subgroup analysis } \\
\text { (subgroups) }\end{array}$ & Outcome & $\begin{array}{l}\text { Test for subgroup } \\
\text { differences }^{\mathrm{a}}\end{array}$ & Population & $\begin{array}{l}\text { Subgroup difference: } \\
\text { elevated effect }^{\mathrm{b}}\end{array}$ & $\begin{array}{l}\text { Subgroup difference: } \\
\text { reduced effect }^{\text {b }}\end{array}$ \\
\hline \multirow{3}{*}{$\begin{array}{l}\text { - HAMA } \\
\text { - HAMD } \\
\text { - ISI } \\
\text { - PHQ-2; PHQ-9 } \\
\text { - PSQI } \\
\text { - PSS } \\
\text { - SAS } \\
\text { - SDS } \\
\text { - SCL-90 } \\
\text { - STAI-Y }\end{array}$} & Sleep & $\mathrm{Chi}^{2}=0.32, \mathrm{df}=1 \quad(p=.57)$ & $\mathrm{HCW}$ & I & / \\
\hline & Anxiety & $\mathrm{Chi}^{2}=1.18, \mathrm{df}=4(p=.88)$ & P & / & / \\
\hline & Depression & $\begin{array}{l}\text { Chi }^{2}=16.95, \mathrm{df}=5 \\
(p=.005)\end{array}$ & $P$ & SDS; PHQ-9 & / \\
\hline \multirow{8}{*}{$\begin{array}{l}\text { Sample size } \\
\cdot<1000 \\
\cdot \geq 1000\end{array}$} & Anxiety & $\mathrm{Chi}^{2}=1.86, \mathrm{df}=1(p=.17)$ & GP & / & / \\
\hline & Depression & $\mathrm{Chi}^{2}=0.03, \mathrm{df}=1 \quad(p=.86)$ & GP & / & / \\
\hline & Stress & $\mathrm{Chi}^{2}=2.31, \mathrm{df}=1 \quad(p=.13)$ & GP & / & / \\
\hline & Anxiety & $\mathrm{Chi}^{2}=2.83, \mathrm{df}=1 \quad(p=.09)$ & HCW & / & / \\
\hline & Depression & $\mathrm{Chi}^{2}=0, \mathrm{df}=1(p=.96)$ & $\mathrm{HCW}$ & / & / \\
\hline & Sleep & not possible & HCW & / & / \\
\hline & Anxiety & $\mathrm{Chi}^{2}=3.60, \mathrm{df}=1 \quad(p=.06)$ & P & / & / \\
\hline & Depression & $\mathrm{Chi}^{2}=0.09, \mathrm{df}=1 \quad(p=.77)$ & $P$ & / & / \\
\hline \multicolumn{6}{|c|}{ Comparative study characteristics } \\
\hline \multirow{8}{*}{$\begin{array}{l}\text { Sample size } \\
\cdot \leq 500 \\
\cdot>1000 \leq 5000 \\
\cdot>5000 \leq 10,000 \\
\cdot>10,000\end{array}$} & Anxiety & $C h i^{2}=0.9, \mathrm{df}=3(p=.83)$ & GP & / & / \\
\hline & Depression & $C h i^{2}=3.5, d f=4(p=.48)$ & GP & / & / \\
\hline & Stress & $C h i^{2}=8.6, d f=3(p=.03)$ & GP & / & $>5000 \leq 10,000$ participants \\
\hline & Anxiety & $\mathrm{Chi}^{2}=9.93, \mathrm{df}=3(p=.02)$ & $\mathrm{HCW}$ & $>5000 \leq 10,000$ participants & \\
\hline & Depression & $\mathrm{Chi}^{2}=4.3, \mathrm{df}=2(p=.12)$ & HCW & / & / \\
\hline & Sleep & $C h i^{2}=0.3, d f=1(p=.57)$ & HCW & / & / \\
\hline & Anxiety & $C h i^{2}=0.1, d f=2(p=.97)$ & $P$ & / & / \\
\hline & Depression & $C h i^{2}=3.9, d f=2(p=.14)$ & $P$ & I & / \\
\hline \multirow{8}{*}{$\begin{array}{l}\text { Publication year } \\
\cdot \leq 1 \text { year ago } \\
\cdot \leq 2 \text { years ago } \\
\cdot>2 \leq 5 \text { years ago } \\
\cdot>5 \leq 10 \text { years ago } \\
\cdot>10 \text { years ago }\end{array}$} & Anxiety & $C h i^{2}=8.0, d f=5(p=.16)$ & GP & / & / \\
\hline & Depression & $\mathrm{Chi}^{2}=12.4, \mathrm{df}=5(p=.03)$ & GP & $>10$ years ago & / \\
\hline & Stress & $\mathrm{Chi}^{2}=11.6, \mathrm{df}=4(p=.02)$ & GP & / & $\leq 1$ year ago \\
\hline & Anxiety & $\begin{array}{l}\mathrm{Chi}^{2}=14.5, \mathrm{df}=3 \\
(p=.002)\end{array}$ & HCW & $>10$ years ago & $\leq 2$ years ago \\
\hline & Depression & $C h i^{2}=4.6, d f=1(p=.03)$ & HCW & / & $\leq 2$ years ago \\
\hline & Sleep & not possible & $\mathrm{HCW}$ & / & / \\
\hline & Anxiety & $C h i^{2}=0.1, d f=2(p=.94)$ & $P$ & / & / \\
\hline & Depression & $\begin{array}{l}C h i^{2}=17.0, d f=5 \\
(p=.005)\end{array}$ & $P$ & $\leq 1$ year ago; $>5 \leq 10$ years ago & / \\
\hline \multicolumn{6}{|c|}{ Pandemic and comparative study characteristics } \\
\hline \multirow{5}{*}{$\begin{array}{l}\text { Relationship samples } \\
\text { sizes }^{d} \\
\text { - Ratio } \geq 2 \\
\text { - Ratio } \geq 0.5<2 \\
\text { - Ratio } \geq 0.1<0.5 \\
\text { - Ratio }<0.1\end{array}$} & Anxiety & $C h i^{2}=10.0, d f=3(p=.02)$ & $\mathrm{GP}$ & Ratio $\geq 0.5<2$ & / \\
\hline & Depression & $\mathrm{Chi}^{2}=4.8, \mathrm{df}=3(p=.19)$ & GP & / & / \\
\hline & Stress & $\mathrm{Chi}^{2}=0.4, \mathrm{df}=2(p=.84)$ & GP & / & / \\
\hline & Anxiety & $C h i^{2}=4.2, d f=2(p=.12)$ & HCW & / & / \\
\hline & Depression & $\mathrm{Chi}^{2}=3.8, \mathrm{df}=2(p=.15)$ & $\mathrm{HCW}$ & / & / \\
\hline
\end{tabular}


Table 4 Results of subgroup analyses for those populations and outcomes with at least $k=4$ studies in main analysis (Continued)

\begin{tabular}{|c|c|c|c|c|c|}
\hline $\begin{array}{l}\text { Subgroup analysis } \\
\text { (subgroups) }\end{array}$ & Outcome & $\begin{array}{l}\text { Test for subgroup } \\
\text { differences }^{\mathrm{a}}\end{array}$ & Population & 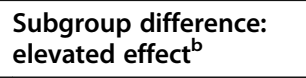 & $\begin{array}{l}\text { Subgroup difference: } \\
\text { reduced effect }^{b}\end{array}$ \\
\hline & Sleep & $\mathrm{Chi}^{2}=0.32, \mathrm{df}=1 \quad(p=.57)$ & $\mathrm{HCW}$ & 1 & / \\
\hline & Anxiety & $\begin{array}{l}\mathrm{Chi}^{2}=17.7, \mathrm{df}=3 \\
(p<.001)\end{array}$ & $P$ & Ratio $\geq 0.5<2$; Ratio $<0.1$ & / \\
\hline & Depression & $\mathrm{Chi}^{2}=3.0, \mathrm{df}=3(p=.39)$ & $P$ & / & / \\
\hline
\end{tabular}

Abbreviations: AIS Athens Insomnia Scale, BDI Beck Depression Inventory, DASS-21 Depression Anxiety Stress Scale-21, $d f$ degrees of freedom, EPDS Edinburgh Postnatal Depression Scale, EPDS-3A Edinburgh Postnatal Depression Scale-Anxiety subscale, GAD Generalized Anxiety Disorder Scale, GP general population, HADS Hospital Anxiety and Depression Scale, HAMA Hamilton Anxiety Rating Scale, HAMD Hamilton Depression Rating Scale, HCW healthcare workers, $p$ p value, $P$ patients, PHQ Patient Health Questionnaire, PSS Perceived Stress Scale, SAS Self-Rating Anxiety Scale, SCL-90 Symptom Checklist-90, SDS Zung Self-Rating Depression Scale, STAI-Y, State Trait Anxiety Inventory-Y

${ }^{\text {a }} \mathrm{Chi}^{2}=$ test for subgroup differences

b ordered by size of effect estimate (SMD)

' since first COVID-19 cases in the respective country or, in case of China, since January 20, 2020

${ }^{d}$ ratio of sample size in pandemic study vs comparative study

sensitivity to change of this instrument and its usefulness to monitor treatment outcomes [200, 201]. Given the increased mental burden if pandemic studies were compared to older prepandemic data, cohort effects cannot be excluded.

Discrepancies between subgroup analyses and the narrative synthesis of risk and protective factors (eg, COVID-19 patient contact) might be due to methodological differences. Because of the primary use of screening but not diagnostic tools to determine mental burden in the included pandemic studies, this review does not allow any conclusions concerning a putative increase of diagnoses of mental disorders during the early phase of the SARS-CoV-2 pandemic. Consistent with the synthesis of risk factors, the meta-analyses partly showed an increased level of mental symptoms in young and middleaged groups, in line with previous studies [12]. However, more studies including elderly would be needed to clearly investigate age differences, and whether the pandemic works as a 'burning lens' for the already increased mental burden in young people [202]. Finally, given the pandemic timeline, the evidence is substantially based

Table 5 Risk and protective factors in three populations (mostly frequently reported factors)

\begin{tabular}{|c|c|c|}
\hline & Risk factors $^{\mathrm{a}}$ & Protective factors $^{b}$ \\
\hline $\begin{array}{l}\text { General } \\
\text { population }\end{array}$ & $\begin{array}{l}\text { - Mental disorder/or symptoms }[44,49,51,52,58,64,69,74,78,82 \text {, } \\
83,116,132,135]^{1} \text { ) } \\
\text { - Worries about relatives or oneself }[51,57,64,66,74,75,80,89,82 \text {, } \\
83]^{1}[48]^{1,2} \\
\text { - Being female }[49,52,63,66,69,72,74,79,82,83,93]^{1}[\mathrm{vs} 1 \times \text { xeing } \\
\text { male] } \\
\text { - Previous (chronic) medical disease }[52,55,63,64,69,85,135]^{1} \\
\text { - Being a student }[52,57,60,72,146]^{1} \\
\text { - Personal/social worries about COVID-19 }[51,85,86,145]^{1}[48]^{1,2} \\
\text { - Physical symptoms }[52,66,80,85,132]^{1} \\
\text { - Reduced perceived health }[50,57,80,85,132]^{1} \\
\text { - No current relationship }[57,80,81,146]^{1} \\
\text { - Current local outbreak severity }[57,88,93,141]^{1} \\
\text { - History of stressful situations }[52,58,63,147]^{1} \\
\text { - Vulnerability to COVID-19 }[53,85,146]^{1} \\
\text { - Health profession }[66,81,141]^{1} \\
\text { - Own or close person's quarantine }[57,62,85]^{1}\end{array}$ & $\begin{array}{l}\text { - Older age }[49,52,63,65,66,79,91,140,147]^{1} \\
\text { - Good economic situation }[52,79,88,140,146]^{1}[48]^{1,2} \\
\text { - Satisfaction with/level of information on COVID-19 }[45, \\
49,52,85,88,93]^{1} \\
\text { - Not being single }[66,80,88,86]^{1} \\
\text { - Higher education }[50,52,66,146]^{1} \\
\text { - Social support }[52,140]^{1}[48]^{1,2} \\
\text { - Being male }[54,65,85]^{1}\end{array}$ \\
\hline $\begin{array}{l}\text { Healthcare } \\
\text { workers }\end{array}$ & $\begin{array}{l}\text { - Mental disorder/or symptoms }[97,115,116,119,122]^{1} \\
\text { - Being female }[98,110,119,121]^{1} \\
\text { - Concern about infection with COVID-19 }[103,109,120,121]^{1} \\
\text { - Exposure to COVID-19 patients }[94,110,115,119]^{1} \\
\text { - Current local COVID-19 severity }[94,118,141,107]^{1}\end{array}$ & - Older age $[98,110]^{1}$ \\
\hline Patients & $\begin{array}{l}\text { - (Suspected) COVID-19 }[42,131,143]^{1} \\
\text { - Inflammatory markers in blood }[42,131]^{1} \\
\text { - Physical symptoms }[132]^{1}\end{array}$ & $\begin{array}{l}\text { - Higher education }[127]^{1,3} \\
\text { - Good economic situation [127] } \\
\text { - Higher lymphocyte ratio in blood [42] } \\
\text { - Concomitant medical diseases [135] }\end{array}$ \\
\hline
\end{tabular}

\footnotetext{
a most frequently reported risk factors: general population: factor was reported as statistically significant risk factor in at least $\mathrm{k}=3$ studies; healthcare workers: factor reported in at least $k=4$ studies; patients: factor reported in at least $k=2$ studies

${ }^{\mathrm{b}}$ most frequently reported protective factors: general population: factor was reported as statistically significant protective factor in at least $\mathrm{k}=3$ studies; healthcare workers: factor reported in at least $k=2$ studies (limited number of studies reporting protective factors in this group); patients: factor reported in $k=1$ study (limited number of studies reporting protective factors in this group)
} 
on Chinese studies thus potentially limiting the transferability of findings to other contexts.

Further research in other countries (eg, USA), that started later on during the pandemic, could change the findings. The latter is also supported by the wide prediction intervals identified in this review, which indicate uncertainty in our conclusions about whether the pandemic and related stressors do affect mental health [203].

The review has several implications for research and practice. There is an urgent need for representative surveys, in order to allow fair comparisons between the mental burden caused by SARS-CoV-2 in different countries and to examine other risk and protective factors (eg, cultural context). Representative surveys in the general population might also serve to identify specific subgroups at risk for which further studies would be needed. From a public mental health perspective, a stronger focus on (psychosocial) protective factors for mental health would be desirable to derive appropriate contents for preventive measures (eg, pandemic preparedness plans) or health-promoting interventions (eg, resilience training) prior to, during, and after a pandemic [199]. By further investigating the mental health impact of specific stressors - in line with Brooks and colleagues [13] - researchers and practitioners might gain further knowledge about when (eg, in pandemic timeline) and for whom (eg, after exposure to which stressors) interventions should be implemented to buffer negative mental health effects of SARS-CoV-2.

\section{Conclusions}

In conclusion, compared with prepandemic data, this review shows different adverse mental health consequences of the early phase of the SARS-CoV-2 pandemic in the examined population groups in contrast to previous research, with healthcare workers being more resilient than expected. The quality of studies varies. High-quality, representative surveys in the general population and specific subpopulations, longitudinal studies, and further research efforts on protective factors are needed to better understand the psychological impacts of the SARS-CoV-2 pandemic and to help design effective preventive measures and interventions that are tailored to the needs of specific population groups.

\section{Supplementary Information}

The online version contains supplementary material available at https:/doi. org/10.1186/s12992-021-00670-y.

Additional file 1: Methods of the systematic review with meta-analyses. eTable 1. MOOSE Checklist. eTable 2. Differences between protocol and review. eMethods 1. Search strategies for SARS-CoV-2 ('pandemic') studies. eMethods 2. Search strategy for prepandemic comparative studies. eTable 3. Eligibility criteria for SARS-CoV-2 pandemic studies. eTable 4. Eligibility criteria for prepandemic comparative studies. eTable 5. Eligibility criteria for pairwise meta-analyses. eTable 6. Customized data extraction sheet. eTable 7. Modified quality assessment tool. eTable 8. Rating of comparability between pandemic and prepandemic comparative studies. eMethods 3. Further methodological details of this systematic review and meta-analyses.

Additional file 2: Results of the systematic review with meta-analyses. eTable 9. Details on number of included (pandemic and comparative) studies. eTable 10. Study characteristics of the prepandemic comparative studies. eTable 11. Cut-off values reported in included pandemic studies. eResults 1. Forest plots of main analyses. eTable 12. Quality assessment of included pandemic studies. eTable 13. Assessment of level of comparability between pandemic and prepandemic comparative studies. eResults 2. Forest plots of sensitivity analyses. eResults 3. Detailed results of subgroup analyses. eTable $\mathbf{1 4}$. Risk factors in the general population, healthcare workers, and patients. eTable 15. Protective factors in the general population, healthcare workers, and patients.

\section{Abbreviations}

COVID-19: Coronavirus disease 2019; LORA: Longitudinal Resilience Assessment; MOOSE: Meta-analyses Of Observational Studies in Epidemiology; NIH: National Institutes of Health; PHQ: Patient Health Questionnaire; PRISMA: Preferred Reporting Items for Systematic Reviews and Meta-Analysis; PROSPERO: International Prospective Register of Systematic Reviews; SARS-CoV(- 2): Severe Acute Respiratory Syndrome Coronavirus (2); SD: Standard deviation; SDS: Zung Self-Rating Depression Scale; SMD: Standardized mean difference

\section{Acknowledgements}

We appreciate the contributions of Prof Raffael Kalisch PhD to the discussion of the findings of our review.

\section{Authors' contributions}

AMK, NR, JSW, and KL designed the study. NR and LG, respectively, assessed study eligibility; KL was consulted in case of any disagreements. NR and LG extracted and analyzed data for Table 1, with KL being consulted in case of any disagreements. AMK, NR, and GS designed the statistical analyses. AMK and NR analyzed data for Tables 2, 3, 4 and 5 based on pairwise metaanalyses (including subgroup and sensitivity analyses) and the narrative synthesis of risk/protective factors. GS and HB reviewed the statistical analyses. $\mathrm{KL}$ monitored the review process. All authors contributed to the interpretation of the results, with special expertise provided in the field of resilience research (AMK, OT, KL), public health (MC, ER), and evidence-based medicine (CS, JJM). AMK wrote the first draft of the manuscript with input and subsequent edits by all authors. KL is the guarantor. All authors read and approved the final manuscript.

\section{Funding}

The CEOsys and the egePan project are funded under a scheme issued by the Network of University Medicine (Nationales Forschungsnetzwerk der Universitätsmedizin (NUM)) by the Federal Ministry of Education and Research of Germany (Bundesministerium für Bildung und Forschung (BMBF) Grant number 01KX2021). The Project RESPOND is funded by the EU RIA-call H2020-SC1-PHE-CORONAVIRUS-2020-2-RTD (Grant number 101016127). The funding body had no role in the design of the study and collection, analysis, and interpretation of data and in writing the manuscript. Open Access funding enabled and organized by Projekt DEAL.

\section{Availability of data and materials}

All data generated or analyzed during this study are included in this published article and its supplementary information files. Additional data (eg, detailed extracted data) are available from the corresponding author on request.

Ethics approval and consent to participate Not applicable. 


\section{Consent for publication}

Not applicable.

\section{Competing interests}

$L G, J S W$, and GS have no conflicts of interest to disclose. AMK, NR, OT, MC, $E R, H B, C S, J J M$, and KL report grants from the Federal Ministry of Education and Research (BMBF), Germany, during the conduct of the study. JJM reports grants from the Federal Ministry of Health (BMG), Germany, outside of the submitted work.

\section{Author details}

'Department of Psychiatry and Psychotherapy, University Medical Center of the Johannes Gutenberg University Mainz, Mainz, Germany. ${ }^{2}$ Leibniz Institute for Resilience Research (LIR), Mainz, Germany. ${ }^{3}$ Institute for Medical Information Processing, Biometry and Epidemiology, Chair of Public Health and Health Services Research, LMU Munich, Munich, Germany. ${ }^{4}$ Pettenkofer School of Public Health Munich, Munich, Germany. Institute of Medical Biometry and Statistics, Faculty of Medicine and Medical Center, University of Freiburg, Freiburg, Germany. ${ }^{6}$ Institute for Evidence in Medicine, Medical Center - University of Freiburg, Faculty of Medicine, University of Freiburg, Freiburg, Germany. ${ }^{7}$ Cochrane Germany, Cochrane Germany Foundation, Freiburg, Germany.

\section{Received: 2 December 2020 Accepted: 4 February 2021}

Published online: 29 March 2021

\section{References}

1. Lu R, Zhao X, Li J, et al. Genomic characterisation and epidemiology of 2019 novel coronavirus: implications for virus origins and receptor binding. Lancet. 2020;395(10224):565-74.

2. World Health Organization. Disease outbreak news. Novel coronavirus China. 2020. https://www.who.int/csr/don/12-january-2020-novel-corona virus-china/en/. Accessed 30 June 2020.

3. Sohrabi C, Alsafi Z, O'Neill N, et al. World Health Organization declares global emergency: a review of the 2019 novel coronavirus (COVID-19). Int J Surg. 2020:76:71-6.

4. World Health Organization. Coronavirus (COVID-19) Dashboard. 2020. https://who.sprinklr.com/. Accessed 28 Sept 2020.

5. BBC. Coronavirus: the world in lockdown in maps and charts. 2020. https:// www.bbc.com/news/world-52103747. Accessed 30 June 2020.

6. The Guardian. China's coronavirus lockdown strategy: brutal but effective. 2020. https://www.theguardian.com/world/2020/mar/19/chinas-coronaviruslockdown-strategy-brutal-but-effective. Accessed 7 May 2020

7. White House. The President's coronavirus guidelines for America. 2020. Accessed 30 June 2020.

8. World Health Organization. Pulse survey on continuity of essential health services during the COVID-19 pandemic: interim report. 2020. https://www. who.int/publications/i/item/WHO-2019-nCoV-EHS_continuity-survey-2020.1. Accessed 4 Nov 2020

9. Phua J, Weng $L$, Ling $L$, et al. Intensive care management of coronavirus disease 2019 (COVID-19): challenges and recommendations. Lancet Respir Med. 2020;8(5):506-17.

10. Pak A, Adegboye OA, Adekunle Al, Rahman KM, McBryde ES, Eisen DP. Economic consequences of the COVID-19 outbreak: the need for epidemic preparedness. Front Public Health. 2020;8:241.

11. Nicola M, Alsafi Z, Sohrabi $C$, et al. The socio-economic implications of the coronavirus pandemic (COVID-19): a review. Int J Surg. 2020;78:185-93.

12. Pierce $M$, Hope $H$, Ford $T$, et al. Mental health before and during the COVID19 pandemic: a longitudinal probability sample survey of the UK population. Lancet Psychiatry. 2020;7(10):883-92.

13. Brooks SK, Webster RK, Smith LE, et al. The psychological impact of quarantine and how to reduce it: rapid review of the evidence. Lancet. 2020:395(10227):912-20.

14. Pfefferbaum B, North CS. Mental health and the Covid-19 pandemic. N Engl J Med. 2020;383(6):510-2.

15. Holmes EA, O'Connor RC, Perry VH, et al. Multidisciplinary research priorities for the COVID-19 pandemic: a call for action for mental health science. Lancet Psychiatry. 2020;7(6):547-60.

16. Dubey $S$, Biswas $P$, Ghosh $R$, et al. Psychosocial impact of COVID-19. Diabetes Metab Syndr. 2020;14(5):779-88.
17. Inter-Agency Standing Committee (IASC). Interim briefing note addressing mental health and psycosocial aspects of COVID-19 outbreak (developed by the IASC's Reference Group on Mental Health and Psychosocial Support). 2020. https://interagencystandingcommittee.org/iasc-reference-groupmental-health-and-psychosocial-support-emergency-settings/interimbriefing. Accessed 2 Oct 2020.

18. Lai J, Ma S, Wang Y, et al. Factors associated with mental health outcomes among health care workers exposed to Coronavirus Disease. JAMA Netw Open. 2020;3:e203976

19. Gilan D, Röthke N, Blessin M, et al. Psychomorbidity, resilience, and exacerbating and protective factors during the SARS-CoV-2-pandemic. A systematic literature review and results from the German COSMO-PANEL. Dtsch Arztebl Int. 2020;117(38):625-32.

20. Kisely S, Warren N, McMahon L, Dalais C, Henry I, Siskind D. Occurrence, prevention, and management of the psychological effects of emerging virus outbreaks on healthcare workers: rapid review and meta-analysis. BMJ. 2020;369:m1642.

21. Robertson E, Hershenfield K, Grace SL, Stewart DE. The psychosocial effects of being quarantined following exposure to SARS: a qualitative study of Toronto health care workers. Can J Psychiatr. 2004;49(6):403-7.

22. Vinkers $\mathrm{CH}$, van Amelsvoort T, Bisson Jl, et al. Stress resilience during the coronavirus pandemic. Eur Neuropsychopharmacol. 2020;35:12-6.

23. Yao $\mathrm{H}$, Chen $\mathrm{J}-\mathrm{H}, \mathrm{Xu}$ Y-F. Patients with mental health disorders in the COVID-19 epidemic. Lancet Psychiatry. 2020;7(4):E21.

24. Rogers JP, Chesney E, Oliver D, et al. Psychiatric and neuropsychiatric presentations associated with severe coronavirus infections: a systematic review and meta-analysis with comparison to the COVID-19 pandemic. Lancet Psychiatry. 2020;7(7):611-27.

25. Chan E, Gobat N, Kim JH, et al. Informal home care providers: the forgotten health-care workers during the COVID-19 pandemic. Lancet. 2020; 395(10242):1957-9.

26. Wang H, Li T, Barbarino P, et al. Dementia care during COVID-19. Lancet. 2020;395(10231):1190-1.

27. National Health Service. After-care needs of inpatients recovering from COVID-19. 2020. https://search3.openobjects.com/mediamanager/nottingha mshire/fsd/files/c0388-after-care-needs-of-inpatients-recovering-from-covid-1 9-5-june-2020.pdf. Accessed 2 Oct 2020.

28. Eckardt JP. Caregivers of people with severe mental illness in the COVID-19 pandemic. Lancet Psychiatry. 2020;7(8):e53.

29. da Silva FCT, Neto MLR. Psychological effects caused by the COVID-19 pandemic in health professionals: a systematic review with meta-analysis. Prog Neuro-Psychopharmacol Biol Psychiatry. 2020;104:110062.

30. Xiong J, Lipsitz O, Nasri F, et al. Impact of COVID-19 pandemic on mental health in the general population: a systematic review. J Affect Disord. 2020; 277:55-64.

31. Vindegaard N, Benros ME. COVID-19 pandemic and mental health consequences: systematic review of the current evidence. Brain Behav Immun. 2020;S0889-1591(20):30954-5.

32. Krishnamoorthy Y, Nagarajan R, Saya GK, Menon V. Prevalence of psychological morbidities among general population, healthcare workers and COVID-19 patients amidst the COVID-19 pandemic: a systematic review and meta-analysis. Psychiatry Res. 2020;293:113382.

33. Luo M, Guo L, Yu M, Jiang W, Wang H. The psychological and mental impact of coronavirus disease 2019 (COVID-19) on medical staff and general public - a systematic review and meta-analysis. Psychiatry Res. 2020;291:113190.

34. Preti E, Di Mattei $V$, Perego $G$, et al. The psychological impact of epidemic and pandemic outbreaks on healthcare workers: rapid review of the evidence. Curr Psychiatry Reports. 2020;22(8):43.

35. Cénat JM, Felix N, Blais-Rochette C, et al. Prevalence of mental health problems in populations affected by the Ebola virus disease: a systematic review and meta-analysis. Psychiatry Res. 2020;289:113033.

36. Salari N, Hosseinian-Far A, Jalali R, et al. Prevalence of stress, anxiety, depression among the general population during the COVID-19 pandemic: a systematic review and meta-analysis. Global Health. 2020;16(1):57.

37. Moher D, Liberati A, Tetzlaff J, Altman DG. Preferred reporting items for systematic reviews and meta-analyses: the PRISMA statement. BMJ. 2009;339:b2535.

38. National Heart, Lung, and Blood Institute. Quality Assessment Tool for Observational Cohort and Cross-Sectional Studies 2014. https://www.nhlbi.nih. gov/health-topics/study-quality-assessment-tools. Accessed 2 Oct 2020.

39. Van den Noortgate W, López-López JA, Marín-Martínez F, Sánchez-Meca J. Three-level meta-analysis of dependent effect sizes. Behav Res. 2013:45(2): 576-94. 
40. Higgins JPT, Thompson SG, Spiegelhalter DJ. A re-evaluation of randomeffects meta-analysis. J R Stat Soc Ser A (Statistics in Society). 2009;172(1): 137-59.

41. Qiu J, Shen B, Zhao M, Wang Z, Xie B, Xu Y. A nationwide survey of psychological distress among Chinese people in the COVID-19 epidemic: implications and policy recommendations. Gen Psychiatr. 2020;33(2): e100213.

42. Liu X, Lin $H$, Jiang $H$, et al. Clinical characteristics of hospitalised patients with schizophrenia who were suspected to have coronavirus disease (COVID-19) in Hubei Province, China. Gen Psychiatr. 2020;33(2):e100222.

43. Ahmad AR, Murad HR. The impact of social media on panic during the COVID-19 pandemic in Iraqi Kurdistan: online questionnaire study. J Med Internet Res. 2020;22(5):e19556.

44. Bacon AM, Corr PJ. Coronavirus (COVID-19) in the United Kingdom: a personality-based perspective on concerns and intention to self-isolate. $\mathrm{Br}$ J Health Psychol. 2020;25(4):839-48.

45. Bäuerle A, Teufel M, Musche V, et al. Increased generalized anxiety, depression and distress during the COVID-19 pandemic: a cross-sectional study in Germany. J Public Health (Oxf). 2020;42(4):672-78.

46. Teufel M, Schweda A, Dörrie N, et al. Not all world leaders use twitter in response to the COVID-19 pandemic: impact of the way of Angela Merke on psychological distress, behaviour and risk perception. J Public Health. 2020;42(3):644-6.

47. Buzzi C, Tucci M, Ciprandi R, et al. The psycho-social effects of COVID-19 on Italian adolescents' attitudes and behaviors. Ital J Pediatr. 2020;46(1):69.

48. Cao W, Fang Z, Hou G, et al. The psychological impact of the COVID-19 epidemic on college students in China. Psychiatry Res. 2020;287:112934.

49. Chang J, Yuan Y, Wang D. Mental health status and its influencing factors among college students during the epidemic of COVID-19. Nan Fang Yi Ke Da Xue Xue Bao. 2020;40(2):171-6.

50. Gao J, Zheng P, Jia Y, et al. Mental health problems and social media exposure during COVID-19 outbreak. PLoS One. 2020;15(4):e0231924.

51. Germani A, Buratta L, Delvecchio E, Mazzeschi C. Emerging adults and COVID19: the role of individualism-collectivism on perceived risks and psychological maladjustment. Int J Environ Res Public Health. 2020;17(10):3497.

52. González-Sanguino C, Ausín B, Castellanos MÁ, et al. Mental health consequences during the initial stage of the 2020 coronavirus pandemic (COVID-19) in Spain. Brain Behav Immun. 2020;87:172-6.

53. Harper CA, Satchell LP, Fido D, Latzman RD. Functional fear predicts public health compliance in the COVID-19 pandemic [published online ahead of print, 2020 Apr 27]. Int J Ment Heal Addict. 2020:1-14. https://doi.org/10.1 007/s11469-020-00281-5.

54. Jahanshahi AA, Dinani MM, Madavani AN, Li J, Zhang SX. The distress of Iranian adults during the Covid-19 pandemic - more distressed than the Chinese and with different predictors. Brain Behav Immun. 2020;87:124-5.

55. Lauri Korajlija A. Jokic-Begic N. COVID-19: Concerns and behaviours in Croatia [published online ahead of print, 2020 May 16]. Br J Health Psychol. 2020. https://doi.org/10.1111/bjhp.12425.

56. Lee SA, Mathis AA, Jobe MC, Pappalardo EA. Clinically significant fear and anxiety of COVID-19: a psychometric examination of the coronavirus anxiety scale. Psychiatry Res. 2020;290:113112

57. Lei L, Huang X, Zhang S, Yang J, Yang L, Xu M. Comparison of prevalence and associated factors of anxiety and depression among people affected by versus people unaffected by quarantine during the COVID-19 epidemic in southwestern China. Med Sci Monit. 2020;26:e924609.

58. Li Y, Wang Y, Jiang J, et al. Psychological distress among health professional students during the COVID-19 outbreak [published online ahead of print, 2020 May 11]. Psychol Med. 2020:1-3. https://doi.org/10.1017/S003329172 0001555.

59. Liu N, Zhang F, Wei C, et al. Prevalence and predictors of PTSS during COVID-19 outbreak in China hardest-hit areas: Gender differences matter. Psychiatry Res. 2020;287:112921.

60. Liu S, Liu Y, Liu Y. Somatic symptoms and concern regarding COVID-19 among Chinese college and primary school students: a cross-sectional survey. Psychiatry Res. 2020;289:113070.

61. López J, Perez-Rojo G, Noriega C, et al. Psychological well-being among older adults during the COVID-19 outbreak: a comparative study of the young-old and the old-old adults. Int Psychogeriatr. 2020;32(11):1365-70.

62. Ma K, Zhang Y, Hou T, Wu M, Cai W, Wen T. Investigation of physical and mental health in isolated people during the outbreak of novel coronavirus pneumonia. Chin J Clin Med. 2020;27(1):36-40.
63. Mazza C, Ricci E, Biondi S, et al. A nationwide survey of psychological distress among Italian Ppople during the COVID-19 pandemic: immediate psychological responses and associated factors. Int J Environ Res Public Health. 2020;17(9):3165.

64. McKay D, Yang H, Elhai J, Asmundson GJG. Anxiety regarding contracting COVID-19 related to interoceptive anxiety sensations: the moderating role of disgust propensity and sensitivity. J Anxiety Disord. 2020;73:102233.

65. Moccia $L$, Janiri $D$, Pepe $M$, et al. Affective temperament, attachment style, and the psychological impact of the COVID-19 outbreak: an early report on the Italian general population. Brain Behav Immun. 2020;87:75-9.

66. Odriozola-González P, Planchuelo-Gómez Á, Irurtia MJ, de Luis-García R. Psychological effects of the COVID-19 outbreak and lockdown among students and workers of a Spanish university. Psychiatry Res. 2020;290: 113108.

67. Sanchez TH, Zlotorzynska M, Rai M, Baral SD. Characterizing the impact of COVID-19 on men who have sex with men across the United States in April, 2020. AIDS Behav. 2020;24(7):2024-32.

68. Ozamiz-Etxebarria N, Dosil-Santamaria M, Picaza-Gorrochategui M, IdoiagaMondragon N. Niveles de estrés, ansiedad y depresión en la primera fase del brote del COVID-19 en una muestra recogida en el norte de España [stress, anxiety, and depression levels in the initial stage of the COVID-19 outbreak in a population sample in the northern Spain]. Cad Saude Publica. 2020;36(4):e00054020.

69. Özdin S, Bayrak Öş. Levels and predictors of anxiety, depression and health anxiety during COVID-19 pandemic in Turkish society: the importance of gender. Int J Soc Psychiatry. 2020;66(5):504-11.

70. Pérez-Fuentes MDC, Molero Jurado MDM, Oropesa Ruiz NF, et al. Questionnaire on perception of threat from COVID-19. J Clin Med. 2020;9(4):1196.

71. Ren $Y$, Zhou Y, Qian W, et al. Letter to the editor "a longitudinal study on the mental health of general population during the COVID-19 epidemic in China". Brain Behav Immun. 2020;87:132-3.

72. Reznik A, Gritsenko V, Konstantinov V, Khamenka N, Isralowitz R. COVID-19 fear in Eastern Europe: validation of the fear of COVID-19 scale [published online ahead of print, 2020 May 12]. Int J Ment Health Addict. 2020:1-6. https://doi.org/10.1007/s11469-020-00283-3.

73. Roy D, Tripathy S, Kar SK, Sharma N, Verma SK, Kaushal V. Study of knowledge, attitude, anxiety \& perceived mental healthcare need in Indian population during COVID-19 pandemic. Asian J Psychiatr. 2020;51:102083.

74. Sakib N, Bhuiyan AKMI, Hossain S, et al. Psychometric validation of the Bangla fear of COVID-19 scale: confirmatory factor analysis and Rasch analysis [published online ahead of print, 2020 May 11]. Int J Ment Heal Addict. 2020:1-12. https://doi.org/10.1007/s11469-020-00289-x.

75. Satici B, Gocet-Tekin E, Deniz ME, Satici SA. Adaptation of the fear of COVID19 scale: its association with psychological distress and life satisfaction in Turkey [published online ahead of print, 2020 May 8]. Int J Ment Heal Addict. 2020:1-9. https://doi.org/10.1007/s11469-020-00294-0.

76. Shammi M, Bodrud-Doza M, Towfiqul Islam ARM, Rahman MM. COVID-19 pandemic, socioeconomic crisis and human stress in resource-limited settings: a case from Bangladesh. Heliyon. 2020;6(5):e04063.

77. Shevlin M, Nolan E, Owczarek M, et al. COVID-19-related anxiety predicts somatic symptoms in the UK population. Br J Health Psychol. 2020;25(4):875-82.

78. Soraci P, Ferrari A, Abbiati FA, et al. Validation and psychometric evaluation of the Italian version of the fear of COVID-19 scale [published online ahead of print, 2020 May 4]. Int J Ment Health Addiction. 2020:1-10. https://doi. org/10.1007/s11469-020-00277-1.

79. Tull MT, Edmonds KA, Scamaldo KM, Richmond JR, Rose JP, Gratz KL. Psychological outcomes associated with stay-at-home orders and the perceived impact of COVID-19 on daily life. Psychiatry Res. 2020;289:113098.

80. Tan W, Hao F, Mclntyre RS, et al. Is returning to work during the COVID-19 pandemic stressful? A study on immediate mental health status and psychoneuroimmunity prevention measures of Chinese workforce. Brain Behav Immun. 2020;87:84-92.

81. Tian F, Li H, Tian S, Yang J, Shao J, Tian C. Psychological symptoms of ordinary Chinese citizens based on SCL-90 during the level I emergency response to COVID-19. Psychiatry Res. 2020;288:112992.

82. Tsipropoulou V, Nikopoulou VA, Holeva V, et al. Psychometric properties of the Greek version of FCV-19S [published online ahead of print, 2020 May 26]. Int J Ment Heal Addict. 2020:1-10. https://doi.org/10.1007/s11469-02000319-8.

83. Voitsidis P, Gliatas I, Bairachtari V, et al. Insomnia during the COVID-19 pandemic in a Greek population. Psychiatry Res. 2020;289:113076. 
84. Wang C, Pan R, Wan X, et al. Immediate psychological responses and associated factors during the initial stage of the 2019 Coronavirus disease (COVID-19) epidemic among the general population in China. Int J Environ Res Public Health. 2020;17(5):1729.

85. Wang C, Pan R, Wan X, et al. A longitudinal study on the mental health of general population during the COVID-19 epidemic in China. Brain Behav Immun. 2020;87:40-8.

86. Wang $\mathrm{H}$, Xia Q, Xiong Z, et al. The psychological distress and coping styles in the early stages of the 2019 coronavirus disease (COVID-19) epidemic in the general mainland Chinese population: a web-based survey. PLoS One. 2020;15(5):e0233410

87. Wang Y, Di Y, Ye J, Wei W. Study on the public psychological states and its related factors during the outbreak of coronavirus disease 2019 (COVID-19) in some regions of China. Psychol Health Med. 2021;26(1):13-22.

88. Yang $\mathrm{H}, \mathrm{Ma}$ J. How an epidemic outbreak impacts happiness: factors that worsen (vs. protect) emotional well-being during the coronavirus pandemic. Psychiatry Res. 2020;289:113045.

89. Yuan $\mathrm{R}, \mathrm{Xu} \mathrm{Q}-\mathrm{H}, \mathrm{Xia}$ C-C, et al. Psychological status of parents of hospitalized children during the COVID-19 epidemic in China. Psychiatry Res. 2020;288:112953.

90. Zhang SX, Huang H, Wei F. Geographical distance to the epicenter of Covid-19 predicts the burnout of the working population: ripple effect or typhoon eye effect? Psychiatry Res. 2020;288:112998.

91. Zhang SX, Wang Y, Rauch A, Wei F. Unprecedented disruption of lives and work: health, distress and life satisfaction of working adults in China one month into the COVID-19 outbreak. Psychiatry Res. 2020;288:112958.

92. Zhang Y, Ma ZF. Impact of the COVID-19 pandemic on mental health and quality of life among local residents in Liaoning Province, China: a crosssectional study. Int J Environ Res Public Health. 2020;17(7):2381.

93. Zhou SJ, Zhang LG, Wang LL, et al. Prevalence and socio-demographic correlates of psychological health problems in Chinese adolescents during the outbreak of COVID-19. Eur Child Adolesc Psychiatry. 2020;29(6):749-58.

94. Abdessater $M$, Rouprêt $M$, Misrai $V$, et al. COVID19 pandemic impacts on anxiety of French urologist in training: outcomes from a national survey. Prog Urol. 2020;30(8-9):448-55

95. Ahmed MA, Jouhar $\mathrm{R}$, Ahmed N, et al. Fear and practice modifications among dentists to combat novel coronavirus disease (COVID-19) outbreak Int J Environ Res Public Health. 2020;17(8):2821.

96. Alhaj AK, Al-Saadi T, Mohammad F, Alabri S. Neurosurgery residents' perspective on COVID-19: knowledge, readiness, and impact of this pandemic. World Neurosurg. 2020;139:e848-58.

97. Amerio A, Bianchi D, Santi F, et al. Covid-19 pandemic impact on mental health: a web-based cross-sectional survey on a sample of Italian general practitioners. Acta Biomed. 2020;91(2):83-8.

98. Badahdah AM, Khamis F, Mahyijari NA. The psychological well-being of physicians during COVID-19 outbreak in Oman. Psychiatry Res. 2020;289: 113053

99. Bohlken J, Schömig F, Seehagen T, et al. Erfahrungen und Belastungserleben niedergelassener Neurologen und Psychiater während der COVID-19Pandemie [experience of practice-based psychiatrists and neurologists during the COVID-19 pandemic]. Psychiatr Prax. 2020;47(4):214-7.

100. Cai H, Tu B, Ma J, et al. Psychological impact and coping strategies of frontline medical staff in hunan between January and March 2020 during the outbreak of coronavirus disease 2019 (COVID-19) in Hubei, China. Med Sci Monit. 2020;26:e924171.

101. Cai W, Lian B, Song X, Hou T, Deng G, Li H. A cross-sectional study on mental health among health care workers during the outbreak of Corona virus disease 2019. Asian J Psychiatr. 2020;51:102111.

102. Chew NWS, Lee GKH, Tan BYQ, et al. A multinational, multicentre study on the psychological outcomes and associated physical symptoms amongst healthcare workers during COVID-19 outbreak. Brain Behav Immun. 2020;88:559-65.

103. Consolo U, Bellini P, Bencivenni D, lani C, Checchi V. Epidemiological aspects and psychological reactions to COVID-19 of dental practitioners in the northern Italy districts of Modena and Reggio Emilia. Int J Environ Res Public Health. 2020;17(10):3459.

104. Gan X, Shi Z, Chair SY, Cao X, Wang Q. Willingness of Chinese nurses to practice in Hubei combating the coronavirus disease 2019 epidemic: a cross-sectional study. J Adv Nurs. 2020;76(8):2137-50.

105. Huang JZ, Han MF, Luo TD, Ren AK, Zhou XP. Mental health survey of medical staff in a tertiary infectious disease hospital for COVID-19. Zhonghua Lao Dong Wei Sheng Zhi Ye Bing Za Zhi. 2020;38(3):192-5.
106. Kang L, Ma S, Chen M, et al. Impact on mental health and perceptions of psychological care among medical and nursing staff in Wuhan during the 2019 novel coronavirus disease outbreak: a cross-sectional study. Brain Behav Immun. 2020;87:11-7.

107. Khusid JA, Weinstein CS, Becerra AZ, et al. Well-being and education of urology residents during the COVID-19 pandemic: results of an American National Survey. Int J Clin Pract. 2020;74(9):e13559.

108. Mo $Y$, Deng $L$, Zhang $L$, et al. Work stress among Chinese nurses to support Wuhan in fighting against COVID-19 epidemic. J Nurs Manag. 2020;28(5):1002-9.

109. Pu J, Li G, Cao L, Wu Y, Xu L. Investigation and analysis of the psychological status of the clinical nurses in a class. A hospital facing the novel coronavirus pneumonia. Chongqing Med. 2020;36:E015.

110. Rossi R, Socci V, Pacitti F, et al. Mental health outcomes among frontline and second-line health care workers during the coronavirus disease 2019 (COVID-19) pandemic in Italy. JAMA Netw Open. 2020;3(5):e2010185.

111. Sahu D, Agrawal T, Rathod V, Bagaria V. Impact of COVID 19 lockdown on orthopaedic surgeons in India: a survey. J Clin Orthop Trauma. 2020; 11(Suppl 3):S283-90

112. Shacham M, Hamama-Raz Y, Kolerman R, Mijiritsky O, Ben-Ezra M, Mijiritsky E. COVID-19 factors and psychological factors associated with elevated psychological distress among dentists and dental hygienists in Israel. Int J Environ Res Public Health. 2020;17(8):2900.

113. Suleiman A, Bsisu I, Guzu H, et al. Preparedness of frontline doctors in Jordan healthcare facilities to COVID-19 outbreak. Int J Environ Res Public Health. 2020;17(9):3181.

114. Tan BYQ, Chew NWS, Lee GKH, et al. Psychological impact of the COVID-19 pandemic on health care workers in Singapore. Ann Intern Med. 2020; 173(4):317-20

115. Wang S, Xie L, Xu Y, Yu S, Yao B, Xiang D. Sleep disturbances among medical workers during the outbreak of COVID-2019. Occup Med. 2020; 70(5):364-9.

116. Wu K, Wei X. Analysis of psychological and sleep status and exercise rehabilitation of front-line clinical staff in the fight against COVID-19 in China. Med Sci Monit Basic Res. 2020;26:e924085.

117. Xiao H, Zhang Y, Kong D, Li S, Yang N. The effects of social support on sleep quality of medical staff treating patients with coronavirus disease 2019 (COVID-19) in January and February 2020 in China. Med Sci Monit. 2020;26:e923549.

118. Xu J, Xu Q-H, Wang C-M, Wang J. Psychological status of surgical staff during the COVID-19 outbreak. Psychiatry Res. 2020;288:112955.

119. Yin Q, Sun Z, Liu T, et al. Posttraumatic stress symptoms of health care workers during the corona virus disease 2019. Clin Psychol Psychother. 2020;27(3):384-95

120. Zhang C, Yang L, Liu S, et al. Survey of insomnia and related social psychological factors among medical staff involved in the 2019 novel coronavirus disease outbreak. Front Psychiatry. 2020;11:306.

121. Zhang SX, Liu J, Afshar Jahanshahi A, et al. At the height of the storm: healthcare staff's health conditions and job satisfaction and their associated predictors during the epidemic peak of COVID-19. Brain Behav Immun. 2020;87:144-6.

122. Zhu J, Sun $L$, Zhang $L$, et al. Prevalence and influencing factors of anxiety and depression symptoms in the first-line medical staff fighting against COVID-19 in Gansu. Front Psychiatry. 2020;11:386.

123. Cai X, Hu X, Ekumi IO, et al. Psychological distress and its correlates among COVID-19 survivors during early convalescence across age groups. Am J Geriatr Psychiatry. 2020;28(10):1030-9.

124. Yuan B, Li W, Liu H, et al. Correlation between immune response and selfreported depression during convalescence from COVID-19. Brain Behav Immun. 2020;88:39-43.

125. Durankuş F, Aksu E. Effects of the COVID-19 pandemic on anxiety and depressive symptoms in pregnant women: a preliminary study [published online ahead of print, 2020 May 18]. J Matern Fetal Neonatal Med. 2020:1-7. https://doi.org/10.1080/14767058.2020.1763946.

126. Li X, Dai T, Wang H, et al. Clinical analysis of suspected COVID-19 patients with anxiety and depression. Zhejiang Da Xue Xue Bao Yi Xue Ban. 2020; 49(2):203-8

127. Wu Y, Zhang C, Liu H, et al. Perinatal depressive and anxiety symptoms of pregnant women during the coronavirus disease 2019 outbreak in China. Am J Obstet Gynecol. 2020;223(2):240.e1-9.

128. Xu H, Yang $K$, Yang $G$, et al. Explore the optimal resolvent of medical needs and mental health for patients with lung cancer during epidemic novel coronavirus pneumonia. Zhongguo Fei Ai Za Zhi. 2020;23(4):247-54. 
129. Yassa M, Birol P, Yirmibes C, et al. Near-term pregnant women's attitude toward, concern about and knowledge of the COVID-19 pandemic. J Matern Fetal Neonatal Med. 2020;33(22):3827-34.

130. Büntzel J, Klein M, Keinki C, Walter S, Büntzel J, Hübner J. Oncology services in corona times: a flash interview among German cancer patients and their physicians. J Cancer Res Clin Oncol. 2020;146(10):2713-5.

131. Guo Q, Zheng Y, Shi J, et al. Immediate psychological distress in quarantined patients with COVID-19 and its association with peripheral inflammation: a mixed-method study. Brain Behav Immun. 2020;88:17-27.

132. Hao F, Tan $W$, Jiang $L$, et al. Do psychiatric patients experience more psychiatric symptoms during COVID-19 pandemic and lockdown? A casecontrol study with service and research implications for immunopsychiatry. Brain Behav Immun. 2020;87:100-6.

133. Hao X, Zhou D, Li Z, et al. Severe psychological distress among patients with epilepsy during the COVID-19 outbreak in Southwest China. Epilepsia. 2020;61(6):11661173.

134. Huang Y, Zhao N. Mental health burden for the public affected by the COVID-19 outbreak in China: Who will be the high-risk group? [retracted in: Psychol Health Med. 2020 Oct 23::1]. Psychol Health Med. 2021;26(1):23-34.

135. lasevoli F, Fornaro M, D'Urso G, et al. Psychological distress in patients with serious mental illness during the COVID-19 outbreak and one-month mass quarantine in Italy [published online ahead of print, 2020 May 19]. Psychol Med. 2020;1-3. https://doi.org/10.1017/S0033291720001841.

136. Jin $\mathrm{YH}$, Huang $\mathrm{Q}$, Wang $\mathrm{YY}$, et al. Perceived infection transmission routes, infection control practices, psychosocial changes, and management of COVID-19 infected healthcare workers in a tertiary acute care hospital in Wuhan: a cross-sectional survey. Mil Med Res. 2020;7(1):24.

137. Ko N-Y, Lu W-H, Chen Y-L, et al. COVID-19-related information sources and psychological well-being: an online survey study in Taiwan. Brain Behav Immun. 2020;87:153-4

138. Li Z, Ge J, Yang M, et al. Vicarious traumatization in the general public, members, and non-members of medical teams aiding in COVID-19 control. Brain Behav Immun. 2020;88:916-9.

139. Lu W, Wang H, Lin Y, Li L. Psychological status of medical workforce during the COVID-19 pandemic: a cross-sectional study. Psychiatry Res. 2020;288: 112936

140. Ni MY, Yang L, Leung CMC, et al. Mental health, risk factors, and social media use during the COVID-19 epidemic and cordon sanitaire among the community and health professionals in Wuhan, China: cross-sectional survey. JMIR Mental Health. 2020;7(5):e19009.

141. Wu W, Zhang $Y$, Wang $P$, et al. Psychological stress of medical staffs during outbreak of COVID-19 and adjustment strategy. J Med Virol. 2020;92(10): 1962-70. https://doi.org/10.1002/jmv.25914.

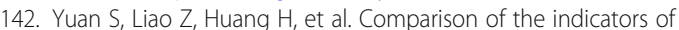
psychological stress in the population of Hubei province and non-endemic provinces in China during two weeks during the coronavirus disease 2019 (COVID-19) outbreak in February 2020. Med Sci Monit. 2020;26:e923767.

143. Zhang J, Lu H, Zeng $H$, et al. The differential psychological distress of populations affected by the COVID-19 pandemic. Brain Behav Immun. 2020; 87:49-50.

144. Zhang WR, Wang K, Yin L, et al. Mental health and psychosocial problems of medical health workers during the COVID-19 epidemic in China. Psychother Psychosom. 2020;89(4):242-50.

145. Zhu S, Wu Y, Zhu C-Y, et al. The immediate mental health impacts of the COVID-19 pandemic among people with or without quarantine managements. Brain Behav Immun. 2020;87:56-8.

146. Olagoke AA, Olagoke $O O$, Hughes AM. Exposure to coronavirus news on mainstream media: the role of risk perceptions and depression. $\mathrm{Br} \mathrm{J}$ Health Psychol. 2020;25(4):865-74.

147. Sutin AR, Robinson E, Daly M, et al. BMI, Weight discrimination, and psychological, behavioral, and interpersonal responses to the coronavirus pandemic. Obesity (Silver Spring, Md). 2020;28(9):1590-4.

148. Alosaimi FD, Alawad HS, Alamri AK, et al. Patterns and determinants of stress among consultant physicians working in Saudi Arabia. Adv Med Educ Pract. 2018;9:165-74

149. Balestrieri M, Isola M, Quartaroli M, Roncolato M, Bellantuono C. Assessing mixed anxiety-depressive disorder. A national primary care survey. Psychiatry Res. 2010;176(2-3):197-201.

150. Basta M, Karakonstantis S, Koutra K, et al. NEET status among young Greeks: association with mental health and substance use. J Affect Disord. 2019;253:210-7.
151. Bilgel N, Bayram N. Turkish version of the depression anxiety stress scale (DASS-42): psychometric properties. Nöropsikiyatri Arşivi. 2010;47(2):118-26.

152. Bonfiglio NS, Renati R, Hjemdal O, Friborg O. The resilience scale for adults in Italy: a validation study comparing clinical substance abusers with a nonclinical sample. Psychol Addict Behav. 2016;30(4):509-15.

153. Bottesi G, Ghisi M, Altoè G, Conforti $E$, Melli G, Sica $C$. The Italian version of the Depression Anxiety Stress Scales-21: Factor structure and psychometric properties on community and clinical samples. Compr Psychiatry. 2015;60: 170-81.

154. Cai S, Lin H, Hu X, Cai YX, Chen K, Cai WZ. High fatigue and its associations with health and work related factors among female medical personnel at 54 hospitals in Zhuhai, China. Psychol Health Med. 2017;23(3):304-16.

155. Carlucci L, Watkins MW, Sergi MR, Cataldi F, Saggino A, Balsamo M. Dimensions of anxiety, age, and gender: assessing dimensionality and measurement invariance of the State-Trait for Cognitive and Somatic Anxiety (STICSA) in an Italian sample. Front Psychol. 2018;9:2345.

156. Carta MG, Angermeyer MC, Matschinger H, Holzinger A, Floris F, Moro MF. Perception of depressive symptoms by the Sardinian public: results of a population study. BMC Psychiatry. 2013;13:57.

157. Choueiry N, Salamoun T, Jabbour H, El Osta N, Hajj A, Rabbaa Khabbaz L. Insomnia and relationship with anxiety in university students: a crosssectional designed study. PLoS One. 2016;11(2):e0149643.

158. Chung KF, Tso KC. Relationship between insomnia and pain in major depressive disorder: a sleep diary and actigraphy study. Sleep Med. 2010; $11(8): 752-8$.

159. Dadfar M, Salabifard S, Dadfar T, Roudbari M, Safarabad NM. Validation of the Patient Health Questionnaire-2 with Iranian students. Ment Health Relig Cult. 2019;22(10):1048-56.

160. Dong H, Zhang Q, Sun Z, Sang F, Xu Y, et al. BMC Psychiatry. 2017;17(1):241.

161. Ertekin Pinar S, Duran Aksoy O, Daglar G, Yurtsal ZB, Cesur B. Effect of stress management training on depression, stress and coping strategies in pregnant women: a randomised controlled trial. J Psychosom Obstet Gynaecol. 2017;39(3):203-10.

162. Fleishman JA, Zuvekas SH. Global self-rated mental health: associations with other mental health measures and with role functioning. Med Care. 2007; 45(7):602-9.

163. García-Campayo J, Zamorano E, Ruiz MA, Pérez-Páramo M, López-Gómez V, Rejas J. The assessment of generalized anxiety disorder: psychometric validation of the Spanish version of the self-administered GAD-2 scale in daily medical practice. Health Qual Life Outcomes. 2012;10:114.

164. Ho RT, Fong TC, Wan AH, et al. A randomized controlled trial on the psychophysiological effects of physical exercise and tai-chi in patients with chronic schizophrenia. Schizophr Res. 2016;171(1-3):42-9.

165. Hossain S, Anjum A, Uddin ME, Rahman MA, Hossain MF. Impacts of sociocultural environment and lifestyle factors on the psychological health of university students in Bangladesh: a longitudinal study. J Affect Disord. 2019:256:393-403

166. Huang F, Wang H, Wang Z, et al. Psychometric properties of the perceived stress scale in a community sample of Chinese. BMC Psychiatry. 2020;20(1): 130.

167. Ivziku D, Clari M, Piredda M, De Marinis MG, Matarese M. Anxiety, depression and quality of life in chronic obstructive pulmonary disease patients and caregivers: an actor-partner interdependence model analysis. Qual Life Res. 2018;28(2):461-72.

168. Jeyagurunathan A, Sagayadevan V, Abdin E, et al. Psychological status and quality of life among primary caregivers of individuals with mental illness: a hospital based study. Health Qual Life Outcomes. 2017;15(1):106.

169. Jin $H, W u$ W, Zhang M. Preliminary analysis of SCL-90 assessment results of Chinese normal people. Chinese J Nerv Ment Dis. 1986;12:260-3.

170. Lee KS, Yoo TK, Liao L, et al. Association of lower urinary tract symptoms and $O A B$ severity with quality of life and mental health in China, Taiwan and South Korea: results from a cross-sectional, population-based study. BMC Urol. 2017;17(1):108

171. Lin R-M, Xie S-S, Yan W-J, Yan Y-W. Factor structure and psychometric properties of the insomnia severity index in mainland China. Soc Behav Personal Int J. 2018;46(2):209-18.

172. Liu H, Zhang $H$, Xiao W, et al. Scales for evaluating depressive symptoms in Chinese patients with schizophrenia. J Nerv Ment Dis. 2009:197(2):140-2.

173. Liu RQ, Qian Z, Trevathan E, et al. Poor sleep quality associated with high risk of hypertension and elevated blood pressure in China: results from a large population-based study. Hypertens Res. 2016;39(1):54-9. 
174. Liu X, Wang XQ, Zhang WN, Bo QY, Zhu SY. Study on the relationship between quality of nursing professional life and depression tendency of nursing staff. Zhonghua Lao Dong Wei Sheng Zhi Ye Bing Za Zhi. 2020; 38(5):365-8

175. Löwe B, Decker $\mathrm{O}$, Müller $\mathrm{S}$, et al. Validation and standardization of the generalized anxiety disorder screener (GAD-7) in the general population. Med Care. 2008;46(3):266-74.

176. Löwe B, Wahl I, Rose M, et al. A 4-item measure of depression and anxiety: validation and standardization of the patient health Questionnaire-4 (PHQ-4) in the general population. J Affect Disord. 2010;122(1-2):86-95.

177. Lu S, Hu S, Guan Y, et al. Measurement invariance of the Depression Anxiety Stress Scales-21 across gender in a sample of Chinese university students. Front Psychol. 2018;9:2064.

178. Lu W, Bian Q, Wang W, Wu X, Wang Z, Zhao M. Chinese version of the Perceived Stress Scale-10: a psychometric study in Chinese university students. PLoS One. 2017;12(12):e0189543.

179. Paparrigopoulos T, Tzavara C, Theleritis C, Psarros C, Soldatos C, Tountas Y. Insomnia and its correlates in a representative sample of the Greek population. BMC Public Health. 2010;10:531.

180. Pereira-Lima K, Loureiro SR. Burnout, anxiety, depression, and social skills in medical residents. Psychol Health Med. 2014;20(3):353-62

181. Ramón-Arbués E, Martínez Abadía B, Granada López JM, et al. Conducta alimentaria y su relación con el estrés, la ansiedad, la depresión y el insomnio en estudiantes universitarios [Eating behavior and relationships with stress, anxiety, depression and insomnia in university students.]. Nutr Hosp. 2019;36(6):1339-45.

182. Sasaki N, Imamura K, Thuy TाT, et al. Validation of the Job Content Questionnaire among hospital nurses in Vietnam. J Occup Health. 2019; 62(1):e12086.

183. Schmidt RE, Cullati S, Mostofsky E, et al. Healthcare-related regret among nurses and physicians is associated with self-rated insomnia severity: a cross-sectional study. PLoS One. 2015;10(10):e0139770.

184. Sinclair SJ, Siefert CJ, Slavin-Mulford JM, Stein MB, Renna M, Blais MA. Psychometric evaluation and normative data for the depression, anxiety, and stress scales-21 (DASS-21) in a nonclinical sample of U.S. adults. Eval Health Prof. 2012;35(3):259-79.

185. Wang K, Shi HS, Geng FL, et al. Cross-cultural validation of the depression anxiety stress Scale-21 in China. Psychol Assess. 2016;28(5):e88-100.

186. Wang W, Bian Q, Zhao Y, et al. Reliability and validity of the Chinese version of the patient health questionnaire (PHQ-9) in the general population. Gen Hosp Psychiatry. 2014;36(5):539-44.

187. Wang WL, Zhou YQ, Chai NN, Li GH, et al. Sleep disturbance and quality of life in clinically stable inpatients with schizophrenia in rural China. Qual Life Res. 2020;29(10):2759-68.

188. Wang X, Wang X, Ma H. Handbook of Mental Health Assessment Scale. Chin Ment Health J. 1999;13(1):31-5.

189. Wang $Y$, Yang $X$, Song $X$, et al. Co-treatment of buspirone with atypical antipsychotic drugs (AAPDs) improved neurocognitive function in chronic schizophrenia. Schizophr Res. 2019;209:135-40.

190. Yang $X$, Zhang $P$, Zhao J, et al. Confucian culture still matters: the benefits of Zhongyong thinking (doctrine of the mean) for mental health. J CrossCult Psychol. 2016;47(8):1097-113.

191. Yıldırım A, Boysan M, Kefeli MC. Psychometric properties of the Turkish version of the depression anxiety stress Scale-21 (DASS-21). Br J Guid Couns. 2018:46(5):582-95.

192. Yu B, Gu Y, Bao X, et al. Distinct associations of computer/mobile devices use and TV watching with depressive symptoms in adults: a large population study in China. Depress Anxiety. 2019;36(9):879-86.

193. Zhou J, Yang Y, Qiu X, et al. Relationship between anxiety and burnout among Chinese physicians: a moderated mediation model. PLoS One. 2016; 11(8):e0157013.

194. Kunzler AM, Helmreich I, Chmitorz A, et al. Psychological interventions to foster resilience in healthcare professionals. Cochrane Database Syst Rev. 2020;7:CD012527.

195. Salazar de Pablo G, Vaquerizo-Serrano J, Catalan A, et al. Impact of coronavirus syndromes on physical and mental health of health care workers: systematic review and meta-analysis. J Affect Disord. 2020;275:48-57.

196. Bonanno GA, Mancini AD. Beyond resilience and PTSD: mapping the heterogeneity of responses to potential trauma. Psychol Trauma Theory Res Pract Policy. 2012:4:74-83.
197. Mancini AD. Heterogeneous mental health consequences of COVID-19: costs and benefits. Psychol Trauma Theory Res Pract Policy. 2020;12(S1):S15-6.

198. Ahrens KF, Neumann RJ, Kollmann B, et al. Differential impact of COVIDrelated lockdown on mental health in Germany. World Psychiatry. 2021;20:1.

199. Veer IM, Riepenhausen A, Zerban M, et al. Psycho-social factors associated with mental resilience in the Corona lockdown. 2020. https://psyarxiv.com/4 z62t/. Accessed 4 Nov 2020.

200. Löwe B, Kroenke K, Herzog W, Gräfe K. Measuring depression outcome with a brief self-report instrument: sensitivity to change of the patient health questionnaire (PHQ-9). J Affect Disord. 2004;81(1):61-6.

201. Duffy FF, Chung H, Trivedi M, Rae DS, Regier DA, Katzelnick DJ. Systematic use of patient-rated depression severity monitoring: is it helpful and feasible in clinical psychiatry? Psychiatr Serv. 2008;59(10):1148-54.

202. Erskine HE, Moffitt TE, Copeland WE, et al. A heavy burden on young minds: the global burden of mental and substance use disorders in children and youth. Psychol Med. 2015;45(7):1551-63.

203. IntHout J, loannidis JPA, Rovers MM, Goeman JJ. Plea for routinely presenting prediction intervals in meta-analysis. BMJ Open. 2016;6(7): e010247.

\section{Publisher's Note}

Springer Nature remains neutral with regard to jurisdictional claims in published maps and institutional affiliations.
Ready to submit your research? Choose BMC and benefit from:

- fast, convenient online submission

- thorough peer review by experienced researchers in your field

- rapid publication on acceptance

- support for research data, including large and complex data types

- gold Open Access which fosters wider collaboration and increased citations

- maximum visibility for your research: over $100 \mathrm{M}$ website views per year

At BMC, research is always in progress.

Learn more biomedcentral.com/submissions 